\title{
Adaptive Control of Nonlinear Systems Using Spline Function Approximation Techniques
}

\author{
A Dissertation \\ Presented to \\ the Faculty of the School of Engineering and Applied Science \\ University of Virginia \\ In Partial Fulfillment \\ of the Requirements for the Degree \\ Doctor of Philosophy \\ Electrical Engineering
}

by

Hesham M. Shageer

May 2013 


\section{APPROVAL SHEET}

This dissertation is submitted in partial fulfillment of the requirements for the degree of

Doctor of Philosophy in Electrical Engineering

Hesham M. Shageer, Author

This dissertation has been read and approved by the examining committee:

Prof. Gang Tao, Dissertation Advisor

Prof. Zongli Lin, Committee Chairman

Prof. Stephen G. Wilson, Electrical and Computer Engineering

Prof. Carl R. Knospe, Mechanical and Aerospace Engineering

Prof. Harry Powell, Electrical and Computer Engineering

Accepted for the School of Engineering and Applied Science:

Dean, School of Engineering and Applied Science

May 2013 


\section{Dedication and Acknowledgements}

I dedicate this $\mathrm{PhD}$ Dissertation to my parents and loved ones who have been encouraging me and supporting my quest for beneficial knowledge every step of the way. I would also like to begin by expressing my extreme gratitude and sincere thanks to my advisor, Prof. Gang Tao, for his guidance and support that has been consistent throughout my many years at the University, even dating as far back as my undergraduate days. His ability to spread knowledge and achieve perfect dedication are very inspiring, and I am grateful to have been privileged enough to be one of his students and to have learned from him.

I am honored to thank my PhD Dissertation advisory committee members - Prof. Zongli Lin, Prof. Carl Knospe, Prof. Harry Powell, and Prof. Steve Wilson - for their flexible time schedule, valuable research suggestions, agreement to serve on my committee and eye opening technical questions.

I would like to acknowledge the Saudi Arabian Cultural Mission (SACM) and the UVA Financial Aid Office for their consistent financial support throughout my education. My deep appreciation also goes out to my friends and colleagues Yu Liu, Jason Burkholder, Dipankar Deb, Qian Sang, Azmee Rahman, Ala Abdelbakhi, Jimmy Johnson and Charu Singhal for their intellectual discussion and lifetime of help. Finally, I am pleased to acknowledge the financial support provided by NASA through Barron Associates Inc.

Hesham M. Shageer

University of Virginia 


\section{Abstract}

Adaptive control theory provides an effective tool to overcome the modeling uncertainties associated with the design of dynamical control systems. Real life dynamical systems contain parametric, structural, modeling and environmental uncertainties that might be difficult to formulate into an accurate model or be partially unknown. Therefore, some of these system uncertainties can be taken into consideration, and especially the modeling error effects due to inaccuracies or over simplifications which constitute a major design challenge that may limit the level of achievable performance.

Better modeling and control techniques can be developed by employing an approximation based spline function network to adaptively control, approximate the uncertain system nonlinearities and estimate the unknown model parameters online. A number of theoretical and computational challenges must be overcome to reach a significant contribution in this field of approximation based adaptive control theory. These challenges include: reduction of modeling error effects on control system performance, computation of a large number of adaptable parameters that might be necessary to achieve desired system performance, ability to ensure convergence of the system output, state, and approximator parameters for tracking objectives, and development of new approximation structures that can adapt or adjust to necessary structural changes to ensure the best available fit during online operation.

In this dissertation we address these challenges by developing advanced approximation based adaptive control techniques using spline functions for nonlinear systems, along with substantial results to solve some control problems of urgent relevance to aircraft and spacecraft applications. Specifically, a spline function approximation 
based adaptive control scheme is designed and analyzed for two complex examples: a commercial aircraft with actuator nonlinearities due to synthetic jet inputs and a nonlinear spacecraft model with unknown liquid fuel slosh model parameters. The designed controller structures employ a multivariable spline function network to approximate the uncertain system nonlinearities and estimate the unknown model parameters online. They guarantee closed-loop stability and convergence of the tracking error to a small residual set. Parameter projection-based adaptive laws are employed to ensure desired closed-loop stability, approximator parameter convergence, and desired tracking properties are maintained throughout the entire operating envelope. Simulation results are presented to illustrate how the desired system performance is achieved and maintained for a realistic set of operating conditions. This research offers the extension of our preliminary results to include the development of an approximation based adaptive control design and analysis study for more complex nonlinear dynamical systems that suffer from similar drawbacks and limitations. 


\section{Contents}

1 Introduction and Overview 1

1.1 Approximation Based Control Methodology . . . . . . . . . . . . 1

1.2 Literature Overview . . . . . . . . . . . . . . . 6

1.2.1 Aircraft Control with Synthetic Jet Actuators . . . . . . . . . 6

1.2.2 Modeling and Control of Spacecraft with Fuel Slosh . . . . . . 8

1.3 Contributions Summary . . . . . . . . . . . . . . . . . . . 10

1.4 Dissertation Preview . . . . . . . . . . . . . . . . 12

2 Technical Background and Problem Formulation 13

2.1 Splines for Approximation and Control . . . . . . . . . . . . 13

2.2 Approximation Based Adaptive Control Schemes . . . . . . . . . . . 20

2.3 Problem Formulation . . . . . . . . . . . . . . . . . . 25

3 Modeling of Spacecraft with Fuel Slosh Dynamics 31

3.1 A Spacecraft Model with Fuel Slosh . . . . . . . . . . . . . . . . 31

3.2 MIMO Spacecraft with Fuel Slosh . . . . . . . . . . . . . . 38

3.3 Multi-Mass Fuel Slosh Dynamics . . . . . . . . . . . . . . 50

4 Synthetic Jets for Aircraft 53

4.1 Airflow Shaping and Control . . . . . . . . . . . . 54

4.2 Mathematical Model . . . . . . . . . . . . . . . . . . 57

4.3 Actuator Nonlinearity Characteristic . . . . . . . . . . . 59 
4.4 Other Synthetic Jet Actuator Applications . . . . . . . . . . . . . 61

5 Approximation Based Adaptive Control of Spacecraft with Fuel Slosh using Spline Functions $\quad 62$

5.1 Approximated SISO System Model . . . . . . . . . . . . 62

5.1.1 Spline Function Approximation Technique . . . . . . . . . . . 64

5.1 .2 Stability Analysis . . . . . . . . . . . . . . 67

5.1 .3 Simulations ................... 70

5.2 Approximation and Control of MIMO Model . . . . . . . . . . . . 74

5.2.1 Splines for Approximation and Control . . . . . . . . . . . 81

5.2.2 Space Flight Control Performance Evaluation . . . . . . . . . 87

6 Adaptive Compensation of Synthetic Jet Actuator Nonlinearities 91

6.1 Control Error . . . . . . . . . . . . . . . . . . . 94

6.2 Adaptive Feedback Control System . . . . . . . . . . . . . . . . 96

6.2.1 State Feedback Control . . . . . . . . . . . . . . 97

6.2.2 Adaptive Laws . . . . . . . . . . . . . . . . . . . . 98

6.3 Aircraft Flight Control Performance . . . . . . . . . . . . . . . 103

6.3.1 Linear Aircraft Dynamic Model . . . . . . . . . . . . . . . 103

6.3.2 Linear Feedback Law . . . . . . . . . . . . . . . . . . . . 104

6.3.3 Simulation ..................... 105

7 Conclusions and Outlook $\quad 111$

7.1 Conclusions . . . . . . . . . . . . . . . . 111

7.2 Technology Outlook . . . . . . . . . . . . . . . . . 112 


\section{List of Figures}

1.1 Closed-loop system output tracking performance for the control law (1.2) applied to the system equation in (1.1) with no function approximator $\hat{f}(x)=0 . \ldots \ldots \ldots \ldots$. . . . . . . . . . . . . 4

1.2 Output tracking performance for (1.2) applied to (1.1) with function approximation $\hat{f}(x)=0.7 \cos x$, the solid line is the desired reference trajectory $y_{d}(t)$ and the dashed line is the system output response $y(t)$. The tracking error is plotted on the bottom part of the figure. . . . .

2.1 Cubic B-spline basis elements for $(2.2) \ldots \ldots \ldots$

2.2 The solid line in the top plot shows the desired function $f(x)=\cos 2 x$ and the dashed line is the spline function approximator $\hat{f}(x, \theta)$. The basis functions $\phi_{1}, \ldots, \phi_{8}$ are plotted on the bottom part of the figure. 18

2.3 Multivariate cubic B-spline basis elements. . . . . . . . . . . . . . . . 19

2.4 The solid line in the top plot shows the desired output $y_{d}(t)=\cos t$ and the dashed line is the actual output response $y(t)$ with the spline function approximator $\hat{f}(x ; \theta)$. The tracking error $e(t)$ is plotted on the bottom part of the figure. . . . . . . . . . . . . . . . 19

2.5 Basic model reference direct adaptive feedback control. . . . . . . . . 21

2.6 Indirect adaptive control system. . . . . . . . . . . . . . . . 22

2.7 Basic adaptive state feedback inverse compensation control scheme. . 26

3.1 Spacecraft with fuel slosh dynamics [9]. . . . . . . . . . . . . . 32 
3.2 Free-body diagram for fuel slosh as pendulum approximation. . . . . 33

3.3 Free-body diagram for spacecraft. . . . . . . . . . . . . 33

3.4 Phase portrait $x_{3}-x_{4}$ for the zero dynamics. . . . . . . . . . 38

3.5 Spacecraft with fuel slosh dynamics [51]. . . . . . . . . . . . . . . 39

3.6 Phase portrait $x_{6}-x_{5}$ for the zero dynamics system (3.37)-(3.39). . . 47

3.7 A multi-slosh mode pendulum model for a spacecraft with a gimbaled thruster with multiple inputs. [52]. . . . . . . . . . . . . . . 50

4.1 Physical structure of a synthetic jet actuator. . . . . . . . . 53

4.2 Clean airfoil around an aircraft wing. . . . . . . . . . . . . . . 54

4.3 Pressure bubble disturbing surface and airfoil around an aircraft wing. 55

4.4 Separation of airflow resulting in stall. . . . . . . . . . . . 56

4.5 Reattached airflow with synthetic jets at the leading edge. . . . . . . 56

4.6 Nonlinear profile $f(v, \alpha)$ for varying $\alpha$ and $v \ldots \ldots$. . . . . . . . . 60

5.1 Tracking error $y(t)-y_{d}(t)$ for $y_{d}(t)=\frac{2 \pi}{180} \sin t$ : SISO spacecraft model with fuel slosh. . . . . . . . . . . . . . . . . . . . 71

5.2 Tracking error $y(t)-y_{d}(t)$ for $y_{d}(t)=\frac{2 \pi}{180} \sin t+\frac{4 \pi}{180} \cos t$ : SISO spacecraft model with fuel slosh. . . . . . . . . . . . . . . . . . . . 72

5.3 Input $u(t)$ for the tracking error $y(t)-y_{d}(t)$ shown in Figure 5.2. . . 72

5.4 Output response for $y(t)=\theta(t)$ and $y_{d}(t)=\frac{2 \pi}{180} \sin t$ : SISO spacecraft model with fuel slosh. . . . . . . . . . . . . . . . . . 73

5.5 Slosh angle response $\psi(t)$, for $y_{d}(t)=\frac{2 \pi}{180} \sin t$ : SISO spacecraft model with fuel slosh. . . . . . . . . . . . . . . . . . . 73

5.6 Modified MIMO Spacecraft with fuel slosh dynamics [53] . . . . . . . 74

5.7 Tracking error $y(t)-y_{d}(t)$ for the reduced order MIMO spacecraft model with fuel slosh. . . . . . . . . . . . . . . . . . . 87

5.8 Slosh Angle $\psi$ Time Response. . . . . . . . . . . . . . . . . . . . . . 88

5.9 Attitude Angle 6: Output Time Response. . . . . . . . . . . . . . 88 
5.10 Transverse Velocity $v_{z}$ : Output Time Response. . . . . . . . . 88

5.11 Transverse Control Force $F_{T}$, Pitching Moment $M$, and Gimbal Deflection Angle $\delta \ldots \ldots \ldots \ldots \ldots$

6.1 Approximation based smooth adaptive inverse compensation control. 93

6.2 System state tracking errors with input nonlinearities. . . . . . . . 106

6.3 System state tracking errors without input nonlinearities. . . . . . . 106

6.4 System state tracking errors with partial input nonlinearities. . . . . . 107

6.5 System state tracking errors with partial input nonlinearities and adaptive compensation. . . . . . . . . . . . . . . . 107

6.6 Control signal $\mathrm{u}(\mathrm{t}) \ldots \ldots \ldots \ldots$

6.7 System output (solid) and reference signal (dotted): angle of attack $(\mathrm{rad}) \ldots \ldots \ldots \ldots \ldots \ldots \ldots \ldots$

6.8 Tracking error during the first $8 \mathrm{~s}$ (the dashed lines indicate the required error bound). . . . . . . . . . . . . . . . . . . . 109

6.9 System output with robustifying signal (solid), without (dotted): angle of attack $(\mathrm{rad}) . \ldots \ldots \ldots \ldots \ldots$

6.10 System response of regulation: $\mathrm{r}(\mathrm{t})=0 . \ldots \ldots \ldots \ldots$ 


\section{Chapter 1}

\section{Introduction and Overview}

Uncertain dynamics in modeling nonlinear systems is one of the primary challenges in the research and development of next-generation adaptively controlled systems. These parametric uncertainties and unknown modeling effects inherent in real-life dynamical systems can cause undesirable system failures and deteriorated control system performance. To help improve the level of achievable control system performance in highly uncertain nonlinear dynamical systems, approximation based adaptive control methodologies can be implemented as a strong tool to estimate the system parametric uncertainty and approximate the unknown system nonlinearities in a refined control framework structure. Approximation based adaptive control is in essence a combination of a number of different concepts from dynamical systems theory, stability theory, function approximation techniques, parameter estimation methods, nonlinear control designs, and an intuitive design mechanism to apply all of these tools in a unified methodology [19].

\subsection{Approximation Based Control Methodology}

The motivation behind approximation based control is the ability to improve online control performance by accurately determining uncertain nonlinear portions of the 
system model via function approximation methods. Although, the available model information maybe limited a priori, additional information can be updated while the system is operating. The concept of function approximation has been presented in a general context for both theory and practical purposes [54]. Originally formulated in [42], the idea of employing neural network structures for approximation and control instigated further research in the area of approximation based control methods. Although the majority of the initial results in this field were based on the neural network architecture [47] for function approximation models of the unknown nonlinearities, neural networks only represent just one class of many different types of function approximators. For example, radial basis functions, polynomials, rational functions, fuzzy logic systems, wavelets, gain scheduling and spline functions, are all function approximators that have been applied in a similar context $[8,46,18,57]$. Therefore, control methodologies based on these approximation models with adaptivity online features are referred to as approximation based adaptive control. In contrast to adaptive nonlinear control where the uncertainty is in the unknown parameters, approximation based adaptive control deals with system uncertainty that is due to unknown nonlinearities [49].

There are two main types of parameter estimation methods: the so called online learning technique and the offline tuning approach [19]. Online parameter estimation methods are based on the idea of choosing suitable initial estimates for the unknown parameters, then updating the parameter estimates using the current set of measurements available. The three steps involved in the online learning design procedure are fairly simple, first derive a parametric model that rewrites the system into a model of known structure and unknown parameters. The second step is to design an online learning scheme, this consists of replacing the unknown parameters in the parametric model by some adjustable set of parameters. The third and final step is to derive an adaptive law for updating the adjustable parameters in the newly formed adaptive approximator. The adaptive law is typically based on a chosen parametric error 
signal that is used in an optimization method minimizing a chosen type of cost function. The offline parameter estimation tuning approach first requires the collection of all necessary input-output data to be processed and then fit to a predetermined or assumed model. There are a number of different approximation based methods that can be used to determine the best fit for the model data each of which has it's own benefits and drawbacks [54].

To illustrate the motivation for function approximation and control we present a simple example of a single-input single-output (SISO) continuous time system. The control objective in this case is for the system output signal $y(t)$ to track a desired signal $y_{d}(t)$ such that the tracking error $e(t)=y_{d}(t)-y(t)$ is forced to zero. Consider the 1-dimensional state system representation as

$$
\begin{aligned}
\dot{x}(t) & =f(x(t))+u(t), \\
y(t) & =x(t)
\end{aligned}
$$

where $x(t) \in \Re^{1}$ is the single system state, $u(t) \in \Re^{1}$ is the single control variable input, $y(t)$ is the measurable output signal, the time $t \geq 0$ and $f(x)$ is the unknown function representing the dynamics. A simple choice of $u(t)$, applying a cancellation of terms methods for asymptotic tracking, yields the control law

$$
u(t)=-\lambda e(t)-\hat{f}(x(t))+\dot{y}_{d}(t)
$$

where $e(t)=y_{d}(t)-y(t)$ is defined as the system output tracking error. The design constant $\lambda$ is used to ensure stability, $\hat{f}(x)$ is the approximation of the unknown function $f(x)$, and we follow the assumption that the derivative $\dot{y}_{d}(t)$ is continuous, bounded, and available without the specific need for explicit differentiation of the tracking signal $y_{d}(t)$.

Next, to determine the system stability, we solve for the closed-loop tracking error dynamics from

$$
\begin{aligned}
\dot{e}(t) & =\dot{y}_{d}(t)-\dot{y}(t), \\
& =\dot{y}_{d}(t)-f(x)-u(t) .
\end{aligned}
$$


Substituting the control law from (1.2) into the system equation (1.1) and by cancellation of like terms yields the following form for the closed-loop tracking error dynamics:

$$
\dot{e}(t)=\lambda e(t)-(f(x)-\hat{f}(x)) .
$$

The first case of interest is when $\hat{f}(x) \neq f(x)$ and this shows how the tracking error performance is directly affected by the accuracy of the approximated model function $\hat{f}(x)$. For the other case when $\hat{f}(x)=f(x)$, the error dynamics simplify to

$$
\dot{e}(t)=\lambda e(t)
$$

and will be stable for values of $\lambda<0$. For the purposes of numerical simulation in MATLAB we start by assuming we have some priori information about the system function, so that we choose the unknown function to be $f(x(t))=\cos x(t)$, with no approximation $\hat{f}(x)=0$ function, and the desired tracking signal as $y_{d}(t)=\pi \cos 0.2 t$ for a time scale of $t \in[0,100]$. Figure 1.1 shows the system performance and tracking error for the case where there is no function approximator $\hat{f}(x)=0$.
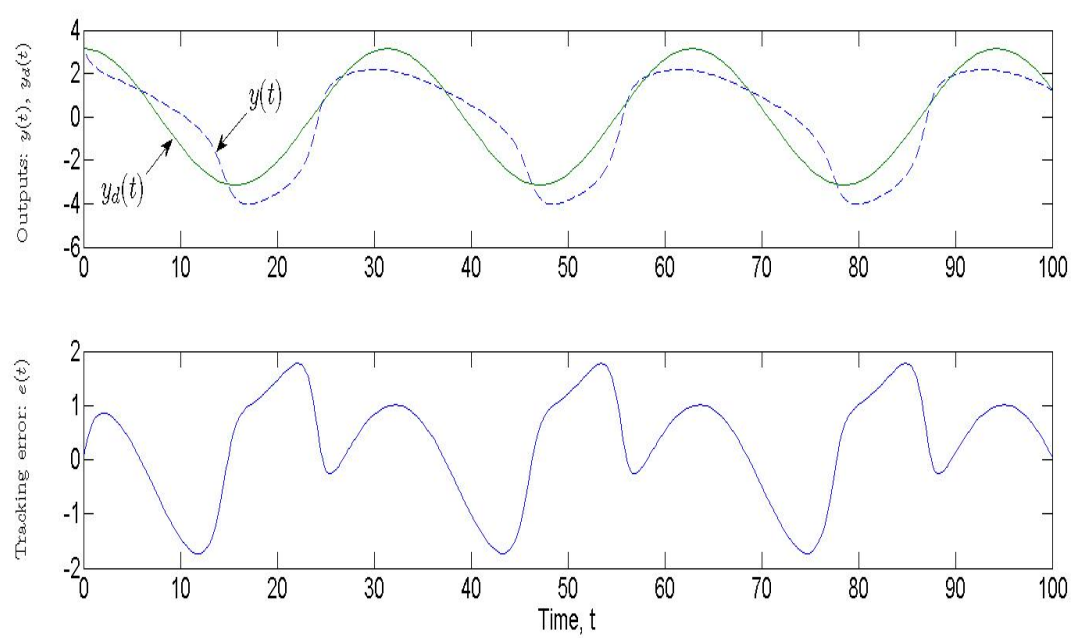

Figure 1.1: Closed-loop system output tracking performance for the control law (1.2) applied to the system equation in (1.1) with no function approximator $\hat{f}(x)=0$. 
The top plot of Figure 1.1 reveals how the system tracking performance is affected by the accuracy of the design model $\hat{f}(x)$ used in the simulation. For the next simulation we assume the same priori information for $f(x)$, but instead we choose an approximating function for $\hat{f}(x)$ that is close to this function (e.g. $\hat{f}(x)=0.7 \cos x$ ), based on the priori information. Figure 1.2 shows the results for the simple approximationbased control system with priori information used for function approximation purposes.
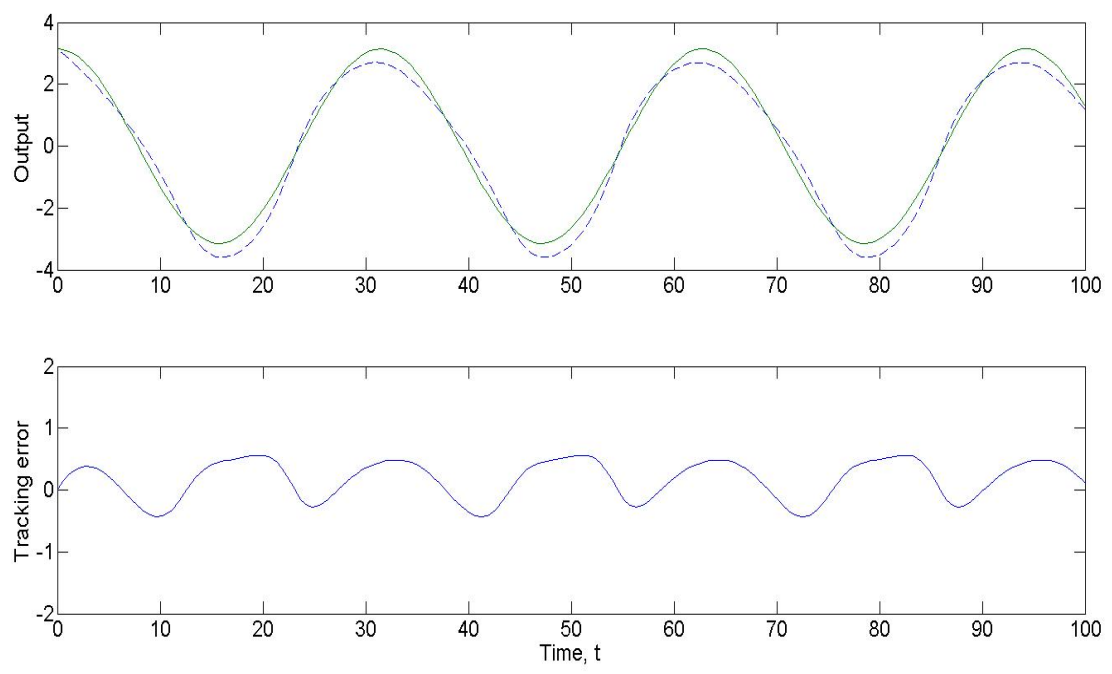

Figure 1.2: Output tracking performance for (1.2) applied to (1.1) with function approximation $\hat{f}(x)=0.7 \cos x$, the solid line is the desired reference trajectory $y_{d}(t)$ and the dashed line is the system output response $y(t)$. The tracking error is plotted on the bottom part of the figure.

This simple example motivates us to pursue more advanced methods of model approximation for control purposes. Although, we assumed there was some priori information used in the structure, this is not usually true. The effect of modeling accuracy is pronounced in the tracking error and must be reduced to improve the overall level of achievable control system performance. As we will see later in this dissertation that such approximation models exist and can be used for these purposes. 
In the next section, we present some relevant background information on the topic of spline function theory and how it can be used as a function approximator for the purposes of achieving specific control objectives.

\subsection{Literature Overview}

As the next generation of nonlinear control system devices are employed to meet new and more complex objectives, the uncertainties associated with these type of systems are becoming an increasingly important challenge to the development of control schemes that can achieve desired system performance.

\subsubsection{Aircraft Control with Synthetic Jet Actuators}

Historically, the development of aircraft control devices have been bulky mechanical structures that provide desired performance throughout the aircraft flight envelope. As the next generation of sophisticated aircraft control system devices are employed to meet new and more demanding flight objectives, the uncertainties associated with these devices become an increasingly important challenge to the development of control schemes that can achieve desired system performance. A specific area of interest recently is in the use of active flow control devices, such as synthetic jet actuators to improve the aircraft aerodynamic performance. To implement synthetic jet actuators, which have inherent nonlinearities, requires an understanding of the actuator nonlinearity and development of an effective control methodology to handle them. One such technique is approximation-based adaptive inverse compensation to cancel the unknown smooth actuator nonlinearities, such that full control authority is accomplished and maintained throughout the complete operation time.

In the literature [6] and [71], synthetic jets have been proven to be an efficient method in controlling separated flow, resulting in an increase in lift and a delay in stall. Some of the main advantages of synthetic jet actuators are their low cost, 
compact structure, ease of operation and the fact that they are zero net mass flow in nature meaning that they produce a jet without the need for an external fluid injection. This concept of a zero net mass flow jet is created by the transfer of linear momentum from the internal oscillating actuator in the cavity through the orifice to allow for flow control without net mass injection. Typically, synthetic jet actuators are made up of a piezoelectrically driven membrane that produces a synthetic jet flow. Interestingly enough, recent studies [58] have demonstrated flow control with plasma synthetic jet actuators.

As shown in [13], the control effect of a synthetic jet actuator is a nonlinear function of both the applied input to drive the piezoelectric diaphragm to generate the air flow and the aircraft's angle of attack $\alpha$. This feature is important to consider when designing an adaptive compensation scheme to cancel the effect of actuator nonlinearities inherent in the control of an aircraft system with synthetic jet actuators. In this dissertation document, we extend these results to apply such modeling and adaptive control techniques to multiple input commercial transport aircraft model with smooth nonlinear synthetic jet actuator characteristics. The system dynamics include the aircraft's angle of attack for the study of adaptive approximation-based compensation of the unknown smooth actuator nonlinearities. An important issue of an approximation-based adaptive compensation control scheme [63] is determining a suitable parameterization for the synthetic jet actuator nonlinearity and its inverse. Given that the actuator nonlinearity and its inverse are highly nonlinear, they are best to be approximated.

In this dissertation, we address this issue by approximating the smooth synthetic jet actuator nonlinearity and its inverse with multivariable spline functions. In our previous results [66] we dealt with the case where the nonlinearities were non-smooth and may have contained discontinuities. Here we assume that the nonlinearities are smooth functions. Spline functions are a good fit for adaptive control applications because they are linear in their parameters, which are the spline coefficients. In 
addition, splines have been proven useful for signal and image processing [77]. More recently, spline functions have been applied to approximate feedback linearization. The motivation for this approach has been for systems that fail to meet the necessary conditions required for feedback linearization, also to reduce the algebraic complexity encountered with pseudolinearization [8]. The contributions of this development are two-fold: spline function network development for adaptive inverse compensation of smooth synthetic jet actuator nonlinearities applied to a multiple input commercial aircraft model, and performance analysis for such an adaptive approximation-based control design scheme.

\subsubsection{Modeling and Control of Spacecraft with Fuel Slosh}

Slosh-tolerant spacecraft flight control designs have been the focus of numerous research developments dating as far back as 1966. When Abramson published his monograph [1] that had summarized all that was then known about sloshing liquids and how they affect spacecraft dynamics, the print run of several thousand copies was quickly distributed and proved to be extremely popular [16]. From the serious loss of the ATS-V spacecraft in 1969 to the unsuccessful firing of the NEAR spacecraft in 2000 [78], and more recently in 2007 a spacecraft company Space X launch vehicle failed due to sloshing propellant in one of the tanks [87]. Most of the problems reported on-board control systems that had sensor readings with higher than expected lateral accelerations from the fuel slosh. Even more recently, with the retiring of the well-known NASA space shuttle, researchers are developing alternative spacecraft models and opening new opportunities for advanced space flight control technology.

Mathematically approximated models are important to the spacecraft industry not only for control purposes, but also due to the immense amount of monetary spending that is needed to build and test a real spacecraft. The models are used mainly for computer simulation to evaluate specific performance objectives prior to physical manufacturing. Models can also be verified experimentally, reconstructed 
from certain conditions at the time of failure, and studied by applying different design strategies. Spacecraft control and model simulation is a very complex topic, but with accurate approximations the task can be accomplished successfully.

To model a system undergoing liquid slosh disturbance, one class of design methods first developed by Graham, consists of equivalent mechanical system models to approximate the liquid sloshing and this makes it possible to be included into the spacecraft dynamics [25]. Furthermore, the accuracy of the approximated models were verified via experimental analysis: experiments were performed to verify the models $[1,75,78]$. Subsequently experiments to model and control the free surface liquid phenomenon led to the advent of computer simulations and damping fixtures that are still in use today [16]. The quest to include the liquid sloshing into the spacecraft dynamics had been met; however a practical method to control and verify the system had not.

There have been a number of adaptive control designs for spacecraft $[5,7,17,29$, 43, 44]. The motivation of these designs has been to identify the inertia matrix for the spacecraft dynamics. With current modeling designs a spacecraft inertial matrix is only known within a 5\% modeling error [79]. In addition, designs like the International Space Station which require in orbit assembly; throughout the assembly process the system inertia matrix will change and the system controller must be designed in such a way to account for this change.

Adler et al [2], developed an adaptive control system for propellant slosh for launch vehicles. The adaptive system utilized a laser sensor to measure fluid displacement in the tank, a recursive-least squares estimator to estimate the natural frequency of the sloshing fuel, and a linear filter which stabilizes the unstable fuel slosh using the frequency estimate. The method was successful for a single-pendulum model and requires no tank baffles or partitions. This result gives encouragement for further development into more complex adaptive control systems such as, spacecraft with fuel slosh dynamics and multiple mass-spring approximations to improve the accuracy of 
the system equations even further.

An expanded study of both approximation-based adaptive control and spacecraft with fuel slosh dynamics has not yet appeared in the literature; we have thus chosen to develop the scenario as part of this Dissertation. The main achievements of our research are to develop novel approximation based adaptive control designs and analyze their ability to effectively handle any unknown nonlinearities and parameter uncertainties inherent in nonlinear control systems with applications to real-time space and aircraft flight. The key features of the developed adaptive control schemes will be the use of spline functions for approximation purposes, the guarantee of desired system stability and tracking properties under deteriorated control authority caused by unknown system nonlinearities and parameter uncertainties. These results yield a framework of design guidelines for control performance objectives under unknown system nonlinearities and parameter uncertainties with respect to nonlinear systems (e.g. spacecraft and aircraft control applications).

\subsection{Contributions Summary}

As presented in the previous sections, the contributions discussed throughout this dissertation aim to expand the study of approximation based adaptive control theory using spline functions with applications to nonlinear dynamical systems.

Employing spline functions for nonlinear dynamical system applications, advanced system modeling and control techniques are developed forth and analyzed. First, various spacecraft with fuel slosh models are presented and studied for both approximation method development and adaptive control structure design for future nonlinear systems. In particular, we show analytical proofs and mathematical simulations that spline function approximation based adaptive control can reduce the tracking error for complex objectives, a common measure of system performance (Chapter 5). Application to aircraft with synthetic jet actuator nonlinearities is also developed, 
analytically evaluated control error, and supplemented with mathematical system modeling verification simulations using real life physical parameters (Chapter 6).

In Chapter 3, new developments on spacecraft with fuel slosh modeling techniques are proposed through a modified multi-mass pendulum sloshing modes approximation based model with multiple inputs. This advanced modeling technique would improve the accuracy of the system model in a way that would reduce the errors during experimental testing caused by various mathematical approximations and tracking performance evaluations. First, a simple single input spacecraft with fuel slosh model is presented to satisfy the state variable form conditions to allow for control input development and spline function based approximation design. Next, a pendulum assumption based multiple input spacecraft model is formulated into state variable form and analyzed for stability attributes. The context of these models are shown to be valuable during control system design by applying a more accurate engineering solution to the modeling design challenge.

Finally, contributions to advanced approximation methods in nonlinear dynamic models are made through developments of typical nonlinear system models employing spline functions in an adaptive control context (Chapter 5), in addition to adaptive feedback control for aircraft applications (Chapter 6). Approximation based adaptive control using spline functions for a spacecraft with fuel slosh nonlinear dynamic model implemented under the pendulum assumption is first studied in Chapter 3 and developed further with design parameters in Chapter 5. It is determined that a spline function based approximation methods under adaptive control techniques for nonlinear systems can reduce the tracking error to a small residual set for a reasonable number of mathematical computer calculations. The contributions in Chapter 4 are an improved actuator nonlinearity characteristic modeling approximation study of aircraft with synthetic jet actuator inputs.

Overall, the contributions presented in this dissertation document include adaptive control techniques implemented through spline function based approximation 
methods for next-generation spacecraft systems and aircraft flight control, with the objective of maintaining stability demands in future spacecraft systems and achieving desired tracking performance under actuator nonlinearities in new-age aircraft design methods for control system implementations.

\subsection{Dissertation Preview}

The remaining dissertation document is organized as follows. In Chapter 2, we describe the general problem formulation and go through the relevant technical background of our developed research topics. In Chapter 3, we present the various spacecraft with fuel slosh models developed from the literature that were chosen to be applied in our study. Synthetic jet actuator technology applied to aircraft control design and modeling techniques are summarized in Chapter 4. We then present our results for approximation based adaptive control using spline functions applied to various spacecraft with fuel slosh control models in Chapter 5. Adaptive compensation of synthetic jet actuator nonlinearities for aircraft control is developed in Chapter 6, including modeling and simulation results for a real-life aircraft dynamic model. Finally, conclusions and a brief technology outlook for future implementations are suggested in Chapter 7. 


\section{Chapter 2}

\section{Technical Background and Problem Formulation}

System dynamic uncertainties can cause undesirable parametric, structural and environmental nonlinearities, which require to be accounted for in order to achieve the desired system performance and maintain the control objective. Design issues involved in approximation based adaptive control systems are controller parametrization methods, deriving a set of error dynamics, choosing adaptive update laws, analyzing stability and tracking properties. These issues become more challenging, because of inherent system structural uncertainties and unmodeled parametric nonlinearities.

\subsection{Splines for Approximation and Control}

In the mathematical field of function approximation, a spline function is a special type of interpolation that involves connecting piecewise polynomials through a set of data points. The result is essentially a curve that is pieced together by polynomial segments that are subject to continuity constraints at the connecting points. For example, if we simply connected the data points with straight lines $\left(1^{\text {st }}\right.$ order

polynomials), this would be considered a continuous spline of order two interpolating 
over the data set. Connecting the data set with $2^{\text {nd }}$ order polynomials in such a way that the graph has a continuous first derivative at these interconnecting points is a spline of order three [19]. A spline function is defined on a chosen interval and can be used to approximate complex mathematical functions defined by a specified set of data points. Therefore, splines are composed of pieces of simple functions defined on subintervals and joined at the endpoints [90]. The term "splines" was first introduced in the mathematical literature in 1946 [61]. Since this time there have been a number of monographs that have summarized the theory of splines and how they can be used for approximation and control $[4,62,55]$. More recently, spline functions have been applied to approximate feedback linearization. The motivation for this approach has been for systems that fail to meet the necessary conditions required for feedback linearization. In addition, spline functions are used to reduce the algebraic complexity encountered with pseudolinearization [8]. They are superior to polynomial interpolation, because of the interesting fact that the approximation error can be made smaller without having to resort to applying higher order polynomials. The main challenges with spline functions are minimizing approximation error, deriving an optimal set of approximating parameters and choosing a suitable number of intervals to spline over.

Basis splines (B-splines) are important in that they can represent any spline function of the same degree by a linear combination from the set of basis elements; in particular, the Cardinal B-Spline for intervals of the form $[-1,0,1,2, \ldots]$. Splines have been used to approximate system functions for nonlinear systems that are very complex and otherwise would require substantial algebraic computation. By representing the complex system functions with splines we can reduce the mathematical computation required to execute the objectives and improve the accuracy of the system modeling representation. The most widely used in today's applications are the cubic splines, due to their minimum curvature property [77]. The concept of an ideal measure of curvature is considering the spline functions continuous second-order derivative and taking it's squared norm along the path of the function, this is a rough 
approximation to the potential energy of the original draughtsman's spline used in engineering drawings and maps.

In this Dissertation, the splines we chose to consider are defined as symmetrical, bell shaped functions constructed from the functions $g_{k}: \Re^{1} \rightarrow \Re^{1}$, defined recursively [19], by

$$
\begin{aligned}
g_{k}(x) & =\int_{0}^{1} g_{k-1}(x-\lambda) d \lambda, \\
& =\int_{-\infty}^{\infty} g_{k-1}(x-\lambda) g_{1}(\lambda) d \lambda,
\end{aligned}
$$

for $k>1$. This defines the Cardinal B-spline of order $k$ (degree $k-1$ ) for the knot at 0 , where

$$
g_{1}(x)= \begin{cases}1 & 0 \leq x<1 \\ 0 & \text { otherwise }\end{cases}
$$

For example, a cubic Cardinal B-spline of order 4 and degree 3 , for the knot at 0 is given by [80]

$$
g_{4}(x)=\left\{\begin{array}{lc}
\frac{x^{3}}{6} & 0 \leq x<1, \\
\frac{-x^{3}}{2}+2 x^{2}-2 x+\frac{4}{6} & 1 \leq x<2, \\
\frac{x^{3}}{2}-4 x^{2}+10 x-\frac{44}{6} & 2 \leq x<3, \\
\frac{-x^{3}}{6}+2 x^{2}-8 x+\frac{64}{6} & 3 \leq x \leq 4, \\
0 & \text { otherwise. }
\end{array}\right.
$$

The B-spline basis elements of order $k$ for the knot at $x=j$ is $g_{k j}(x)=g_{k}(x-j)$, which is simply just a translation of the original B-spline $g_{k}(x)$. The basis elements have support for $x \in(j, k+j)$, and to form a partition of unity on $x \in(0,1)$ the sum of the B-spline basis elements are $\sum_{j=0}^{n} g_{k}(x-j)=1$, this reveals how each corresponding parameter only influences the curve for that knot point and interval of support. For the cubic spline case $(k=4)$, we have plotted these basis elements shown in Figure 2.1.

The cubic function $s_{k}(x)=\sum_{j=1-k}^{n-1} \theta_{j} g_{k}(x-j)$ is a spline of order $k$, that has $(n+k-1)$ knots at the points $x=1-k, 1,2, \ldots, n-1$, with the coefficients $\theta_{j}$. The 


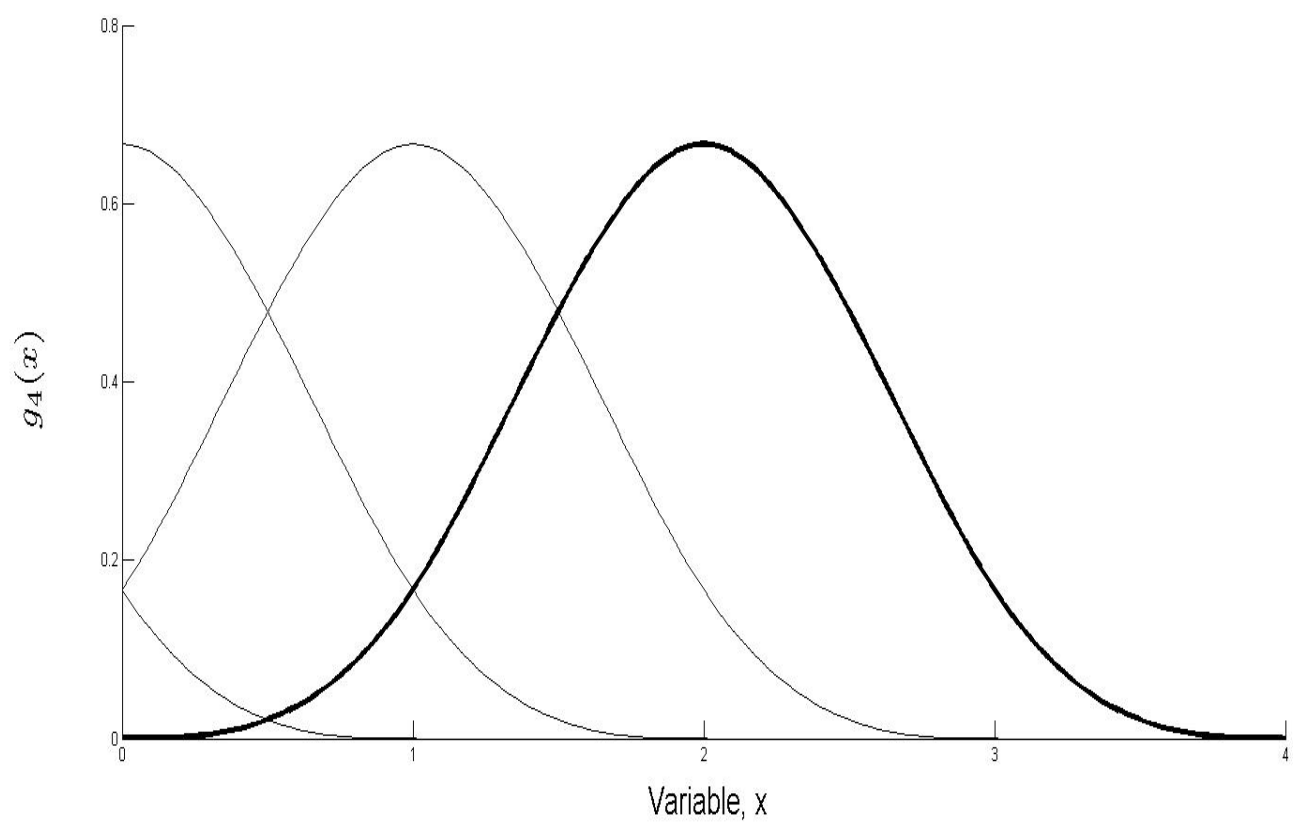

Figure 2.1: Cubic B-spline basis elements for (2.2).

set of basis elements $\left\{g_{k}(x-j)\right\}_{j=1-k}^{n-1}$ form a partition of unity on $[0, n]$. Instead, if we write the basis elements as

$$
\phi_{j}(x)=g_{k}\left(n \frac{x-a}{b-a}-j\right)
$$

for $j=1-k, \ldots, n-1$, then the new basis set is $\left\{\phi_{j}\right\}_{j=1-k}^{n-1}$, that is formed by translating and dilating the $\mathrm{k}$-th order Cardinal B-spline, we form a partition of unity on $[a, b][19]$. Defining the approximator as

$$
\hat{f}(x ; \theta)=\theta^{T} \phi(x)=\sum_{j=1-k}^{n-1} \theta_{j} \phi_{j}(x),
$$

with $\phi_{j}(x)$ as defined in (2.3), we have direct access to adjust the parameter vector $\theta$ of the approximator without having to satisfy other continuity or matching constraints.

By approximating the uncertain nonlinearity modeling effect with spline functions, we enable the design of an approximation based adaptive control technique and improve the accuracy in approximating the uncertain model parameters required 
to meet the control objectives. To demonstrate the concept of spline function approximation we present a simple example to approximate the function $f(x)=\cos (2 x)$ for the time interval $x \in[0,5]$. The data points are generated using MATLAB and then fed through the spline network in (2.2) to formulate the approximator function $\hat{f}(x ; \theta)$. From $(2.3)$ we choose $k=4$, over the interval $[a=0, b=5]$, with $n=5$, this yields the index variable $j=-3,-2,-1,0,1,2,3,4$, which for the sake of notation can be interchangeable with the corresponding index $j=1,2,3,4,5,6,7,8$ when we are counting the number of basis elements. Therefore, this function approximation structure has 8 coefficients with 8 basis elements. Figure 2.2, shows the function $f(x)$ with it's approximator function $\hat{f}(x ; \theta))$ plotted as a dashed line, the bottom part of the figure shows the spline basis functions $\phi_{j}(x)$ used in the approximation structure.

The most apparent challenge in this simple example is choosing the optimal parameter vector $\theta=\left[\theta_{1}, \theta_{2}, \theta_{3}, \theta_{4}, \theta_{5}, \theta_{6}, \theta_{7}, \theta_{8}\right]^{T}$ that generates the closes fit of $\hat{f}$ to $f$. Following (2.4), with the chosen numerical values results in the spline function approximation network

$$
\hat{f}(x ; \theta)=\sum_{j=1}^{8} \theta_{j} \phi_{j}(x)=\theta^{T} \phi(x)=\phi(x)^{T} \theta,
$$

where $\phi(x)=\left[\phi_{1}(x), \ldots, \phi_{8}(x)\right]$. The problem of solving for $\theta$ such that $\hat{f}(x ; \theta) \approx f(x)$ we choose $m$-distinct points that satisfy the matching condition

$$
\left[\begin{array}{c}
\phi\left(x_{1}\right)^{T} \\
\phi\left(x_{2}\right)^{T} \\
\vdots \\
\phi\left(x_{m}\right)^{T}
\end{array}\right]\left[\begin{array}{c}
\theta_{1} \\
\theta_{2} \\
\vdots \\
\theta_{m}
\end{array}\right]=\left[\begin{array}{c}
f\left(x_{1}\right) \\
f\left(x_{2}\right) \\
\vdots \\
f\left(x_{m}\right)
\end{array}\right]
$$

which can be seen as $\Phi^{T} \theta=F$, where $\Phi=\left[\phi\left(x_{1}, \ldots, \phi\left(x_{m}\right)\right]\right.$. If we choose $m=8$, such that we have the same number of basis functions and the matrix $\Phi^{T}$ is nonsingular, then the unique solution for $\theta$ is

$$
\theta=\left(\Phi^{T}\right)^{-1} F
$$


Although this matching condition is limited to the case where the number of data points is equal to the number of coefficients and $\Phi^{T}$ being nonsingular, it gives some insight into the complexity of solving for an optimal $\theta$. To display the effectiveness of the spline function approximation method we also apply it to the simple example presented in Section 1.1. Consider the approximating structure from the previous example with a different set of parameters $\theta$ and basis functions $\phi_{j}(x)$, where $j=1,2,3,4,5,6,7,8$ as in the previous example. MATLAB simulation results are displayed in Figure 2.4, where there is a drastic reduction of the system output tracking error with the spline function approximator $\hat{f}(x ; \theta)=\theta^{T} \phi(x)$. Although we have only considered periodic functions in these introductory examples, the concept of using splines for functional approximation can extend to more complex non-periodic functions that we will present later in this document.
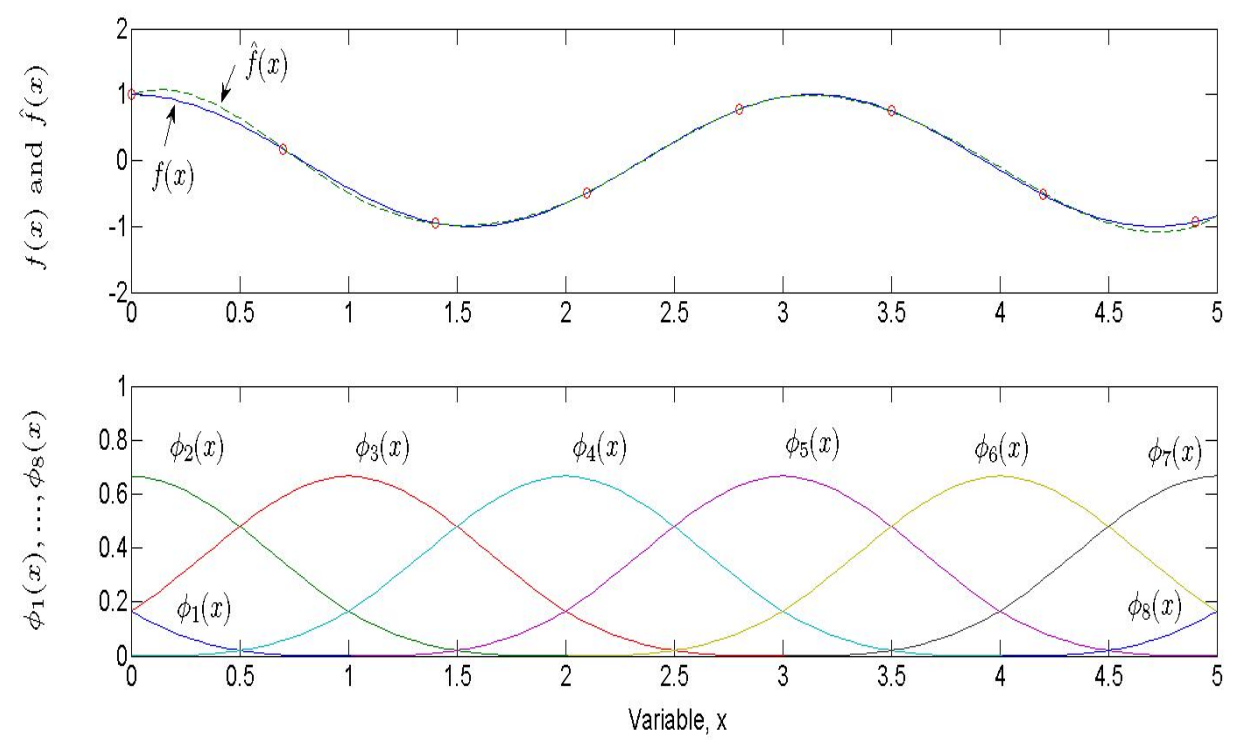

Figure 2.2: The solid line in the top plot shows the desired function $f(x)=\cos 2 x$ and the dashed line is the spline function approximator $\hat{f}(x, \theta)$. The basis functions $\phi_{1}, \ldots, \phi_{8}$ are plotted on the bottom part of the figure.

Although these simple examples only consider a univariate spline basis, later we 
will present a multivariable spline function basis that will consist of the two dimensional basis elements shown in Figure 2.3.

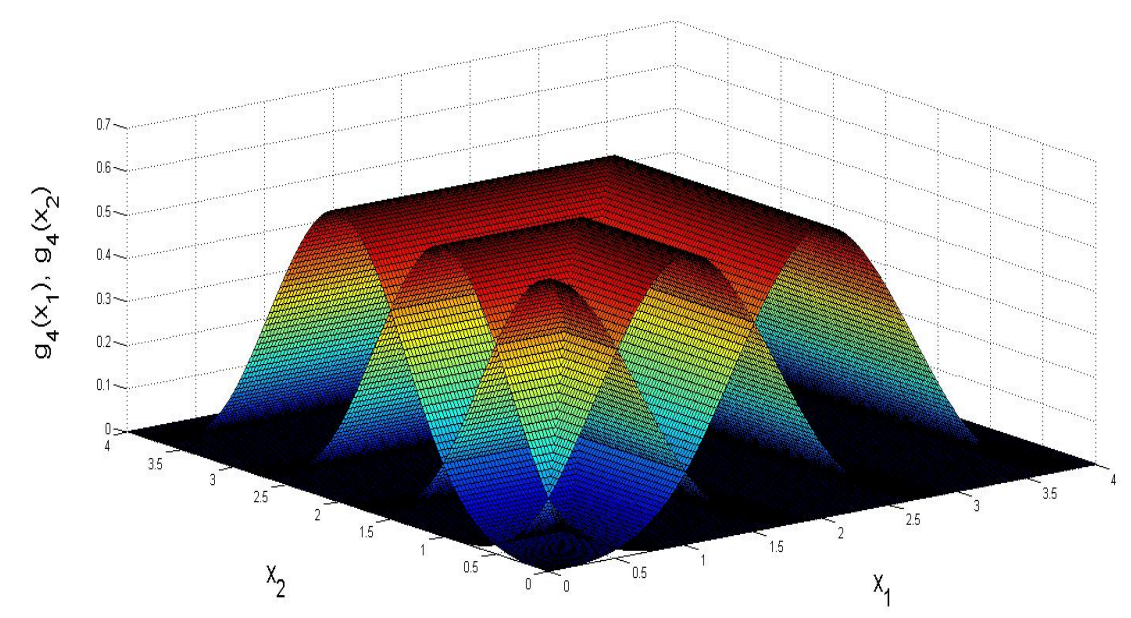

Figure 2.3: Multivariate cubic B-spline basis elements.
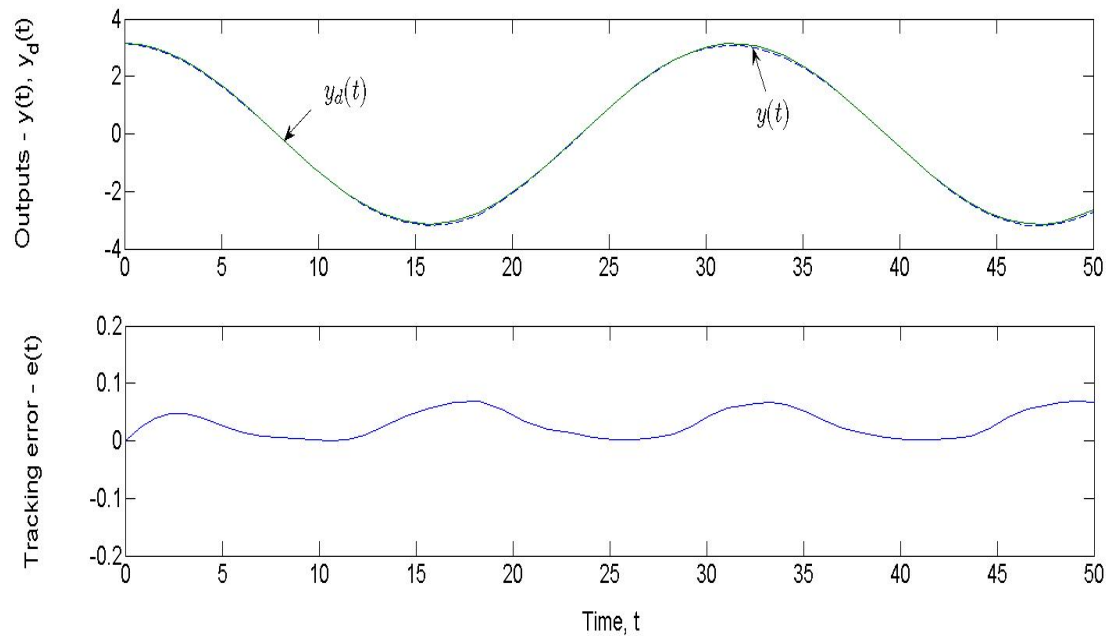

Figure 2.4: The solid line in the top plot shows the desired output $y_{d}(t)=\cos t$ and the dashed line is the actual output response $y(t)$ with the spline function approximator $\hat{f}(x ; \theta)$. The tracking error $e(t)$ is plotted on the bottom part of the figure.

As we will see in the next section we can employ an adaptive control method to 
update the parameter vector $\theta$ in the spline function approximator structure.

\subsection{Approximation Based Adaptive Control Schemes}

Adaptive control theory provides a strong tool to assist in the design of feedback control systems. Typically these systems contain parametric, structural, and environmental uncertainties that might be completely unknown. The basic principles of the methodology is to start by estimating the unknown system parameters online using a proper adaptive update law estimation method (e.g. gradient optimization), and then use the estimates in place of the unknown parameters in the feedback control law. As the fuel level of the spacecraft drops and the tank becomes partially filled the remaining liquid sloshing around in the tank will cause parametric uncertainties. The varying degrees of nonlinearity due to this highly complex liquid motion results in structural uncertainties, and external space objects are some hazardly environmental uncertainties, these are just some of the many challenges in spacecraft control system design. Similarly, for aircraft with synthetic jets, they suffer from unknown actuator nonlinearities, unmodeled dynamics and environmental uncertainties.

Adaptive control is a widely practiced methodology from the family of feedback control systems. Motivated by the new feedback technologies that were invented during World War II, including automatic pilot, gun positioning, and radar control systems. In 1957, Drenick and Shahbender introduced the term adaptive control. As the 60 's came to an end, adaptive control had begun seeing many advancements in stability analysis for linear systems [41]. Although, these initial results assumed that all system uncertainty was due to unknown parameters, it opened the door for control designers to make modifications to the existing adaptive algorithms to account for other types of uncertainties (i.e. external disturbances, signal measurement noise). In the last two decades, most of the efforts in adaptive control have been towards nonlinear systems and have experienced many great advancements [32]. Modern 
applications for adaptive control found in the literature include temperature control, chemical reactor control, automobile control, artificial heart control, robot control, aircraft control, and physiological control.

A typical adaptive control system (see Figure 2.5) consists of unknown plant parameters, where the plant is the system process that the adaptive controller is designed to control. Adaptive update laws to generate the unknown parameter estimates. The designed feedback controller and a model reference system for tracking objectives.

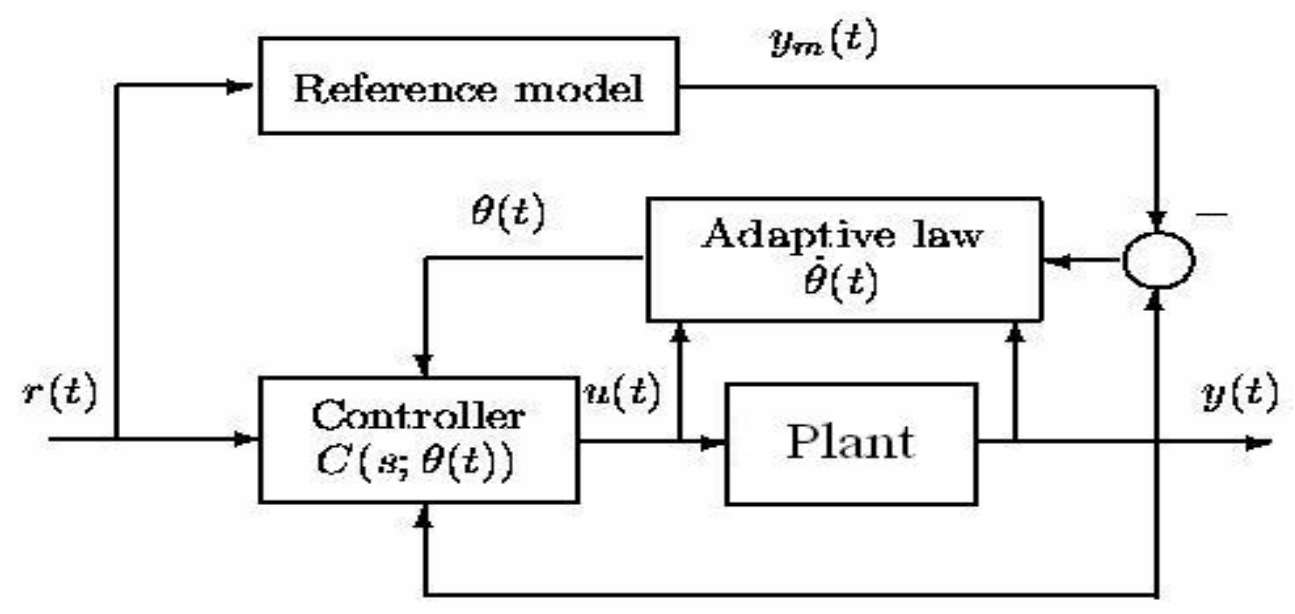

Figure 2.5: Basic model reference direct adaptive feedback control.

There are two popular methods of combining the parameter estimates with the feedback control law to create the adaptive controller. The first strategy, known as direct adaptive control, uses the parameter estimates directly to determine the controller parameters. The second method, referred to as indirect adaptive control, the parameter estimation algorithm first estimates the unknown plant parameters and then maps the estimates to the controller parameters through a strategically determined design equation. A direct adaptive control design utilizes a direct estimation of the controller parameters from an adaptive law. The two methods for direct designs are Lyapunov and Gradient. In contrast, an indirect approach calculates the controller parameters from an algebraic design equation using the initial estimates of the plant parameters. With direct adaptive control the controller parameters are 
updated directly. As a subset of these designs we have model reference and pole placement. Model reference designs are used when the zeros of the system are stable $R e[s]<0$. When the system zeros are unstable $R e[s] \geq 0$, they are either canceled out by corresponding poles or pole placement designs are used to place the system poles at a desired location. The complexity of the design varies by the order of the system, as well as the number of system inputs and outputs.

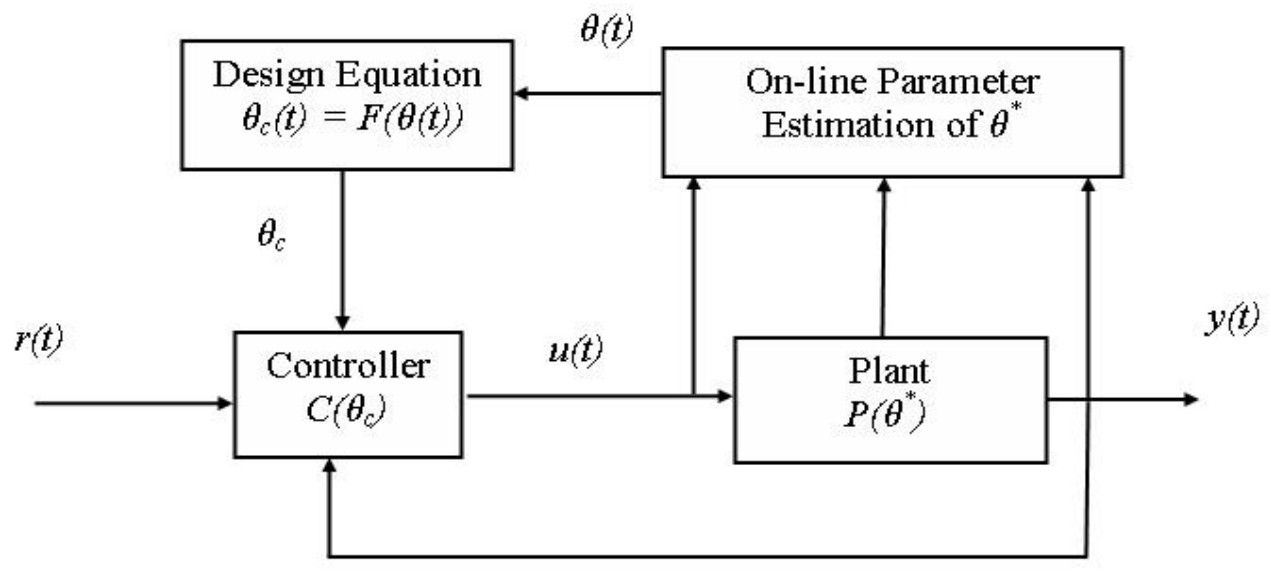

Figure 2.6: Indirect adaptive control system.

When the system is a multi-input multi-output (MIMO) system the transmission zeros are considered to determine which adaptive control design procedure to follow, the same stability conditions are considered as described for the single-input single-output (SISO) systems. Some of these approaches will be presented briefly to introduce the basic equations including the controller structure and design assumptions. As an expansion of the adaptive control methodology, approximation based adaptive control deals with cases where the system uncertainty also includes unknown nonlinearities that need to be approximated, in addition to the estimated unknown system parameters. Generally, a nonlinear adaptive control design deals with systems where the uncertainty is due to unknown system parameters that appear linearly with respect to known nonlinearities. Therefore, adaptive approximation based control can be viewed as an advanced adaptive control method that reduces the level of uncer- 
tainty in the system modeling error and improves the overall control authority, by precise modeling techniques. The motivation to use adaptive approximation based control methodology is founded on the idea of devising a method that can store the model information as a function of the operating point and be able to recall the model information as needed to maintain the desired system performance during changes in the operating point [19].

Consider again the 1-dimensional state SISO system representation as

$$
\begin{aligned}
\dot{x}(t) & =f(x(t))+g(x(t)) u(t), \\
y(t) & =x(t),
\end{aligned}
$$

except here we assume that $f(x(t))=\theta_{f}^{T} \phi_{f}(x)$ and $g(x(t))=\theta_{g}^{T} \phi_{g}(x)$ are unknown functions with unknown parameter vectors $\theta_{f}$ and $\theta_{g}$ that require online estimation. The vector of basis functions $\phi_{f}(x)$ and $\phi_{g}(x)$ are determined during the offline design phase, in our case we will be using the spline basis functions. Therefore, we define the spline network approximator functions $\hat{f}(x)=\hat{\theta}_{f}^{T} \phi_{f}(x)$ and $\hat{g}(x)=\hat{\theta}_{g}^{T} \phi_{g}(x)$, where $\hat{\theta}_{f}$ and $\hat{\theta}_{g}$ will be updated via adaptive laws with proper consideration taken to initialize the parameter estimates. A simple choice for an adaptive feedback linearizing $u(t)$ controller for asymptotic output tracking, yields the control law

$$
u(t)=\frac{1}{\hat{g}(x)}\left(-\lambda e(t)-\hat{f}(x(t))+\dot{y}_{d}(t)\right)
$$

where the tracking error is $e(t)=y_{d}(t)-y(t)$, and when applied to the system $(2.7)$, results in the closed-loop tracking error dynamics as

$$
\begin{aligned}
\dot{e}(t) & =\dot{y}_{d}(t)-\dot{y}(t), \\
& =\dot{y}_{d}(t)-f(x)-g(x) u(t) .
\end{aligned}
$$

Rearranging the equation to be in terms of the parameter estimation errors $\tilde{\theta}_{f}(t)=$ $\theta_{f}-\hat{\theta}_{f}(t)$, and $\tilde{\theta}_{g}(t)=\theta_{g}-\hat{\theta}_{g}(t)$, yields the following form for the closed-loop tracking error dynamics

$$
\dot{e}(t)=-\lambda e(t)+\tilde{\theta}_{f}^{T} \phi_{f}(x)+\tilde{\theta}_{g}^{T} \phi_{g}(x) u \text {. }
$$


Now that we have defined the error dynamics we can develop our adaptive update laws by considering the Lyapunov positive definite function candidate

$$
V\left(e, \tilde{\theta}_{f}, \tilde{\theta}_{g}\right)=\frac{1}{2}\left(e^{2}+\tilde{\theta}_{f}^{T} \Gamma_{f}^{-1} \tilde{\theta}_{f}+\tilde{\theta}_{g}^{T} \Gamma_{g}^{-1} \tilde{\theta}_{g}\right)
$$

as a measure of the system's error functions $e(t), \tilde{\theta}_{f}(t)$ and $\tilde{\theta}_{g}(t)$. The time derivative of $V$ along the corresponding solutions is

$$
\dot{V}=e \dot{e}+\tilde{\theta}_{f}^{T} \Gamma_{f}^{-1} \dot{\tilde{\theta}}_{f}+\tilde{\theta}_{g}^{T} \Gamma_{g}^{-1} \dot{\tilde{\theta}}_{g}
$$

using the fact that $\dot{\tilde{\theta}}_{f}(t)=-\dot{\hat{\theta}}_{f}(t), \dot{\tilde{\theta}}_{g}(t)=-\dot{\hat{\theta}}_{g}(t)$, and the tracking error dynamics defined in (2.10), the time derivative of $V$ becomes

$$
\dot{V}=e\left(-\lambda e(t)+\tilde{\theta}_{f}^{T} \phi_{f}(x)+\tilde{\theta}_{g}^{T} \phi_{g}(x) u\right)+\tilde{\theta}_{f}^{T} \Gamma_{f}^{-1} \dot{\hat{\theta}}_{f}+\tilde{\theta}_{g}^{T} \Gamma_{g}^{-1} \dot{\hat{\theta}}_{g}
$$

Combining like terms to solve for $\dot{\hat{\theta}}_{f}$ and $\dot{\hat{\theta}}_{g}$, results in

$$
\dot{V}=-\lambda e^{2}(t)+\tilde{\theta}_{f}^{T} \Gamma_{f}^{-1}\left(\Gamma_{f} e \phi_{f}(x)+\dot{\hat{\theta}}_{f}\right)+\Gamma_{g}^{-1}\left(\Gamma_{g} e \phi_{g}(x) u+\dot{\hat{\theta}}_{g}\right)
$$

Choosing the adaptive update laws to ensure that $\dot{V} \leq 0$, gives us

$$
\begin{aligned}
& \dot{\hat{\theta}}_{f}(t)=-\Gamma_{f} e(t) \phi_{f}(x), \\
& \dot{\hat{\theta}}_{g}(t)=-\Gamma_{g} u(t) e(t) \phi_{g}(x),
\end{aligned}
$$

which simplifies (2.14) to be

$$
\dot{V}=-\lambda e^{2}(t) \leq 0
$$

This ensures the system stability and desired tracking performance. Although this example is for the simplest case with only one state-variable, a similar development can be expanded to include multiple state variable designs with multiple inputs and outputs. In the next two subsections we briefly discuss the control of aircraft with synthetic jet actuators and spacecraft with fuel slosh, respectively, and present some of the most relevant literature in these important nonlinear system structures. 


\subsection{Problem Formulation}

There are two main problems that we will address in this dissertation that are of importance to advanced spacecraft and aircraft systems. The first problem is found in aircraft systems with actuator nonlinearities, a new type of aircraft control device known as synthetic jet actuators have such an inherent nonlinearity. The other problem deals with nonlinear dynamical systems that fail to meet the requirements for backstepping and/or feedback linearization, one such a system is spacecraft with fuel slosh dynamics included rendering a nonminimum phase system model. In this brief subsection we will introduce the basic problem formulation for these two challenges.

Synthetic Jet Actuator Nonlinearities of Aircraft Systems. To begin the formulation of the actuator nonlinearity compensation problem, consider a linear time-invariant plant to represent a local linear commercial aircraft model as

$$
\dot{x}(t)=A x(t)+B u(t), y(t)=C x(t), t \geq 0,
$$

where $(A, B)$ is controllable, the angle of attack $\alpha$ is one of the components of the state vector $x(t) \in \Re^{n}, u(t) \in \Re^{m \times 1}$ is the control input, $y(t) \in \Re$ is the output, and $A \in \Re^{n \times n}, B \in \Re^{n \times m}$ and $C \in \Re^{1 \times n}$ are known constant matrices. The actuator nonlinearity is denoted by $N(\cdot)$ :

$$
u(t)=N(v(t))
$$

where $t$ is the time variable, $v(t)$ is physically represented by the input peak-to-peak voltage applied to the synthetic jet actuator's piezoelectric diaphragm which generates the air flow, and $u(t)$ is the equivalent virtual deflection on the airfoil. It has been observed through wind tunnel testing [15] that the synthetic jet actuator nonlinearity characteristic $N(\cdot)$ changes significantly with the varying values of the aircraft's angle of attack, denoted by $\alpha$.

Our challenge is developing a method to design a spline function approximation based adaptive inverse feedback control scheme for an aircraft flight control system 
having synthetic jet actuators. The developed scheme must cancel the actuator nonlinearity $N(\cdot)$, in order to meet the control objective. The basic adaptive inverse state feedback control scheme is that shown in Figure 2.7.

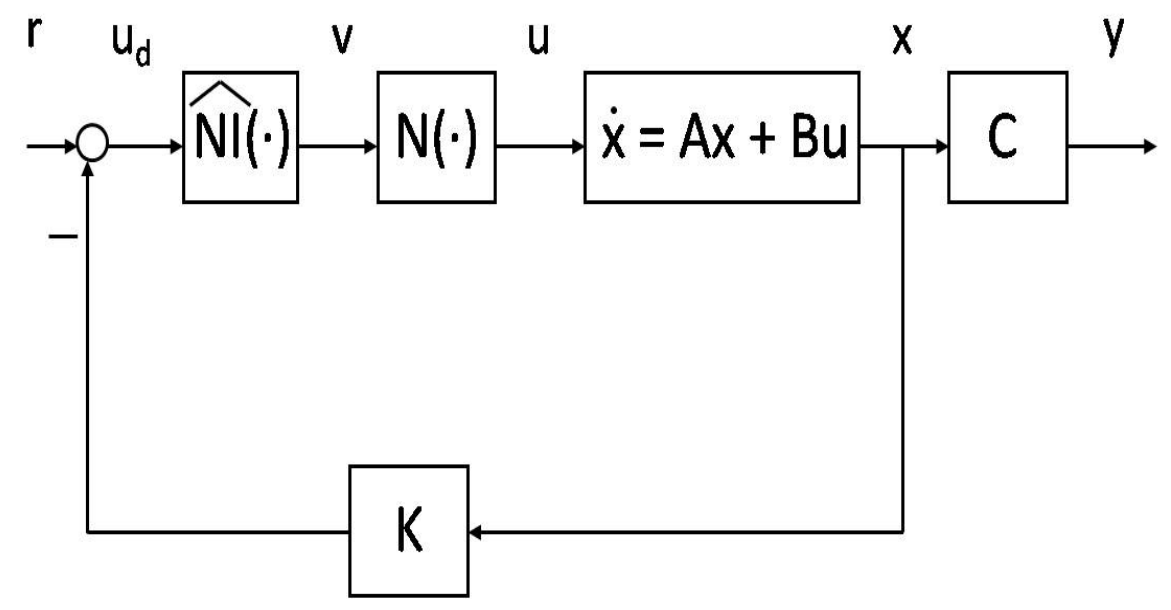

Figure 2.7: Basic adaptive state feedback inverse compensation control scheme.

Dynamic Uncertainties of Nonlinear Systems. Another important problem that we address is the design and analysis of systems that fail to meet the requirements for feedback linearization and other common nonlinear control design methods. These complex nonlinear dynamical systems sometimes have a nonminimum phase structure or have a relative degree that is not well-defined (e.g. ball and beam system, inverted pendulum). To formulate this problem we consider the nonlinear dynamical system model representation as

$$
\begin{aligned}
\dot{x}(t) & =f(x(t))+g(x(t)) u(t), \\
y(t) & =h(x(t))
\end{aligned}
$$

where $x(t) \in \Re^{n}$ is the system state vector, $u(t) \in \Re$ is the control input, $h(x)$ is the output function, $f(x)$ and $g(x)$ are nonlinear functions representing the system dynamics. If $f$ and $g$, are inaccurately modeled or partially unknown, then those 
portions of the functions can be approximated using a spline function network. Next, to separate the unknown portion of each nonlinear function represented by

$$
\dot{x}(t)=\left(f_{o}(x(t))+\bar{f}(x(t))\right)+\left(g_{o}(x(t))+\bar{g}(x(t))\right) u(t)
$$

here the functions $f_{o}(x)$ and $g_{o}(x)$ represent any known components of the system dynamics and $\bar{f}(x)$ and $\bar{g}(x)$ are the unknown parts of the dynamics. The unknown functions of the system model will be approximated over the safe operating compact region $\mathcal{D}$. For any physical system, the region $\mathcal{D}$ is determined at the design stage and is used to ensure the controller maintains the system inside these safe physical limits. An example of such is the design of an electric motor that must operate within specified voltage, current, torque, and speed constraints. If the motor operates outside these safe limits, then it will result in electrical component failure or mechanical part damage that could lead to dangerous life threatening conditions.

To account for the fact that each state of the system in (2.20) may contain unknown functions, we consider rewriting the system dynamics such that the $i^{\text {th }}$ state variable is

$$
\dot{x}_{i}(t)=\left(f_{o_{i}}(x(t))+\bar{f}_{i}(x(t))\right)+\left(g_{o_{i}}(x(t))+\bar{g}_{i}(x(t))\right) u(t),
$$

where $i=1, \ldots, n$, are the number of system state variables defined in the initial model development. Although, this case is only for a single input system it can easily be expanded to the case for systems with multiple inputs.

In order to reduce the modeling error effects on the tracking objectives we consider the unknown parts of the system dynamics the are denoted by the nonlinear functions $\bar{f}(x)$ and $\bar{g}(x)$, these unknown nonlinearities will be approximated by the smooth functions $\hat{f}\left(x, \hat{\theta}_{f}\right)$ and $\hat{g}\left(x, \hat{\theta}_{g}\right)$, respectively. The vectors $\hat{\theta}_{f} \in \Re^{q_{f}}$ and $\hat{\theta}_{g} \in \Re^{q_{g}}$ represent the adjustable parameters for each approximating function, where $\theta_{f}^{*}$ and $\theta_{g}^{*}$ are the corresponding unknown "optimal" parameter (weight) vectors. The nonlinear set of system equations (2.21) are expanded to be expressed for the $i^{\text {th }}$ state we develop a linearly parametrized spline function approximation for each of the functions and 
denote them as $f_{i}^{*}\left(x, \theta_{f_{i}}^{*}\right), g_{i}^{*}\left(x, \theta_{g_{i}}^{*}\right)$. That is, the nonlinear system functions can be approximated by multivariable B-splines defined as

$$
\bar{f}_{i}\left(x, \theta_{f_{i}}\right) \triangleq f_{i}^{*}\left(x, \theta_{f_{i}}^{*}\right) \approx \theta_{f_{i}}^{* T} B_{f_{i}}(x)+\eta_{f_{i}}(x)
$$

and

$$
\bar{g}_{i}\left(x, \theta_{g_{i}}\right) \triangleq g_{i}^{*}\left(x, \theta_{g_{i}}^{*}\right) \approx \theta_{g_{i}}^{* T} B_{g_{i}}(x)+\eta_{g_{i}}(x)
$$

Where the expressions are,

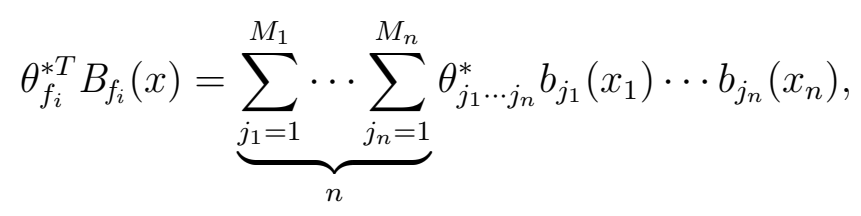

and

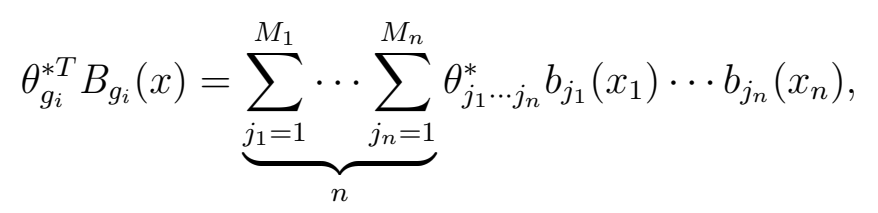

$\eta_{f_{i}}, \eta_{g_{i}}$, represent the $i^{t h}$ spline function approximation errors and for B-splines they are bounded by positive constants $\left\|\eta_{f_{i}}\right\| \leq \eta_{f f_{i}},\left\|\eta_{g_{i}}\right\| \leq \eta_{g g_{i}}$, and approach zero in a cubic fashion. $\theta_{j_{1} \cdots j_{n}}^{*}$ for each $i^{\text {th }}$ approximator is one of $\left(M_{1} \cdot M_{2} \cdots M_{n}\right)$ unknown B-spline coefficients, and $b_{j_{1}}\left(x_{1}\right), \cdots, b_{j_{n}}\left(x_{n}\right)$, are the $n$ univariable B-spline basis elements, and the estimator for each $i^{\text {th }}$ nonlinear approximation model function as

$$
\hat{f}_{i}(x)=\hat{f}_{i}\left(x ; \hat{\theta}_{f_{i}}\right)=\hat{\theta}_{f_{i}}^{T} B_{f_{i}}(x)
$$

and

$$
\hat{g}_{i}(x)=\hat{g}_{i}\left(x ; \hat{\theta}_{g_{i}}\right)=\hat{\theta}_{g_{i}}^{T} B_{g_{i}}(x)
$$

The inherent $i^{t h}$ spline-function approximation errors as $\eta_{f_{i}}(x), \eta_{g_{i}}(x)$ are

$$
\begin{aligned}
& \eta_{f_{i}}(x)=f_{i}\left(x, \theta_{f_{i}}\right)-f_{i}^{*}\left(x, \theta_{f_{i}}^{*}\right), \\
& \eta_{g_{i}}(x)=g_{i}\left(x, \theta_{g_{i}}\right)-g_{i}^{*}\left(x, \theta_{g_{i}}^{*}\right),
\end{aligned}
$$

and the $i^{t h}$ parameter estimation errors $\tilde{\theta}_{f_{i}}(t)=\hat{\theta}_{f_{i}}(t)-\theta_{f_{i}}^{*}$, and $\tilde{\theta}_{g_{i}}(t)=\hat{\theta}_{g_{i}}(t)-\theta_{g_{i}}^{*}$. As the $i^{\text {th }}$ estimates $\hat{\theta}_{f_{i}}^{T}$ and $\hat{\theta}_{g_{i}}^{T}$ approach the desired parameters $\theta_{f_{i}}^{* T}$ and $\theta_{g_{i}}^{* T}$, the 
spline function approximator effectively provides a model for spacecraft with fuel slosh dynamics that can be used for control purposes. Observing the form of these error expressions is critical for adaptive controller design so as to guarantee closed-loop stability. We also note that the above system form does meet the requirement for the popular backstepping control procedure, that is the following condition may not occur $g_{i}\left(x, \theta_{g_{i}}\right) \neq 0$ for any given $x \in D$. Indeed, advanced adaptive control techniques can be developed to handle these residual error terms. In order to implement the spline function network structure, we rewrite the system dynamics represented by (2.22) in terms of the approximating spline functions (2.23) and (2.24), to do this we simply add and subtract the terms $f_{i}^{*}\left(x, \theta_{f_{i}}^{*}\right)$, and $g_{i}^{*}\left(x, \theta_{g_{i}}^{*}\right)$ to the system $(2.22)$. This results in

$$
\begin{aligned}
\dot{x}_{i}(t)= & \left(f_{o_{i}}(x(t))+f_{i}^{*}\left(x, \theta_{f_{i}}^{*}\right)\right)+\left(\bar{f}_{i}(x(t))-f_{i}^{*}\left(x, \theta_{f_{i}}^{*}\right)\right)+\left(g_{o_{i}}(x(t))+g_{i}^{*}\left(x, \theta_{g_{i}}^{*}\right)\right) u(t)+ \\
& +\left(\bar{g}_{i}(x(t))-g_{i}^{*}\left(x, \theta_{g_{i}}^{*}\right)\right) u(t), \\
= & \left(f_{o_{i}}(x(t))+f_{i}^{*}\left(x, \theta_{f_{i}}^{*}\right)\right)+\left(g_{o_{i}}(x(t))+g_{i}^{*}\left(x, \theta_{g_{i}}^{*}\right)\right) u(t)+\eta(x),
\end{aligned}
$$

where the network reconstruction error is defined as

$$
\begin{aligned}
\eta(x) & =\left(\bar{f}_{i}(x)-f_{i}^{*}\left(x, \theta_{f_{i}}^{*}\right)\right)+\left(\bar{g}_{i}(x)-g_{i}^{*}\left(x, \theta_{g_{i}}^{*}\right)\right) u \\
\eta(x) & =\eta_{f_{i}}(x)+\eta_{g_{i}}(x) u
\end{aligned}
$$

From this approximated model we develop our control design to maintain the tracking objective and minimize the system errors using adaptive bounding techniques. In the next section we discuss the steps involved to design such an approximation based adaptive controller.

When we use the terms optimal and minimal they are meant to be in the sense of the infinity norm for the corresponding errors over the region of operation $\mathcal{D}$. The minimum possible deviation between the unknown system functions $f^{*}, g^{*}$ and $\hat{f}\left(x, \hat{\theta}_{f}\right), \hat{g}\left(x, \hat{\theta}_{g}\right)$, respectively, generated from the adaptive approximator, represents the minimal approximation error and is crucial to the controller design. Therefore, it 
is noted that to reduce the minimal approximation error we can increase the number of adjustable weights in the parameter vectors whose size is denoted by $q_{f}$ and $q_{g}$, respectively. Typically, $\theta_{f}^{*}$, and $\theta_{g}^{*}$, are chosen as the values of $\theta_{f}$, and $\theta_{g}$, respectively, that is to minimize the network approximation error uniformly for all $x \in \mathcal{D}$.

It is commonly found that the adaptive update laws $\dot{\hat{\theta}}_{f}$ and $\dot{\hat{\theta}}_{g}$ for the parameter estimate vectors $\hat{\theta}_{f}$ and $\hat{\theta}_{g}$ are modified to incorporate a parameter projection method $\mathcal{P}$ to ensure the parameter estimates remain bounded within a specified region of operation $\mathcal{D}$. The parameter projection modification is also used to maintain the approximation functions stabilizability, such that it does not hold parameters to create stabilizability problems.

In this dissertation we confront the following set of problems:

- Approximation based adaptive compensation of synthetic jet actuators for aircraft flight control;

- Development of a spline function based compensation scheme for systems with actuator nonlinearities;

- Approximation based adaptive control of spacecraft with fuel slosh using spline functions;

- Investigation of other complex nonlinear dynamical challenges using spline functions for approximation based adaptive control. 


\section{Chapter 3}

\section{Modeling of Spacecraft with Fuel Slosh Dynamics}

Planar and rotational control of motion for spacecraft and launch vehicles under going fuel slosh is crucial for rendezvous, launch stage and docking objectives. The degrees of freedom require a higher level of precision for control objectives. In addition, the effect of fuel slosh is pronounced during these complex maneuvers.

\subsection{A Spacecraft Model with Fuel Slosh}

In this section, we formulate the equations of motion for a rigid SISO spacecraft with a spherical fuel tank and include the frequency of the first fuel sloshing mode [9]. To derive these equations using the pendulum analogy, we first need to define a set of generalized coordinates, simply a set of angles and positions that completely describe the motion of the spacecraft and the fuel. We assume that the spacecraft has a partially filled fuel tank and is in a zero gravity environment. The coordinate system used in this design is a two-dimensional body-fixed frame, with the origin and pendulum point of attachment at the center of the tank.

The important variables of the system are the attitude angle $\theta$ of the vehicle, the 
respective axial and transverse acceleration components $a_{x}$ and $a_{z}$, the angle $\psi$ of the approximated pendulum for the fuel. A constant thrust $F_{L}>0$, is assumed to act through the vehicles center of mass along the longitudinal axis, this can also be used as a control input if needed. The input variable used for control is the transverse reaction jet force $F_{T}$. Some other assumed to be uncertain constant variables of the system are the vehicle mass $m$ and moment of inertia $I$ with no fuel, the fuel mass $m_{f}$, and fuel moment of inertia $I_{f}$, the length $a>0$ of the pendulum, and the distance $b$ which is measured on the longitudinal axis from the pendulum fixed point of attachment to the vehicle center of mass location with no fuel. Note that the parameters $m_{f}, I_{f}$, and $a$ depend on the fuel tank shape, the type of fuel, and the fill ratio of the fuel tank [51]. Figure 3.1 shows the spacecraft model with the pendulum approximated fuel slosh mode included.

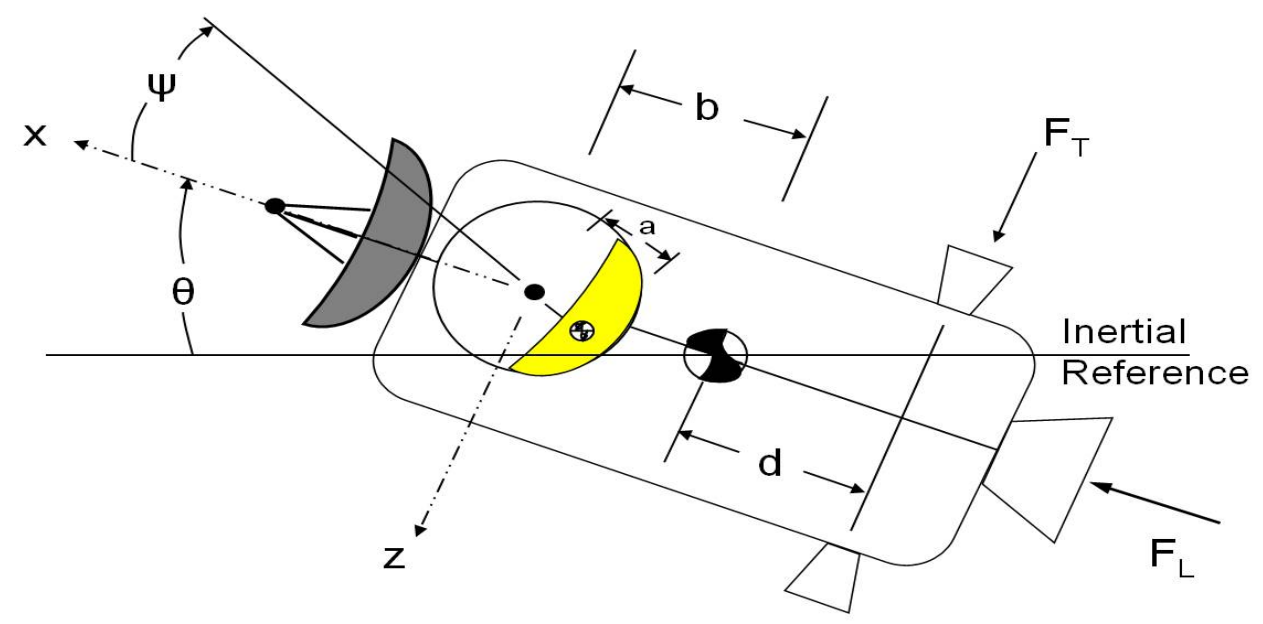

Figure 3.1: Spacecraft with fuel slosh dynamics [9].

The next assumption is that the first fuel sloshing mode is approximated by considering the fuel to be "frozen" into a lump sliding about the sides of the tank. From this assumption the fuel slosh acts like a pendulum with its point of attachment at the center of the tank. In order to develop the equations of motion first consider the free-body diagram for the fuel mass as shown in [64]. 


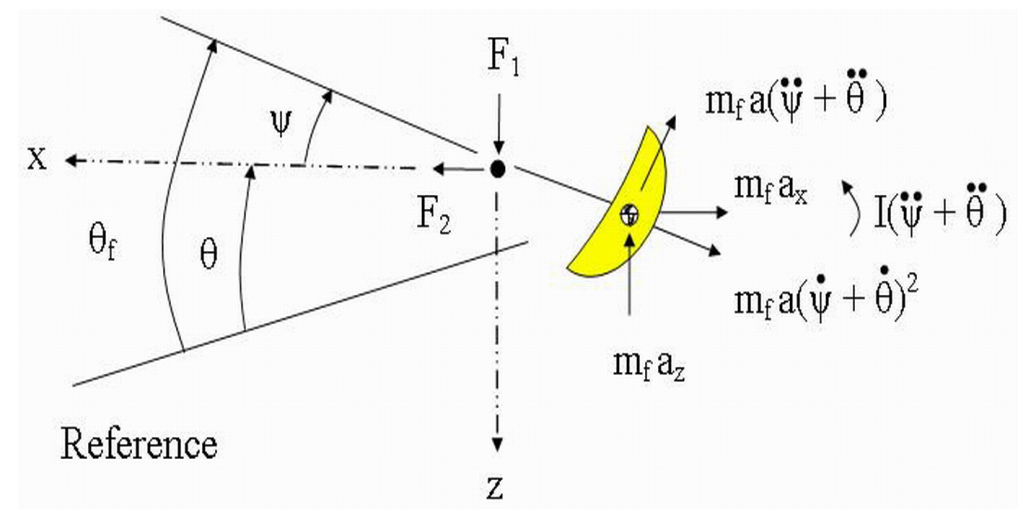

Figure 3.2: Free-body diagram for fuel slosh as pendulum approximation.

Under the declared assumptions first demonstrated in [9], the equations of motion for the fuel are:

$$
\begin{aligned}
& \sum F_{x}=0=F_{2}-m_{f} a_{x}-m_{f} a \dot{\theta}_{f}^{2} \cos \psi-m_{f} a \ddot{\theta}_{f} \sin \psi \\
& \sum F_{z}=0=F_{1}-m_{f} a_{z}+m_{f} a \dot{\theta}_{f}^{2} \sin \psi-m_{f} a \ddot{\theta}_{f} \cos \psi \\
& \sum M_{o}=0=-I_{f} \ddot{\theta}_{f}-m_{f} a^{2} \ddot{\theta}_{f}-m_{f} a a_{x} \sin \psi-m_{f} a a_{z} \cos \psi
\end{aligned}
$$

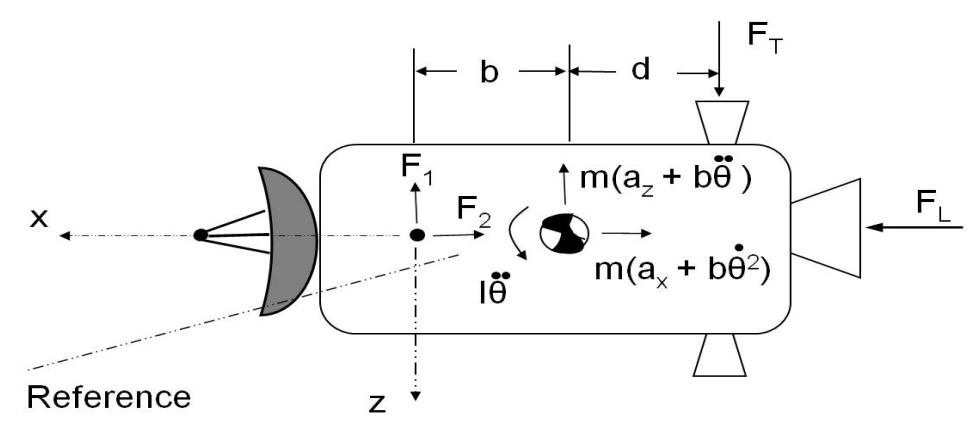

Figure 3.3: Free-body diagram for spacecraft.

From the free-body diagram of just the spacecraft the derivation of the equations of motion for the spacecraft are determined to be: 


$$
\begin{aligned}
\sum F_{x}=0 & =-F_{2}-m\left(a_{x}+b \dot{\theta}^{2}\right)+F_{L}, \\
\sum F_{z}=0 & =-F_{1}-m\left(a_{z}+b \ddot{\theta}\right)+F_{T}, \\
\sum M_{o}=0 & =-I \ddot{\theta}-m b\left(a_{z}+b \ddot{\theta}\right)+(b+d) F_{T} .
\end{aligned}
$$

Although these set of equations might seem challenging at first, with a little effort they can be simplified and combined based on the chosen control objectives. An example of such a combination will now be presented.

In order to combine the equations of motion for the spacecraft and fuel slosh we follow the procedure in [9] and solve the six linear equations for the six unknowns $F_{1}, F_{2}, a_{x}, a_{z}, \ddot{\theta}$, and $\ddot{\theta}_{f}$. Combining the equations to eliminate internal forces and defining the kinematic equations with

$$
\dot{\psi}=\dot{\theta}_{f}-\dot{\theta}
$$

we obtain the equations of motion for both the spacecraft and fuel dynamics. These equations are chosen to represent the coupled motion of the spacecraft and the fuel slosh. This leads to a system that describes the nonlinear equations with the state variables as

$$
x_{1}=\theta, \quad x_{2}=\dot{\theta}, \quad x_{3}=\dot{\theta}_{f}, \quad x_{4}=\psi
$$

These are the state-variables that describe the dynamics of the spacecraft with the fuel slosh included as an internal body. With the state variables defined, we can write the nonlinear equations in state-variable form:

$$
\begin{aligned}
\dot{x} & =f(x, u), \\
y & =h(x),
\end{aligned}
$$


where

$$
x=\left[\begin{array}{c}
\theta \\
\dot{\theta} \\
\dot{\theta}_{f} \\
\psi
\end{array}\right], \quad u=\left[F_{T}\right], \quad y=[\theta] .
$$

This system is governed by the set of equations (3.1)-(3.7) in compact form as:

$$
\left[\begin{array}{c}
\ddot{\theta} \\
\ddot{\theta}_{f}
\end{array}\right]=N^{-1} G .
$$

where

$$
\begin{aligned}
& N=\left[\begin{array}{cc}
I+m k b^{2} & -k m a b \cos x_{4} \\
-k m a b \cos x_{4} & I_{f}+m k a^{2}
\end{array}\right], k=\frac{m_{f}}{m+m_{f}}, \\
& G=\left[\begin{array}{c}
(d+b k) F_{T}-a b k m x_{3}^{2} \sin x_{4} \\
-a k F_{T} \cos x_{4}-a k F_{L} \sin x_{4}+k m a b x_{2}^{2} \sin x_{4}
\end{array}\right] .
\end{aligned}
$$

Now represent the system in state-variable form as:

$$
\begin{array}{llll}
\dot{x}_{1}=\dot{\theta} & = & x_{2}, \\
\dot{x}_{2}=\ddot{\theta} & = & f_{2}(x, u), \\
\dot{x}_{3}=\ddot{\theta}_{f} & = & f_{3}(x, u), \\
\dot{x}_{4}=\dot{\theta}_{f}-\dot{\theta} & = & x_{3}-x_{2},
\end{array}
$$

with

$$
\begin{aligned}
f_{2}(x, u)= & C(x) \cdot\left[\left(m c a b x_{2}\right)^{2} \sin x_{4}-\left(I_{f}+m c a^{2}\right)\left(a b m c x_{3} \sin x_{4}\right)+\right. \\
& \left.+\left[\left(I_{f}+m c a^{2}\right)(d+b c)-m a^{2} c^{2} b \cos ^{2} x_{4}\right] F_{T}-\left(m c^{2} a^{2} \cos x_{4} \sin x_{4}\right) F_{L}\right], \\
f_{3}(x, u)= & C(x) \cdot\left[\left(I+m c b^{2}\right)\left(m c a b x_{2}^{2} \sin x_{4}-\left(m c a b x_{3}\right)^{2} \cos x_{4} \sin x_{4}\right)+\right. \\
& \left.+\left[m c a b \cos x_{4}(d+b c)-\left(I+m c b^{2}\right) a c \cos x_{4}\right] F_{T}-\left[\left(I+m c b^{2}\right) a c \sin x_{4}\right] F_{L}\right],
\end{aligned}
$$


where

$$
\frac{1}{C(x)}=I_{f}\left(I+m c b^{2}\right)+I m c a^{2}+(m c a b)^{2}\left(1-\cos ^{2} x_{4}\right), \quad c=\frac{m_{f}}{m+m_{f}} .
$$

Note that there is a constraint on the equations such that $C(x) \neq 0$, because it appears in the denominator. The above set of equations show the mathematical complexity of a nonlinear spacecraft model with fuel slosh dynamics included. Next, we rewrite the system in the common affine system form to allow for control input design.

$$
\begin{aligned}
& \dot{x}=f(x)+g(x) u, \\
& y=h(x),
\end{aligned}
$$

where we have

$$
f(x)=\left[\begin{array}{c}
x_{2} \\
f_{2}(x) \\
f_{3}(x) \\
x_{3}-x_{2}
\end{array}\right], \quad g(x)=\left[\begin{array}{c}
0 \\
g_{2}(x) \\
g_{3}(x) \\
0
\end{array}\right], \quad u=f
$$

with

$$
\begin{gathered}
f_{2}(x)=C(x) \cdot\left[\left(m c a b x_{2}\right)^{2} \sin x_{4}-\left(I_{f}+m c a^{2}\right)\left(a b m c x_{3} \sin x_{4}\right)-\left(m c^{2} a^{2} \cos x_{4} \sin x_{4}\right) F_{L}\right], \\
f_{3}(x)=C(x) \cdot\left[\left(I+m c b^{2}\right)\left(m c a b x_{2}^{2} \sin x_{4}-\left(m c a b x_{3}\right)^{2} \cos x_{4} \sin x_{4}\right)-\right. \\
\left.-\left(\left(I+m c b^{2}\right) a c \sin x_{4}\right) F_{L}\right] \\
g_{2}(x)=C(x) \cdot\left[\left(I_{f}+m c a^{2}\right)(d+b c)-m a^{2} c^{2} b \cos ^{2} x_{4}\right] \\
g_{3}(x)=C(x) \cdot\left[m c a b \cos x_{4}(d+b c)-\left(I+m c b^{2}\right) a c \cos x_{4}\right],
\end{gathered}
$$

and $h(x)=x_{1}=\theta$ as the system output. Now we solve for the equilibrium states

$$
\dot{x}_{e}=f\left(x_{e}, u_{e}\right)=0 .
$$


This yields the following:

$$
x_{e 1} \in \Re, \quad x_{e 2}=0, \quad x_{e 3}=0, \quad x_{e 4} \in\{0, \pi\} \quad u_{e}=0 .
$$

Next, we determine if the nonlinear system is nonminimum phase. To do this we show that the nonlinear system (3.14) has a relative degree $\rho$ in a region around the point $x_{0}=[0,0,0,0]$. Taking the derivatives of the specified output:

$$
\begin{aligned}
& \dot{y}=\dot{x_{1}}=x_{2}, \\
& \ddot{y}=\dot{x_{2}}=f_{2}(x)+g_{2}(x) u .
\end{aligned}
$$

Hence, the system has relative degree $\rho=2$ in $\Re^{4}$.

The next step is to characterize the zero dynamics by restricting $x$ to the plane

$$
Z^{*}=\left\{x \in \Re^{4} \mid x_{1}=x_{2}=0\right\},
$$

and take $u=u^{*}(x)$ such that $u^{*}(x)$ is the unique solution to the equation

$$
0=\left.f_{2}(x)\right|_{x_{1}=x_{2}=0}+\left.g_{2}(x)\right|_{x_{1}=x_{2}=0} u^{*}(x) .
$$

This yields

$$
u^{*}(x)=-\frac{\left.f_{2}(x)\right|_{x_{1}=x_{2}=0}}{\left.g_{2}(x)\right|_{x_{1}=x_{2}=0}},
$$

Thus the zero dynamics of the system are

$$
\begin{aligned}
& \dot{x_{3}}=f_{z}(x)+g_{z}(x) u^{*}(x), \\
& \dot{x_{4}}=x_{3},
\end{aligned}
$$

where

$$
\begin{gathered}
\left.f_{z}(x)=C(x) \cdot\left[-\left(\operatorname{mcab}_{3}\right)^{2} \cos x_{4} \sin x_{4}\right)-\left(\left(I+m c b^{2}\right) a c \sin x_{4}\right) F_{L}\right], \\
g_{z}(x)=g_{3}(x) .
\end{gathered}
$$


Now we need to determine the asymptotic stability of the equilibrium state $x_{e}=$ $[0,0]$. To be able to conclude whether or not the system is minimum phase. To do this we have plotted the phase portrait shown in Figure 3.4. From the phase portrait we see that the equilibrium state is a center. This zero dynamics system is not asymptotically stable, therefore the nonlinear system for the chosen output of $y(t)=x_{1}(t)$, is nonminimum phase.

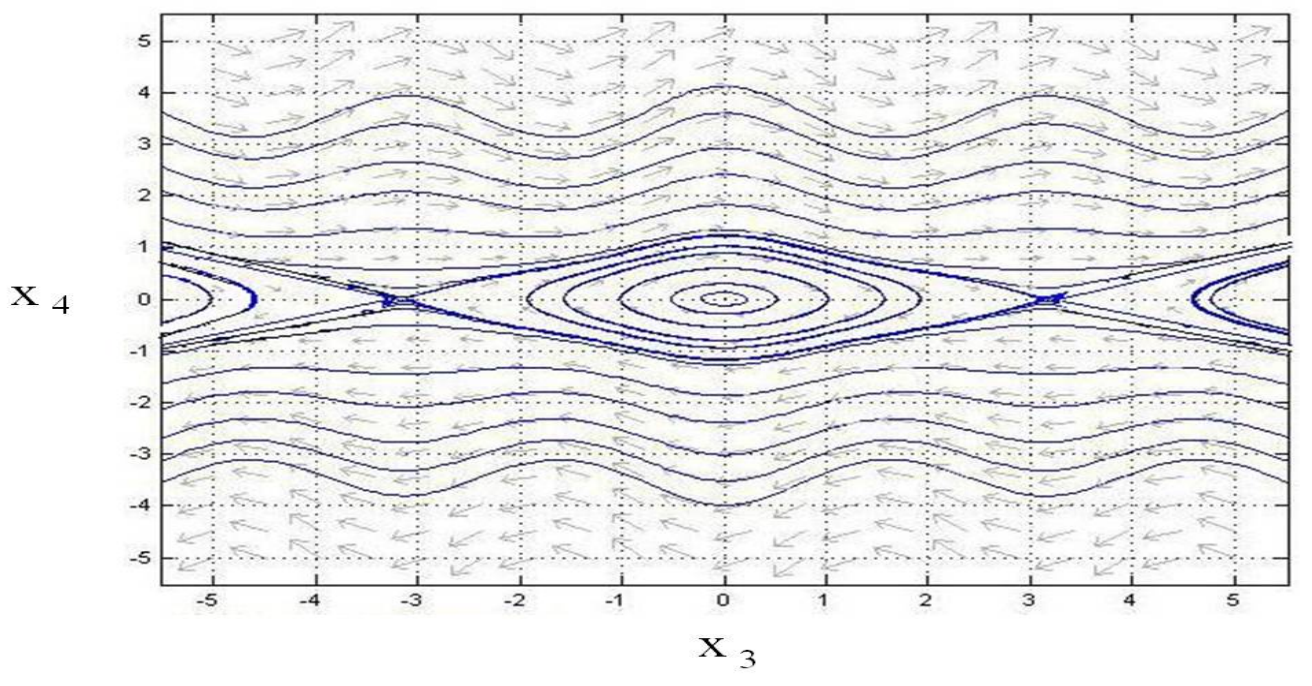

Figure 3.4: Phase portrait $x_{3}-x_{4}$ for the zero dynamics.

\subsection{MIMO Spacecraft with Fuel Slosh}

The model developed in [11] is derived using the Lagrangian formulation to include the transverse, pitch and slosh dynamics into the system model. An interesting aspect of the model is that the equations of motion are derived from the center of the tank and the placement of the center of mass of the spacecraft is in line with the transverse input thrusters. The model has two inputs for control purposes a transverse force $F_{T}$ and a pitching moment $M$. . Let us assume that the accelerating rigid spacecraft moves in a fixed plane, the fuel slosh dynamics are included using the pendulum model shown in Figure 3.5. 


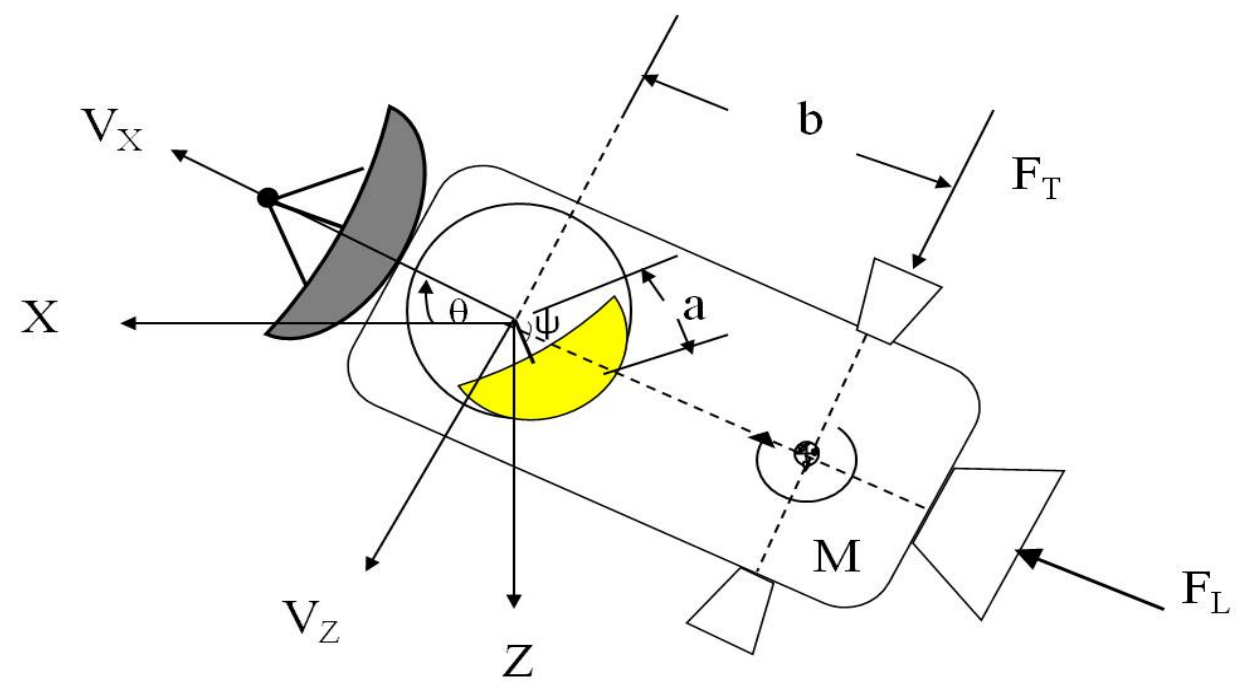

Figure 3.5: Spacecraft with fuel slosh dynamics [51].

Under the declared assumptions the total kinetic energy of the system is

$$
\begin{aligned}
K E= & \frac{1}{2} m\left[v_{x}^{2}+\left(v_{z}+b \dot{\theta}\right)^{2}\right]+\frac{1}{2} I \dot{\theta}^{2}+\frac{1}{2} I_{f}(\dot{\theta}+\dot{\psi})^{2}+ \\
& +\frac{1}{2} m_{f}\left[\left(v_{x}+a(\dot{\theta}+\dot{\psi}) \sin \psi\right)^{2}+\left(v_{z}+a(\dot{\theta}+\dot{\psi}) \cos \psi\right)^{2}\right] .
\end{aligned}
$$

Since the gravitational effects are ignored, the potential energy (PE) is assumed to be zero. Now, we will summarize the Lagrangian-formulation presented in [11] to solve for the equations of motion governing the spacecraft with fuel slosh we write the Lagrangian

$$
L=K E-P E=L(v, \omega, \psi, \dot{\psi}))
$$

First we let the base body translational velocity vector be described by $v \in R^{3}$, and the angular velocity vector by $\omega \in R^{3}$. The internal fuel slosh coordinates are included by $\psi \in R$. The generalized control input forces and moments are defined by $\tau_{t} \in R^{3}$ and $\tau_{r} \in R^{3}$ respectively. To include the internal dissipative forces we assume they 
are derivable from a Rayleigh dissipation function $R$. These variables are defined as

$$
R=\frac{1}{2} \epsilon \dot{\psi}^{2}, v=\left(\begin{array}{c}
v_{x} \\
0 \\
v_{z}
\end{array}\right), \omega=\left(\begin{array}{l}
0 \\
\dot{\theta} \\
0
\end{array}\right), \tau_{t}=\left(\begin{array}{c}
F_{L} \\
0 \\
F_{T}
\end{array}\right), \tau_{r}=\left(\begin{array}{c}
0 \\
M+F_{T} b \\
0
\end{array}\right)
$$

Now, we can write the Lagrange-Euler equations for the spacecraft with internal dynamics by:

$$
\begin{gathered}
\frac{d}{d t} \frac{\partial L}{\partial v}+\hat{\omega} \frac{\partial L}{\partial v}=\tau_{t} \\
\frac{d}{d t} \frac{\partial L}{\partial \omega}+\hat{\omega} \frac{\partial L}{\partial \omega}+\hat{v} \frac{\partial L}{\partial v}=\tau_{r} \\
\frac{d}{d t} \frac{\partial L}{\partial \dot{\psi}}-\frac{\partial L}{\partial \psi}+\frac{\partial R}{\partial \dot{\psi}}=0
\end{gathered}
$$

where $\hat{\omega}$ and $\hat{v}$ represent a skew-symmetric matrix from $\omega=\left(\omega_{1}, \omega_{2}, \omega_{3}\right) \in R^{3}$ :

$$
\hat{\omega}=\left[\begin{array}{ccc}
0 & -\omega_{3} & \omega_{2} \\
\omega_{3} & 0 & -\omega_{1} \\
-\omega_{2} & \omega_{1} & 0
\end{array}\right]
$$

After solving the Lagrange-Euler equations, we have the following equations of motion for the spacecraft with fuel slosh:

$$
\begin{aligned}
F_{L}= & m_{f} a(\ddot{\theta}+\ddot{\psi}) \sin \psi+m b \dot{\theta}^{2}+m_{f} a(\dot{\theta}+\dot{\psi})^{2} \cos \psi+ \\
& +\left(m+m_{f}\right)\left(\dot{v}_{x}+\dot{\theta} v_{z}\right), \\
F_{T}= & \left(m+m_{f}\right)\left(\dot{v}_{z}-\dot{\theta} v_{x}\right)+m_{f} a(\ddot{\theta}+\ddot{\psi}) \cos \psi+m b \ddot{\theta}- \\
& -m_{f} a(\dot{\theta}+\dot{\psi})^{2} \sin \psi, \\
& \\
0= & \left(I_{f}+m_{f} a^{2}\right)(\ddot{\theta}+\ddot{\psi})+m_{f} a\left(\dot{v}_{x}+\dot{\theta} v_{z}\right) \sin \psi+ \\
& +m_{f} a\left(\dot{v}_{z}-\dot{\theta} v_{x}\right) \cos \psi+\epsilon \dot{\psi},
\end{aligned}
$$




$$
M+F_{T} b=\left(I+m b^{2}\right) \ddot{\theta}+m b\left(\dot{v}_{z}-\dot{\theta} v_{x}\right) .
$$

Since the focus is on the effect of fuel slosh during maneuvering, the angle $\theta$ representing the attitude is important to the simulation. This allows $F_{L}$ to primarily control the orbit position and the distance at which the spacecraft travels in the $\mathrm{x}$-direction. The transverse thrusters $F_{T}$ control the movement on the z-axis, while the pitching moment $M$ controls the rotation of the craft in a fixed plane. The nonlinear statevariable form is complex and can be derived by first declaring the states of the system, which in our case will be the position and velocity variables for our spacecraft. To place the system in state-variable form we introduce the following state-variables:

$$
x_{1}=v_{x}, \quad x_{2}=v_{z}, \quad x_{3}=\theta, \quad x_{4}=\dot{\theta}, \quad x_{5}=\psi, \quad x_{6}=\dot{\psi}
$$

With the state-variables defined, we write our nonlinear equations in state-variable form, by plugging in each corresponding state for the chosen variables:

$$
\dot{x}=f(x, u)
$$

where

$$
u=\left[\begin{array}{c}
F_{T} \\
M
\end{array}\right], \quad y=\left[\begin{array}{c}
v_{z} \\
\theta
\end{array}\right] .
$$

The system is governed by the set of equations (3.23)-(3.26), in compact form:

$$
\left[\begin{array}{c}
\dot{v_{x}} \\
\dot{v}_{z} \\
\ddot{\theta} \\
\ddot{\psi}
\end{array}\right]=N^{-1} G_{x}+N^{-1} G_{u} u
$$

where

$$
G_{x}=\left[\begin{array}{c}
F_{L}-m b \dot{\theta}^{2}-\left(m+m_{f}\right) \dot{\theta} v_{z}-m_{f} a(\dot{\theta}+\dot{\psi})^{2} \cos \psi \\
\left(m+m_{f}\right) \dot{\theta} v_{x}+m_{f} a(\dot{\theta}+\dot{\psi})^{2} \sin \psi \\
m b \dot{\theta} v_{x} \\
-\epsilon \dot{\psi}-m_{f} a \dot{\theta} v_{z} \sin \psi+m_{f} a \dot{\theta} v_{x} \cos \psi
\end{array}\right], \quad G_{u}=\left[\begin{array}{cc}
0 & 0 \\
1 & 0 \\
b & 1 \\
0 & 0
\end{array}\right]
$$




$$
N=\left[\begin{array}{cccc}
m+m_{f} & 0 & m_{f} a \sin \psi & m_{f} a \sin \psi \\
0 & m+m_{f} & m_{f} a \cos \psi+m b & m_{f} a \cos \psi \\
0 & m b & I+m b^{2} & 0 \\
m_{f} a \sin \psi & m_{f} a \cos \psi & I_{f}+m_{f} a^{2} & I_{f}+m_{f} a^{2}
\end{array}\right]
$$

After performing some rigorous math (see the Appendix) we obtain the nonlinear system in the control affine form

$$
\begin{aligned}
\dot{x} & =f(x)+g(x) u, \\
y & =h(x),
\end{aligned}
$$

where

$$
f(x)=\left[\begin{array}{c}
f_{1}(x) \\
f_{2}(x) \\
x_{4} \\
f_{4}(x) \\
x_{6} \\
f_{6}(x)
\end{array}\right], g(x)=\left[\begin{array}{cc}
g_{11}(x) & g_{12}(x) \\
g_{21}(x) & g_{22}(x) \\
0 & 0 \\
g_{41}(x) & g_{42}(x) \\
0 & 0 \\
g_{61}(x) & g_{62}(x)
\end{array}\right],
$$

and $h(x)=\left[0, x_{2}, x_{3}, 0,0,0\right]^{T}$ as the system output.

\section{Reduced Order Model}

The full order system is quite complex, therefore in this Dissertation we will study a realistic reduced order system with the logical assumption that the pitch and slosh dynamics have negligible influence on the axial acceleration [11]. First we rewrite $(3.23)$ as

$$
\dot{v}_{x}+\dot{\theta} v_{z}=\frac{1}{m+m_{f}}\left[F_{L}-m_{f} a(\ddot{\theta}+\ddot{\psi}) \sin \psi-m b \dot{\theta}^{2}-m_{f} a(\dot{\theta}+\dot{\psi})^{2} \cos \psi\right] .
$$

The stated assumption allows us to simplify this to

$$
\dot{v}_{x}+\dot{\theta} v_{z}=\frac{F_{L}}{m+m_{f}}
$$


plugging this into (3.25), we obtain the following reduced order model

$$
\begin{gathered}
F_{T}=\left(m+m_{f}\right)\left(\dot{v}_{z}-\dot{\theta} v_{x}\right)+m_{f} a(\ddot{\theta}+\ddot{\psi}) \cos \psi+m b \ddot{\theta}-m_{f} a(\dot{\theta}+\dot{\psi})^{2} \sin \psi \\
0=\left(I_{f}+m_{f} a^{2}\right)(\ddot{\theta}+\ddot{\psi})+m_{f} a\left(\frac{F_{L}}{m+m_{f}}\right) \sin \psi+ \\
+m_{f} a\left(\dot{v}_{z}-\dot{\theta} v_{x}\right) \cos \psi+\epsilon \dot{\psi} \\
M+F_{T} b=\left(I+m b^{2}\right) \ddot{\theta}+m b\left(\dot{v}_{z}-\dot{\theta} v_{x}\right)
\end{gathered}
$$

Now $v_{x}(t)$ is considered as an exogenous input. To place the reduced order system in state-variable form we define:

$$
x_{1}=v_{z}, \quad x_{2}=\theta, \quad x_{3}=\dot{\theta}, \quad x_{4}=\psi, \quad x_{5}=\dot{\psi}
$$

and write out the nonlinear equations (3.31)-(3.33) in state-variable form:

$$
\dot{x}=f(x, u)
$$

where

$$
u=\left[\begin{array}{c}
F_{T} \\
M
\end{array}\right], \quad y=\left[\begin{array}{c}
v_{z} \\
\theta
\end{array}\right] .
$$

In compact form:

$$
\left[\begin{array}{c}
\dot{v}_{z} \\
\ddot{\theta} \\
\ddot{\psi}
\end{array}\right]=N^{-1} G_{x}+N^{-1} G_{u} u
$$

where

$$
\begin{aligned}
& G_{x}=\left[\begin{array}{c}
\left(m+m_{f}\right) \dot{\theta} v_{x}(t)+m_{f} a(\dot{\theta}+\dot{\psi})^{2} \sin \psi \\
m b \dot{\theta} v_{x}(t) \\
-\epsilon \dot{\psi}-\frac{m_{f} a F_{L}}{m+m_{f}} \sin \psi+m_{f} a \dot{\theta} v_{x}(t) \cos \psi
\end{array}\right], \quad G_{u}=\left[\begin{array}{cc}
1 & 0 \\
b & 1 \\
0 & 0
\end{array}\right], \\
& N=\left[\begin{array}{ccc}
m+m_{f} & m_{f} a \cos \psi+m b & m_{f} a \cos \psi \\
m b & I+m b^{2} & 0 \\
m_{f} a \cos \psi & I_{f}+m_{f} a^{2} & I_{f}+m_{f} a^{2}
\end{array}\right] \text {. }
\end{aligned}
$$


After performing some rigorous math (see the Appendix) we obtain the nonlinear system

$$
\begin{aligned}
& \dot{x}=f(x)+g(x) u, \\
& y=h(x),
\end{aligned}
$$

where

$$
f(x)=\left[\begin{array}{c}
f_{1}(x) \\
x_{3} \\
f_{2}(x) \\
x_{5} \\
f_{3}(x)
\end{array}\right], g(x)=\left[\begin{array}{cc}
g_{11}(x) & g_{12}(x) \\
0 & 0 \\
g_{21}(x) & g_{22}(x) \\
0 & 0 \\
g_{31}(x) & g_{32}(x)
\end{array}\right],
$$

with

$$
\begin{aligned}
& f_{1}(x)=G_{1 x}(x) D n_{1,1}+G_{2 x}(x) D n_{2,1}+G_{3}(x) D n_{3,1}, \\
& f_{2}(x)=G_{1 x}(x) D n_{1,2}+G_{2 x}(x) D n_{2,2}+G_{3}(x) D n_{3,2}, \\
& f_{3}(x)=G_{1 x}(x) D n_{1,3}+G_{2 x}(x) D n_{2,3}+G_{3}(x) D n_{3,3}, \\
& g_{11}(x)=G_{1 u} D n_{1,1}+G_{2 u 1} D n_{2,1}, \quad g_{12}(x)=G_{2 u 2} D n_{2,1}, \\
& g_{21}(x)=G_{1 u} D n_{1,2}+G_{2 u 1} D n_{2,2}, \quad g_{22}(x)=G_{2 u 2} D n_{2,2}, \\
& g_{31}(x)=G_{1 u} D n_{1,3}+G_{2 u 1} D n_{2,3}, \quad g_{32}(x)=G_{2 u 2} D n_{2,3},
\end{aligned}
$$

and $h(x)=\left[x_{1}, x_{2}, 0,0,0\right]^{T}$ as the system output. Now we can easily solve for the relative equilibrium states

$x_{e 1}=\frac{F_{L}}{m+m_{f}} t+x_{1}(0), \quad x_{e 2}=0, \quad x_{e 3}=0, \quad x_{e 4}=0, \quad x_{e 5}=0, \quad x_{e 6}=0, \quad u_{e}=[0,0]^{T}$.

\section{Zero Dynamics Analysis}

Next we determine if the MIMO nonlinear system is minimum phase. Applying the definition given in [28], we first determine the relative degree $\left\{r_{1}, r_{2}\right\}$ of the system by differentiating the outputs until we have a component of the input explicitly 
appearing. Taking the derivatives of the specified outputs:

$$
\begin{aligned}
& y_{1}=x_{2}, \\
& \dot{y_{1}}=\dot{x_{2}}=f_{2}(x)+g_{21}(x) u_{1}+g_{22}(x) u_{2}, \\
& y_{2}=x_{3}, \\
& \dot{y_{2}}=\dot{x_{3}}=x_{4}, \\
& \ddot{y_{2}}=\dot{x_{4}}=f_{4}(x)+g_{41}(x) u_{1}+g_{42}(x) u_{2} .
\end{aligned}
$$

Hence, the system has relative degree $\left\{r_{1}=1, r_{2}=2\right\}$ in $\Re^{6}$. The next step is to characterize the zero dynamics by restricting $x$ to

$$
Z^{*}=\left\{x \in \Re^{6} \mid h_{1}(x)=h_{2}(x)=L_{f} h_{2}(x)=0\right\},
$$

where the Lie derivatives are

$$
\begin{aligned}
L_{f} h_{2}(x) & =\frac{\partial h_{2}}{\partial x_{1}} f_{1}+\frac{\partial h_{2}}{\partial x_{2}} f_{2}+\frac{\partial h_{2}}{\partial x_{3}} f_{3}+\frac{\partial h_{2}}{\partial x_{4}} f_{4}+\frac{\partial h_{2}}{\partial x_{5}} f_{5}+\frac{\partial h_{2}}{\partial x_{6}} f_{6}, \\
& =f_{3}(x), \\
& =x_{4} .
\end{aligned}
$$

Therefore,

$$
Z^{*}=\left\{x \in \Re^{6} \mid x_{2}=x_{3}=x_{4}=0\right\}
$$

and take $u=u^{*}(x)$ such that $u^{*}(x)$ is the unique solution to the equations

$$
\begin{aligned}
& 0=\left.f_{2}(x)\right|_{x_{2}=x_{3}=x_{4}=0}+\left.g_{21}(x)\right|_{x_{2}=x_{3}=x_{4}=0} u_{1}^{*}(x)+\left.g_{22}(x)\right|_{x_{2}=x_{3}=x_{4}=0} u_{2}^{*}(x), \\
& 0=\left.f_{4}(x)\right|_{x_{2}=x_{3}=x_{4}=0}+\left.g_{41}(x)\right|_{x_{2}=x_{3}=x_{4}=0} u_{1}^{*}(x)+\left.g_{42}(x)\right|_{x_{2}=x_{3}=x_{4}=0} u_{2}^{*}(x),
\end{aligned}
$$

After solving these two equations for the two unknowns $u_{1}^{*}(x), u_{2}^{*}(x)$, we obtain

$$
\begin{aligned}
& u_{1}^{*}(x)=\left.\frac{g_{22}(x) f_{4}(x)-g_{42}(x) f_{2}(x)}{g_{21}(x) g_{42}(x)-g_{22}(x) g_{41}(x)}\right|_{x_{2}=x_{3}=x_{4}=0}, \\
& u_{2}^{*}(x)=\left.\frac{g_{41}(x) f_{2}(x)-g_{21}(x) f_{4}(x)}{g_{21}(x) g_{42}(x)-g_{22}(x) g_{41}(x)}\right|_{x_{2}=x_{3}=x_{4}=0} .
\end{aligned}
$$


Thus the zero dynamics of the system are

$$
\begin{aligned}
& \dot{x_{1}}=f_{z 1}(x), \\
& \dot{x_{5}}=f_{z 2}(x), \\
& \dot{x_{6}}=f_{z 3}(x),
\end{aligned}
$$

where

$$
\begin{aligned}
f_{z 1}(x) & =\left.f_{1}(x)\right|_{x_{2}=x_{3}=x_{4}=0}+\left.g_{11}(x)\right|_{x_{2}=x_{3}=x_{4}=0} u_{1}^{*}(x)+\left.g_{12}(x)\right|_{x_{2}=x_{3}=x_{4}=0} u_{2}^{*}(x), \\
f_{z 2}(x) & =x_{6}, \\
f_{z 3}(x) & =\left.f_{6}(x)\right|_{x_{2}=x_{3}=x_{4}=0}+\left.g_{61}(x)\right|_{x_{2}=x_{3}=x_{4}=0} u_{1}^{*}(x)+\left.g_{62}(x)\right|_{x_{2}=x_{3}=x_{4}=0} u_{2}^{*}(x) .
\end{aligned}
$$

Now we need to determine the stability of the equilibrium state $x_{z e}=\left[x_{5}=0, x_{6}=0\right]$ of the zero dynamics system (3.37)-(3.39). To be able to conclude whether or not the system is minimum phase. To do this we solve for the Jacobian matrix

$$
J_{z}(x)=\left[\begin{array}{ccc}
\frac{\partial f_{z 1}}{\partial x_{1}} & \frac{\partial f_{z 1}}{\partial x_{5}} & \frac{\partial f_{z 1}}{\partial x_{6}} \\
\frac{\partial f_{z 2}}{\partial x_{1}} & \frac{\partial f_{z 2}}{\partial x_{5}} & \frac{\partial f_{z 2}}{\partial x_{6}} \\
\frac{\partial f_{z 3}}{\partial x_{1}} & \frac{\partial f_{z 3}}{\partial x_{5}} & \frac{\partial f_{z 3}}{\partial x_{6}}
\end{array}\right]
$$

and evaluate this at $x_{z e}$. With the aforementioned physical parameters this yields

$$
J_{z}\left(x_{z e}\right)=\left[\begin{array}{ccc}
0 & 0 & 0 \\
0 & 0 & 1 \\
0 & 0.587 & -0.002417
\end{array}\right] .
$$

Observing the eigenvalues of the matrix $J_{z}\left(x_{z e}\right)$ will give some insight into the stability of the zero dynamics system. We first solve for the eigenvalues $\lambda$ from $\operatorname{det}\left(\lambda I-J_{z}\left(x_{z e}\right)\right)=0:$

$$
\operatorname{det}\left(\lambda I-J_{z}\left(x_{z e}\right)\right)=\operatorname{det}\left[\begin{array}{ccc}
\lambda & 0 & 0 \\
0 & \lambda & -1 \\
0 & -0.587 & \lambda+0.002417
\end{array}\right]=0 .
$$




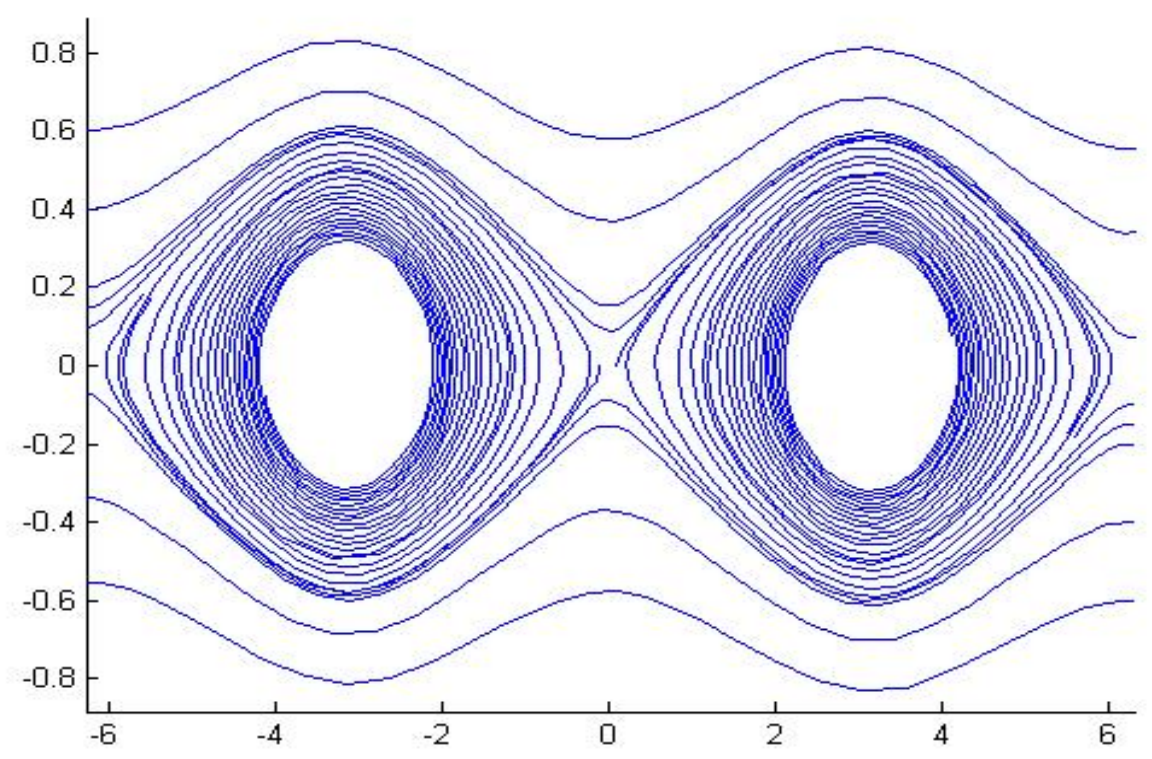

Figure 3.6: Phase portrait $x_{6}-x_{5}$ for the zero dynamics system (3.37)-(3.39).

This yields the eigenvalues: $\lambda_{1}=-0.76741, \lambda_{2}=0.76499$ and $\lambda_{3}=0$. These eigenvalues correspond to a strongly unstable system and what is known as a saddle point in linear stability analysis theory. From this result, we conclude that the original nonlinear system is nonminimum phase. To reinforce our analytical proof we have plotted the phase-portrait shown in Figure 3.6. From the phase portrait we see that the equilibrium state of the origin of the zero dynamics system is not asymptotically stable, therefore the nonlinear system is nonminimum phase.

\section{Reduced Order Model}

$$
\begin{aligned}
\dot{x} & =f(x)+g(x) u, \\
y & =h(x),
\end{aligned}
$$


where

$$
f(x)=\left[\begin{array}{c}
f_{1}(x) \\
x_{3} \\
f_{2}(x) \\
x_{5} \\
f_{3}(x)
\end{array}\right], g(x)=\left[\begin{array}{cc}
g_{11}(x) & g_{12}(x) \\
0 & 0 \\
g_{21}(x) & g_{22}(x) \\
0 & 0 \\
g_{31}(x) & g_{32}(x)
\end{array}\right],
$$

and $h(x)=\left[x_{1}, x_{2}, 0,0,0\right]^{T}$ as the system output. The equilibrium states are

$$
x_{e 1} \in \Re, \quad x_{e 2} \in \Re, \quad x_{e 3}=0, \quad x_{e 4}=0, \pi, \quad x_{e 5}=0, \quad u_{e}=[0,0]^{T} .
$$

Taking the derivatives of the reduced order model specified outputs:

$$
\begin{aligned}
& y_{1}=x_{1}, \\
& \dot{y_{1}}=\dot{x_{1}}=f_{1}(x)+g_{11}(x) u_{1}+g_{12}(x) u_{2}, \\
& y_{2}=x_{2}, \\
& \dot{y_{2}}=\dot{x_{2}}=x_{3}, \\
& \ddot{y_{2}}=\dot{x_{3}}=f_{2}(x)+g_{21}(x) u_{1}+g_{22}(x) u_{2} .
\end{aligned}
$$

Hence, the system has relative degree $\left\{r_{1}=1, r_{2}=2\right\}$ in $\Re^{5}$. The next step is to characterize the zero dynamics by restricting $x$ to

$$
Z^{*}=\left\{x \in \Re^{5} \mid h_{1}(x)=h_{2}(x)=L_{f} h_{2}(x)=0\right\},
$$

where the Lie derivative is

$$
\begin{aligned}
L_{f} h_{2}(x) & =\frac{\partial h_{2}}{\partial x_{1}} f_{1}+\frac{\partial h_{2}}{\partial x_{2}} f_{2}+\frac{\partial h_{2}}{\partial x_{3}} f_{3}+\frac{\partial h_{2}}{\partial x_{4}} f_{4}+\frac{\partial h_{2}}{\partial x_{5}} f_{5}, \\
& =f_{2}(x), \\
& =x_{3} .
\end{aligned}
$$

Therefore,

$$
Z^{*}=\left\{x \in \Re^{5} \mid x_{1}=x_{2}=x_{3}=0\right\},
$$


and take $u=u^{*}(x)$ such that $u^{*}(x)$ is the unique solution to the equations

$$
\begin{aligned}
& 0=\left.f_{1}(x)\right|_{x_{1}=x_{2}=x_{3}=0}+\left.g_{11}(x)\right|_{x_{1}=x_{2}=x_{3}=0} u_{1}^{*}(x)+\left.g_{12}(x)\right|_{x_{1}=x_{2}=x_{3}=0} u_{2}^{*}(x), \\
& 0=\left.f_{2}(x)\right|_{x_{1}=x_{2}=x_{3}=0}+\left.g_{21}(x)\right|_{x_{1}=x_{2}=x_{3}=0} u_{1}^{*}(x)+\left.g_{22}(x)\right|_{x_{1}=x_{2}=x_{3}=0} u_{2}^{*}(x),
\end{aligned}
$$

After solving these two equations for the two unknowns $u_{1}^{*}(x), u_{2}^{*}(x)$, we obtain

$$
\begin{aligned}
& u_{1}^{*}(x)=\left.\frac{g_{12}(x) f_{2}(x)-g_{22}(x) f_{1}(x)}{g_{11}(x) g_{22}(x)-g_{12}(x) g_{21}(x)}\right|_{x_{1}=x_{2}=x_{3}=0}, \\
& u_{2}^{*}(x)=\left.\frac{g_{21}(x) f_{1}(x)-g_{11}(x) f_{2}(x)}{g_{11}(x) g_{22}(x)-g_{12}(x) g_{21}(x)}\right|_{x_{1}=x_{2}=x_{3}=0} .
\end{aligned}
$$

Thus the zero dynamics of the system are

$$
\begin{aligned}
& \dot{x_{4}}=f_{z 1}(x), \\
& \dot{x_{5}}=f_{z 2}(x),
\end{aligned}
$$

where

$$
\begin{aligned}
& f_{z 1}(x)=x_{5}, \\
& f_{z 2}(x)=\left.f_{3}(x)\right|_{x_{1}=x_{2}=x_{3}=0}+\left.g_{31}(x)\right|_{x_{1}=x_{2}=x_{3}=0} u_{1}^{*}(x)+\left.g_{32}(x)\right|_{x_{1}=x_{2}=x_{3}=0} u_{2}^{*}(x) .
\end{aligned}
$$

Now we need to determine the stability of the equilibrium state $x_{z e}=\left[x_{4}=0, x_{5}=0\right]$ of the zero dynamics system (3.40)-(3.41). To be able to conclude whether or not the system is minimum phase. To do this we solve for the Jacobian matrix

$$
J_{z}(x)=\left[\begin{array}{ll}
\frac{\partial f_{z 1}}{\partial x_{4}} & \frac{\partial f_{z 1}}{\partial x_{5}} \\
\frac{\partial f_{z 2}}{\partial x_{4}} & \frac{\partial f_{z 2}}{\partial x_{5}}
\end{array}\right]
$$

and evaluate this at $x_{z e}$. With the aforementioned physical parameters this yields

$$
J_{z}\left(x_{z e}\right)=\left[\begin{array}{cc}
0 & 1 \\
-0.0000 . . & -0.0000 . .
\end{array}\right]
$$

Observing the eigenvalues of the matrix $J_{z}\left(x_{z e}\right)$ will give some insight into the stability of the zero dynamics system. We first solve for the eigenvalues $\lambda$ from $\operatorname{det}(\lambda I-$ $\left.J_{z}\left(x_{z e}\right)\right)=0:$

$$
\operatorname{det}\left(\lambda I-J_{z}\left(x_{z e}\right)\right)=\operatorname{det}\left[\begin{array}{cc}
\lambda & -1 \\
+0.0000 . . & \lambda+0.0000 . .
\end{array}\right]=0 .
$$


This yields the eigenvalues: $\lambda_{1,2}=-1.19 E-20 \pm 2.056 j E-9$. These eigenvalues correspond to a stable system and what is known as a stable focus in linear stability analysis theory. From this result, we conclude that the reduced order nonlinear system is minimum phase.

\subsection{Multi-Mass Fuel Slosh Dynamics}

The spacecraft with fuel slosh models presented thus far in this chapter have only included the first or lowest frequency slosh mode as a single-pendulum. To improve the model accuracy we would have to extend the development of these models to include more frequencies that are higher and are pronounced during complex maneuvering. Recent developments presented in [53], derive a multiple slosh mass-spring model to include higher frequency modes.

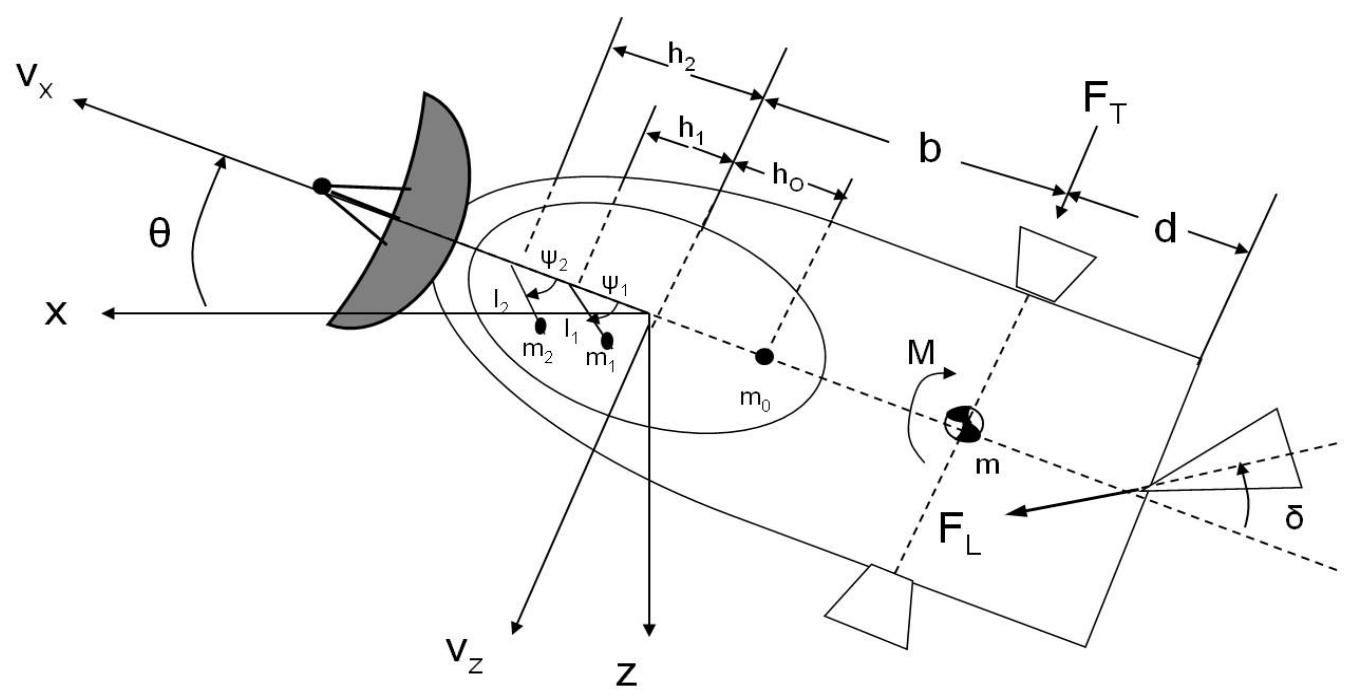

Figure 3.7: A multi-slosh mode pendulum model for a spacecraft with a gimbaled thruster with multiple inputs. [52].

Where $\delta$ denotes the gimbal deflection angle, which is used as one of the additional control inputs. The equations of motion are derived as before from (3.18), but the 
modified total Kinetic Energy (K.E.) is expressed as

$$
\begin{aligned}
K E= & \frac{1}{2} m\left[v_{x}^{2}+\left(v_{z}+b \dot{\theta}\right)^{2}\right]+\frac{1}{2} m_{0}\left[v_{x}^{2}+\left(v_{z}+h_{0} \dot{\theta}\right)^{2}\right]+\frac{1}{2}\left(I+I_{0}\right) \dot{\theta}^{2}+ \\
& +\frac{1}{2} \sum_{i=1}^{N}\left[m _ { i } \left(\left(v_{x}+l_{i}\left(\dot{\theta}+\dot{\psi}_{i}\right) \sin \psi_{i}\right)^{2}+\left(v_{z}-h_{i} \dot{\theta}+l_{i}\left(\dot{\theta}+\dot{\psi}_{i}\right) \cos \psi_{i}\right)^{2}+\right.\right. \\
& \left.+I_{i}\left(\dot{\theta}+\dot{\psi}_{i}\right)^{2}\right]
\end{aligned}
$$

As before we assume the gravitational effects are ignored, and the potential energy (PE) is assumed to be zero. We continue with the modified version of the Lagrangian-formulation presented in [53] to solve for the equations of motion governing the multiple fuel slosh spacecraft with fuel slosh multiple inputs we write the Lagrangian

$$
L=K E-P E=L(v, \omega, \psi, \dot{\psi}))
$$

Recall how we let the base body translational velocity vector be described by $v \in R^{3}$, and the angular velocity vector by $\omega \in R^{3}$. The internal fuel slosh coordinates are included by $\psi \in R$. The generalized control input forces and moments are defined by $\tau_{t} \in R^{3}$ and $\tau_{r} \in R^{3}$ respectively. To include the internal dissipative forces we assume they are derivable from a Rayleigh dissipation function $R$. These variables are defined as

$$
\begin{gathered}
R=\frac{1}{2} \sum_{i=1}^{N} \epsilon_{i} \dot{\psi}_{i}^{2}, v=\left(\begin{array}{c}
v_{x} \\
0 \\
v_{z}
\end{array}\right), \omega=\left(\begin{array}{c}
0 \\
\dot{\theta} \\
0
\end{array}\right), \\
\tau_{t}=\left(\begin{array}{c}
F_{L} \cos \delta \\
0 \\
F_{T}+F_{L} \sin \delta
\end{array}\right), \tau_{r}=\left(\begin{array}{c}
0 \\
M+F_{T} b+F_{L} l \sin \delta \\
0
\end{array}\right) .
\end{gathered}
$$

Next, we solve the Lagrange-Euler equations for the spacecraft with internal dynamics to obtain the modified equations of motion for the multiple slosh mode spacecraft 
model as

$$
\begin{gathered}
F_{L} \cos \delta=\left(m+m_{f}\right) a_{x}+\sum_{i=1}^{N} m_{i} l_{i}\left(\ddot{\theta}+\ddot{\psi}_{i}\right) \sin \psi_{i}+\bar{m} \bar{b} \dot{\theta}^{2}+ \\
+\sum_{i=1}^{N} m_{i} l_{i}\left(\dot{\theta}+\dot{\psi}_{i}\right)^{2} \cos \psi_{i} \\
F_{T}+F_{L} \sin \delta=\left(m+m_{f}\right) a_{z}+\sum_{i=1}^{N} m_{i} l_{i}\left(\ddot{\theta}+\ddot{\psi}_{i}\right) \cos \psi+ \\
+\bar{m} \bar{b} \ddot{\theta}-\sum_{i=1}^{N} m_{i} l_{i}\left(\dot{\theta}+\dot{\psi}_{i}\right)^{2} \sin \psi_{i}, \\
0=\left(I_{i}+m_{i} l_{i}^{2}\right)\left(\ddot{\theta}+\ddot{\psi}_{i}\right)-m_{i} l_{i} h_{i}\left(\ddot{\theta} \cos \psi_{i}+\dot{\theta}^{2} \sin \psi_{i}\right)+ \\
+m_{i} l_{i}\left(a_{x} \sin \psi_{i}+a_{z} \cos \psi_{i}\right)+\epsilon_{i} \dot{\psi}_{i}
\end{gathered}
$$

where $\left(a_{x}, a_{z}\right)=\left(\dot{v}_{x}+\dot{\theta} v_{z}, \dot{v}_{z}-\dot{\theta} v_{x}\right)$ are the axial and transverse acceleration components for the center of the fuel tank, and

$$
\begin{gathered}
\bar{m} \bar{b}=m b-\sum_{i=1}^{N} m_{i} l_{i}, \\
\bar{I}=I+I_{0}+m b^{2}+m_{0} h_{0}^{2}+\sum_{i=1}^{N} m_{i} h_{i}^{2},
\end{gathered}
$$

Although our research does not include an approximation based adaptive control design for this specific model, we can apply our control design from the single slosh model to the multiple slosh model to determine the performance ability. We anticipate that future research will include designs specific for this model to provide for a more accurate mathematical model for the actual spacecraft and liquid fuel included. 


\section{Chapter 4}

\section{Synthetic Jets for Aircraft}

A single synthetic jet consists of an actuator cavity, an oscillating membrane and an orifice (see Figure 4.1). A piezoelectric actuator driven at its resonant frequency functions as the oscillating membrane, and when it oscillates, fluid is alternately expelled and ingested through the orifice. A jet is synthesized by a train of vortices formed at the edge of the orifice.
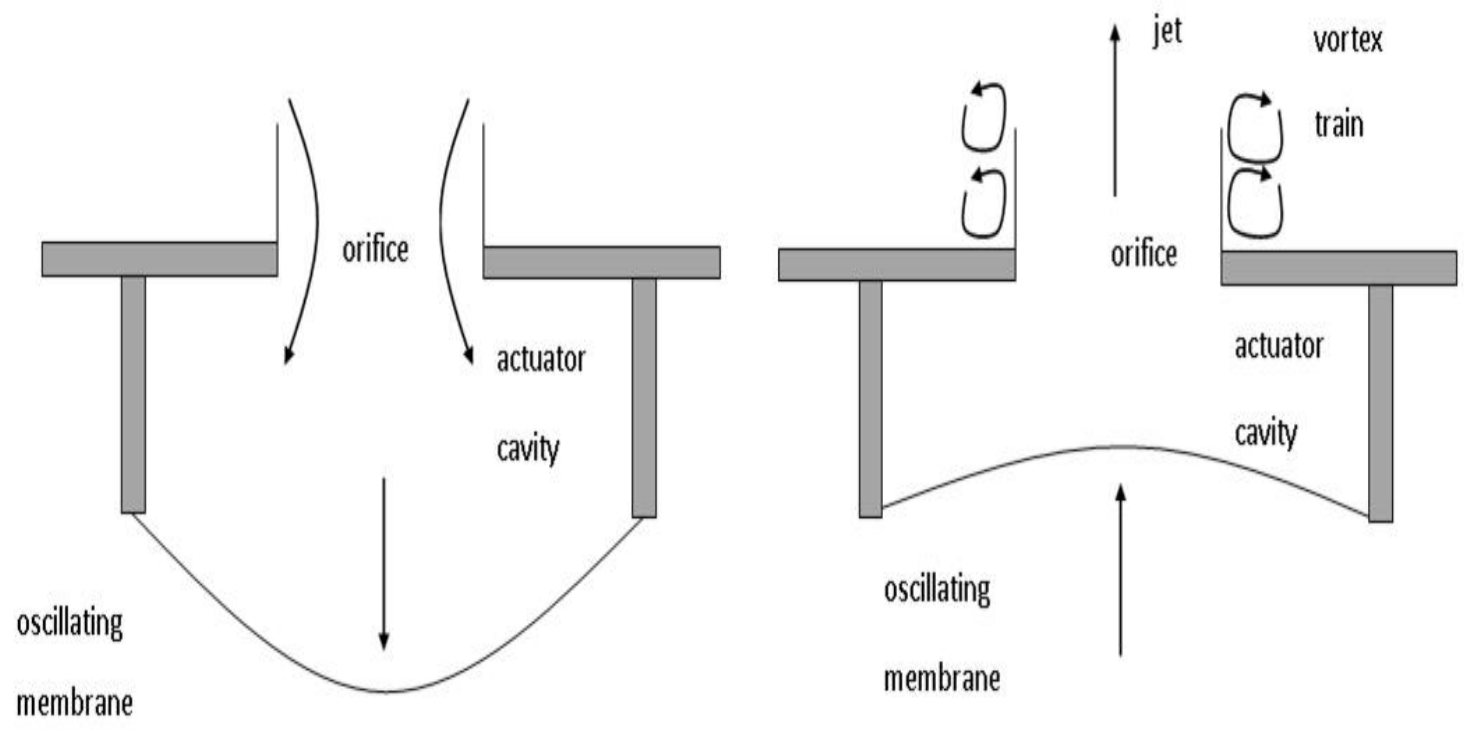

Figure 4.1: Physical structure of a synthetic jet actuator. 


\subsection{Airflow Shaping and Control}

Active flow control using synthetic jet actuators first begin in 1994 [70], since this time researchers have been performing wind tunnel testing to better understand the formation and action of such jets [6]. The primary advantage of these synthetic jets are their zero-net-mass flow nature, which allow the oscillating actuators to synthesize a jet stream without the need for an external source of fluid injection. The generated vortices are formed by the periodic oscillation of the fluid boundary and propagate with a non-zero mean streamwise momentum from the interaction of the newly formed vortices. To better understand this concept we present the side view of an aircraft wing inside of a clean undisturbed airfoil, shown in Figure 4.2.

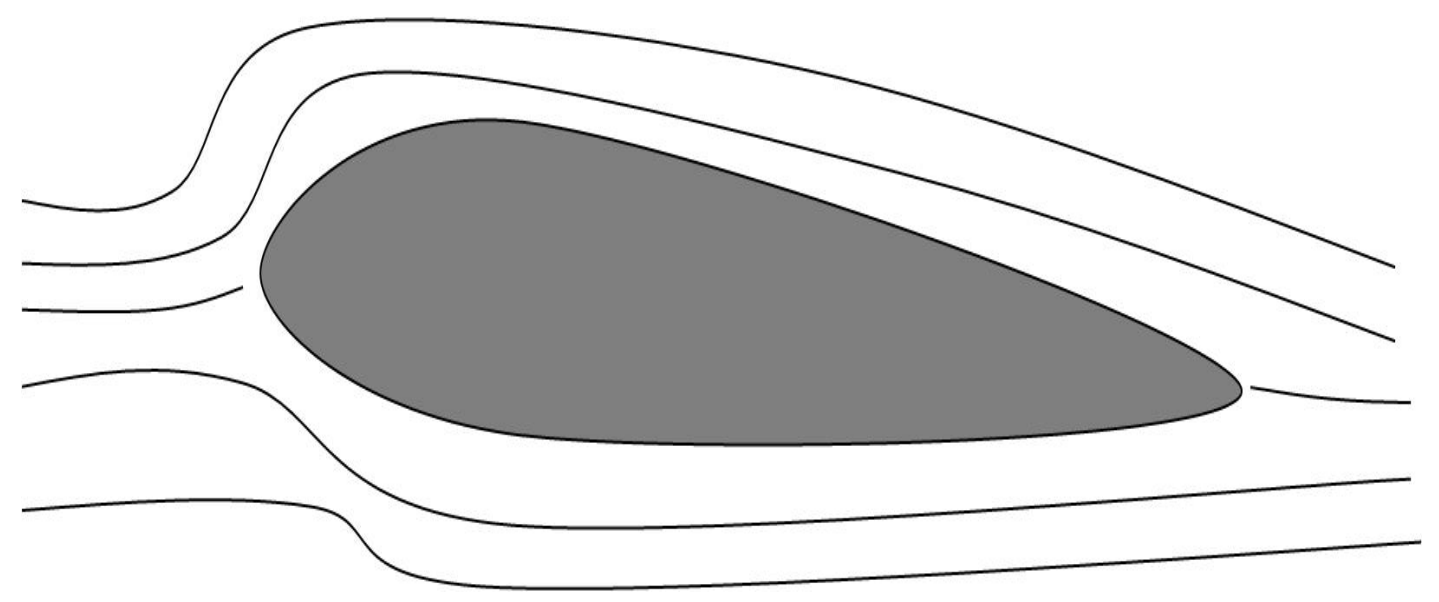

Figure 4.2: Clean airfoil around an aircraft wing.

There are two cases of interest that synthetic jets have been used for in maintaining a clean airfoil across an aircraft's wing: The first case is at low angles of attack and the second is at higher angles of attack. At low angles of attack a separation bubble could form at the surface and disturb the pressure distribution across the clean airfoil resulting in a disturbance shown in Figure 4.3. To counter-act the surface bubble, synthetic jets create a virtual shape change of the airfoil and decrease the surface pressure distribution localized in the area near the synthetic jet actuation ports. 
This provides the needed decrease in pressure drag with only minimal change in lift, to stabilize the airfoil around the aircraft's wing.

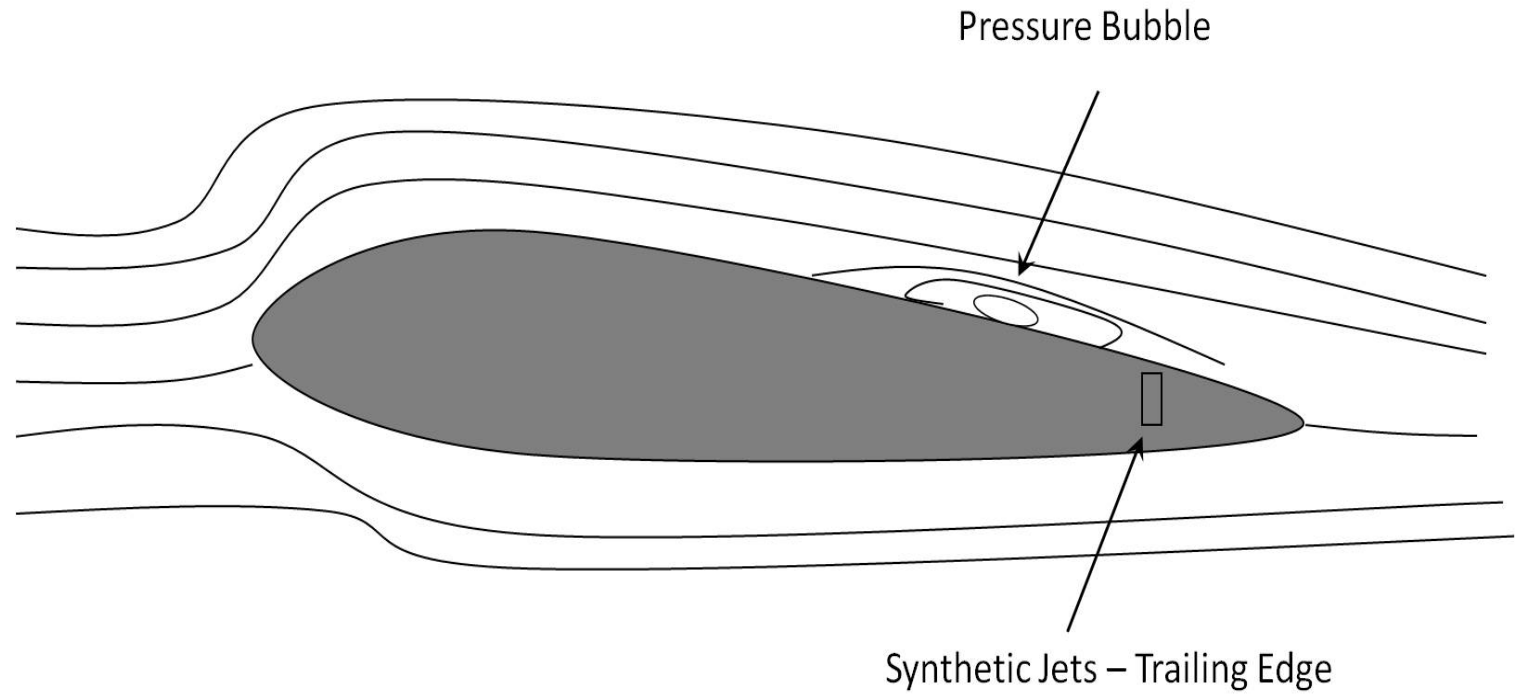

Figure 4.3: Pressure bubble disturbing surface and airfoil around an aircraft wing.

At higher angles of attack the airfoil can experience an adverse pressure gradient region causing the streamlines to separate at the leading edge resulting in excessive drag, lose of lift, and eventually stall. This turbulent flow caused by boundary layer separation near the leading edge of the wing's surface can be overcome by placing synthetic jets at the leading edge of the wing to reattach the airflow and maintain a clean airfoil. The induced fluid vortices created by the synthetic jets reattach the fluid flow pattern and prevent further flow separation. Recent studies $[39,37]$ have shown that placing synthetic jets on the leading edge of an aircraft's wing can delay the onset of stall up to $31^{\circ}$ angle of attack. While synthetic jets placed on the trailing edge of the wing helped generate lift and reduce drag.

As we will see in the next section that the mathematical model used to represent the synthetic jets actuation behavior varies considerably with the change of angle of attack, as discovered by wind tunnel testing data. 


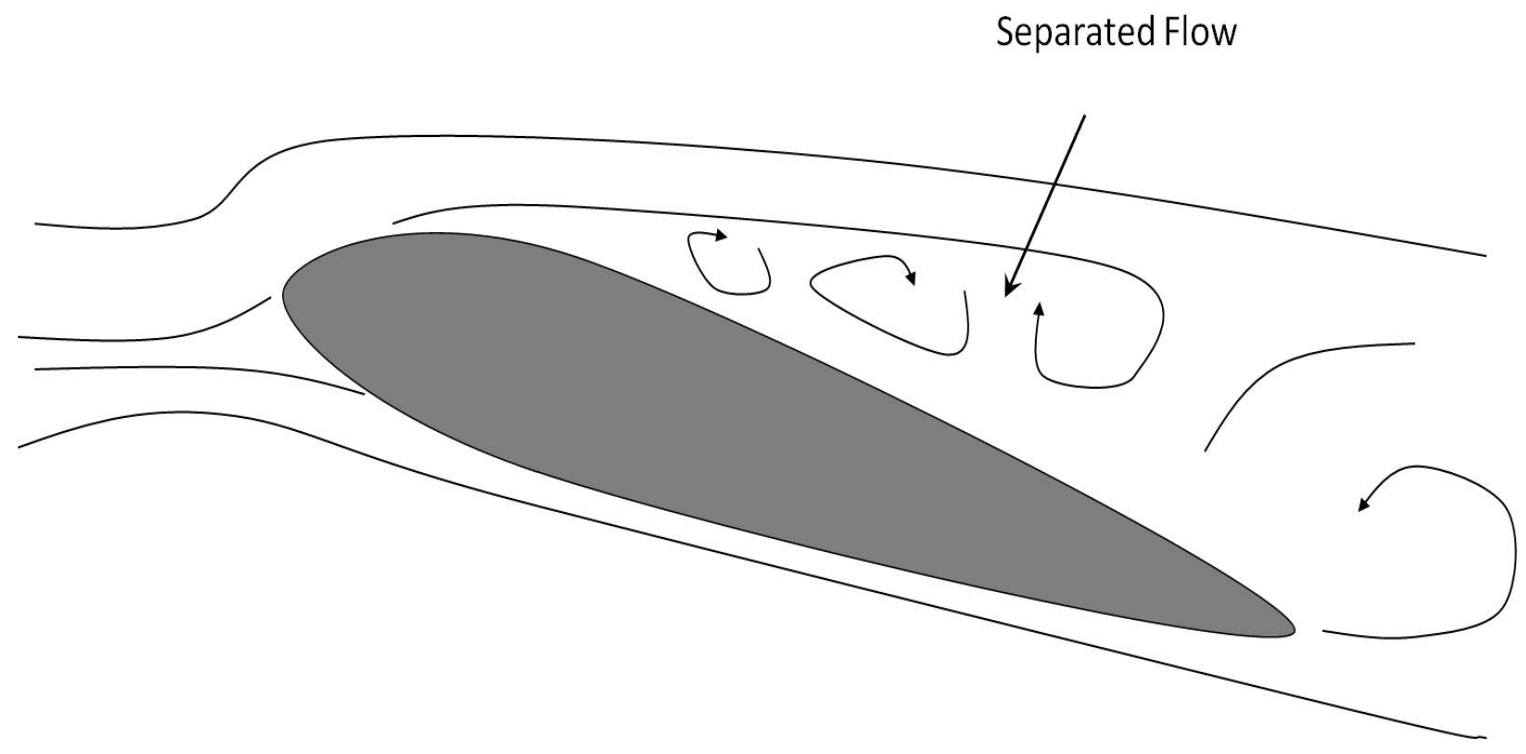

Figure 4.4: Separation of airflow resulting in stall.

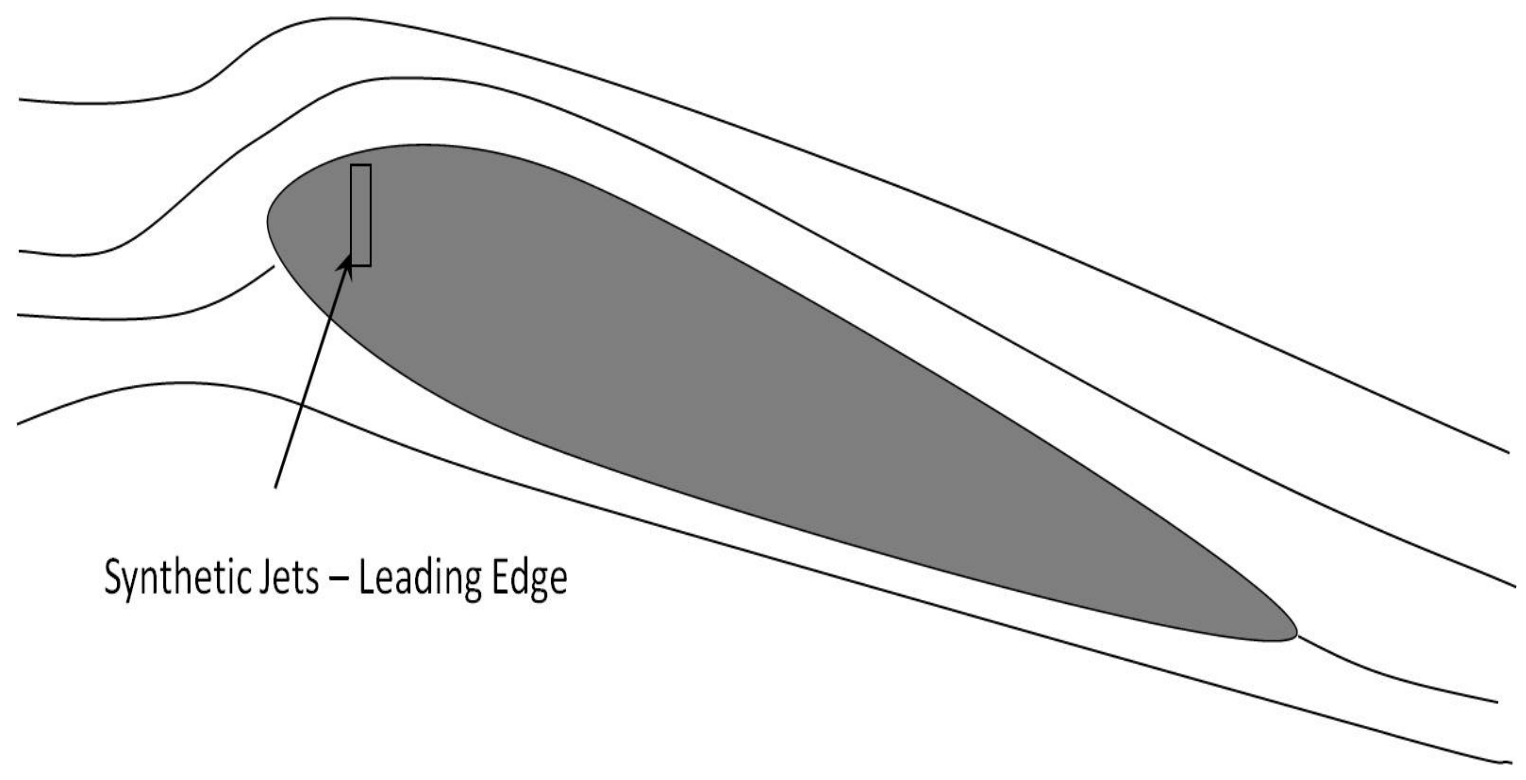

Figure 4.5: Reattached airflow with synthetic jets at the leading edge. 


\subsection{Mathematical Model}

Following the earlier development from Section 2.3 we extend our results to consider each single-input actuator nonlinearity denoted by $N_{i}(\cdot)$ :

$$
u_{i}(t)=N_{i}\left(v_{i}(t)\right)=N_{i}\left(A_{p p_{i}}^{2}(t)\right)
$$

where $i$ is the index of actuator nonlinearity inputs, $t$ is the time variable, $v_{i}(t)=$ $A_{p p_{i}}^{2}(t)$, with $A_{p p_{i}}(t)$ being the $i^{\text {th }}$ input peak-to-peak amplitude voltage applied to each synthetic jet actuator's piezoelectric diaphragm which generates the air flow, and $u_{i}(t)$ is the equivalent virtual deflection on the airfoil. It has been observed through wind tunnel testing [15] that each synthetic jet actuator nonlinearity characteristic $N_{i}(\cdot)$ changes significantly with the varying values of the aircraft's angle of attack, denoted by $\alpha$. As shown in [13], at low angles of attack $\left(\alpha<10^{\circ}\right)$, a parametric model with parameters $\theta_{i}^{*}=\left[\theta_{1_{i}}^{*}, \theta_{2_{i}}^{*}\right]^{T} \in \Re^{2}$ for each actuator nonlinearity characteristic is

$$
u_{i}(t)=N_{i}\left(\theta_{i}^{*} ; v_{i}(t)\right)=f_{i}\left(v_{i}(t)\right)=\theta_{2_{i}}^{*}-\frac{\theta_{1_{i}}^{*}}{v_{i}(t)}
$$

where $v_{i}(t)$ is such that $u_{i}(t) \geq 0$. At higher angles of attack $\left(22^{\circ}<\alpha<24^{\circ}\right)$, the synthetic jet characteristic changes to be nonlinearly parameterized and is represented by

$$
u_{i}(t)=N_{i}\left(\theta_{i}^{*} ; v_{i}(t)\right)=f_{i}\left(v_{i}(t)\right)=\theta_{2_{i}}^{*}+\theta_{1_{i}}^{*} \sin ^{2}\left(\theta_{3_{i}}^{*} v_{i}(t)\right),
$$

for some parameters $\theta_{1_{i}}^{*}, \theta_{2_{i}}^{*}$ and $\theta_{3_{i}}^{*}[15]$.

An adaptive inverse compensation scheme can be easily obtained and used to cancel the effect of the unknown actuator nonlinearities at low angles of attack, that is the function,

$$
v_{i}(t)=\widehat{N I}_{i}\left(\hat{\theta}_{i}(t) ; u_{d_{i}}(t)\right)=\frac{\hat{\theta}_{1_{i}}(t)}{\hat{\theta}_{2_{i}}(t)-u_{d_{i}}(t)}
$$

where $\hat{\theta}_{i}(t)=\left[\hat{\theta}_{1_{i}}, \hat{\theta}_{2_{i}}\right]^{T}$, being the adaptive estimate of $\theta_{i}^{*}$, and $u_{d_{i}}(t)$ is the desired feedback control law to be designed based on the aircraft flight dynamics. An adaptive inverse compensation scheme for the actuator nonlinearity at high angles of attack 
was given in [15]. Combining the two models (4.2) and (4.3), we can see that a synthetic jet characteristic for a wide range of angles of attack is highly nonlinear in nature. Such a nonlinearity may be complicated to describe by an analytical function and it is denoted as $f_{i}\left(v_{i}, \alpha\right)$ whose characteristic depends on $\alpha$.

Our objective is to design a spline function approximation based adaptive inverse feedback control scheme for an aircraft flight control system having synthetic jet actuators. The developed scheme must cancel each actuator nonlinearity $N_{i}(\cdot)$, in order to meet the control objective. As a continuation of our results in [66], we consider an m-input linearized time-invariant plant model of aircraft dynamics with synthetic jet actuators. Such a system that has a controllable state variable form can represent a commercial aircraft model as

$$
\begin{gathered}
\dot{x}(t)=A x(t)+B u(t)=A x(t)+\sum_{i=1}^{m} B_{i} u_{i}, \\
y(t)=C x(t), t \geq 0
\end{gathered}
$$

where the angle of attack $\alpha$ is one of the components of the state vector $x(t) \in \Re^{n}$, $u(t) \in \Re^{m}$ is the control input, $y(t) \in \Re$ is the output, and $A \in \Re^{n \times n}, B \in \Re^{n \times m}$ and $C \in \Re^{1 \times n}$ are known constant parameter matrices. To begin the extension to the multiple input channel nonlinearity case, we define $B_{i}$ as the $i^{\text {th }}$ column of the partitioned system matrix $B$ for $i=1, \ldots, m$, such that our design can access the columns of $B$ separately, corresponding to each $i^{\text {th }}$ input channel that has its own nonlinearity profile associated with it. In addition, we define the control input $u_{i}(t)$ as the $i^{\text {th }}$ element of $u(t)$ that is implemented with the synthetic jet actuators and write the multiple nonlinearity input channels as $u_{i}(t)=N_{i}\left(v_{i}(t)\right)$. Similarly for the inverse structure, $v_{i}(t)=\widehat{N I}_{i}\left(u_{d_{i}}(t)\right)$. This implementation with synthetic jet actuators has a nonlinearity profile given by, $u_{i}(t)=N_{i}\left(v_{i}(t)\right)=f_{i}\left(v_{i}, \alpha\right)$, where $\alpha$ is the angle of attack of the aircraft, and $v_{i}(t)$ is the $i^{t h}$ applied input to the synthetic jet actuators. In other words, $u_{d_{i}}(t)$ is designed as if $u_{i}(t)=u_{d_{i}}(t)$ is true, so it is critical to determine and observe the control error $u_{i}(t)-u_{d_{i}}(t)$. A similar development is 
given in [63] with a neural network framework.

\subsection{Actuator Nonlinearity Characteristic}

From what we have analyzed in the previous section, the synthetic jet actuator characteristic is a general nonlinear function of $v(t)$ and $\alpha$. Therefore, we need to determine a suitable nonlinearity profile for each actuator input nonlinearity for feedback control. In this section, for simplicity we will drop the subscript $i$, such that $f(v, \alpha)$ is used instead of $f_{i}\left(v_{i}, \alpha\right)$, this is equivalent to assuming each $i^{\text {th }}$ nonlinearity has the same profile function. That is,

$$
\begin{array}{r}
u_{i}(t)=f_{i}\left(v_{i}, \alpha\right), \quad i=1,2, \ldots, m, \\
f_{1}=f_{2}=\cdots=f_{m},
\end{array}
$$

although in practice they could be different functions and may even depend on a different set of state variables from each other. One possible realistic choice of the nonlinearity profile $f(v, \alpha)$, based on interpolation of the low and high angle of attack models, is

$$
f(v, \alpha)=a(\alpha) f_{l}(v)+b(\alpha) f_{h}(v)
$$

This candidate nonlinearity profile depends on the applied input to the synthetic jets $v(t)$ and the angle of attack $\alpha$, where $f_{l}(v)$ is the actuator nonlinearity function at a specific low angle $\left(\alpha_{l}=3^{\circ}\right)$ of attack, $f_{h}(v)$ is the nonlinearity function at a high angle $\left(\alpha_{h}=24^{\circ}\right)$ of attack, and, $a(\alpha)$ and $b(\alpha)$ are functions that determine the dependency of the actuator nonlinearity on $\alpha$. In order for this function to be meaningful it must satisfy

$$
a\left(\alpha_{l}\right)=1, a\left(\alpha_{h}\right)=0, b\left(\alpha_{l}\right)=0, b\left(\alpha_{h}\right)=1 .
$$




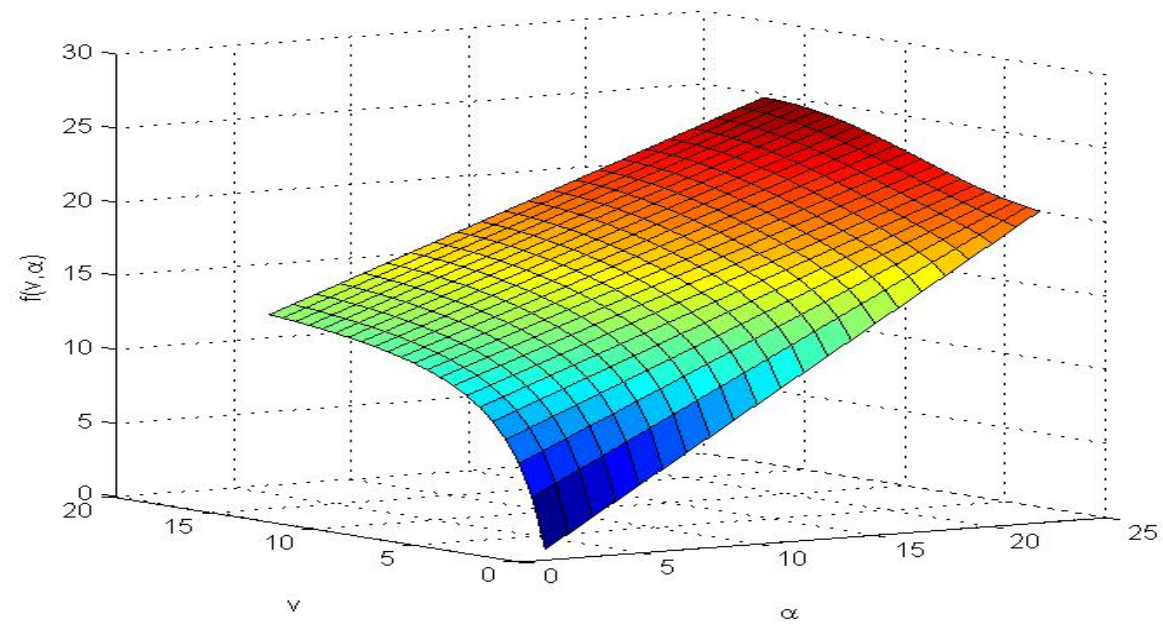

Figure 4.6: Nonlinear profile $f(v, \alpha)$ for varying $\alpha$ and $v$.

That is the nonlinear profile is equal to the actuator nonlinearity functions at the two different angles of attack mentioned above that were determined by experimental data points taken from wind tunnel tests. There are many functions that can be used to represent $a(\alpha)$ and $b(\alpha)$. Given that we have data points for two different angles of attack, the simplest of these are linear functions. With the constraint (4.8), we find

$$
a(\alpha)=-0.0479 \alpha+1.1429, b(\alpha)=0.0479 \alpha-0.1429
$$

As determined in [14], the actuator nonlinearity functions at $\alpha_{l}=3^{\circ}$ and $\alpha_{h}=24^{\circ}$ are given by $f_{l}(v)=15-\frac{33.335}{v}$ and $f_{h}(v)=20+5 \sin ^{2}\left(\pi \frac{v}{32}\right)$, respectively.

As our further wind tunnel tests are performed (as a part of our research project), more information will become available to make the function $f(v, \alpha)$ more precise, as of now it is only valid for $v \in\left[\frac{33.335}{15}, 16\right)$ and $\alpha \in[3,24]$, where the lower bound on $v$ ensures that $f(v, \alpha) \geq 0$. Therefore, this model will be employed in our study for the approximation-based adaptive inverse compensation control design, as the actual model $f(v, \alpha)$ is not currently available. 


\subsection{Other Synthetic Jet Actuator Applications}

Because of the synthetic jets compact size they can easily be used for applications that require an internal flow or some type of turbo machinery [37]. Effective cooling techniques could employ these micro jets to help reduce system overheating which is common in today's laptops. Synthetic jets can augment or even replace cooling fans for such devices as microprocessors, memory chips, batteries and wireless frequency components. Even modern television's that need thermal management of high power LED screens, could use synthetic jets for cooling objectives.

Mixing of fluids could be accomplished with a combination of multiple synthetic jets at precise locations to allow for chemical concentrations to be distributed evenly throughout. A similar technique could be used in the automotive industry to ensure distinct paint colors and evenly coat surfaces of an automobile during the manufacturing stage. Micro-aircraft devices could use these small jets to maneuver and control movement during intelligence gathering missions. Also synthetic jets could provide the right amount of thrust for small underwater vehicles. Although these are only a few of the additional applications synthetic jets can be used for, still much research and development is needed to verify the experimental data to bring them to reality. 


\section{Chapter 5}

\section{Approximation Based Adaptive Control of Spacecraft with Fuel Slosh using Spline Functions}

Recall the nonlinear system defined in (3.14), due to the fact that each state variable may contain unknown functions we follow our development in subsection 2.2 and represent the dynamics of the $i^{\text {th }}$ state variable as

$$
\dot{x}_{i}=\left(f_{o_{i}}(x)+\bar{f}_{i}(x)\right)+\left(g_{o_{i}}(x)+\bar{g}_{i}(x)\right) u,
$$

where $x \in \Re^{4}$ contains the plant states and $i=1,2,3,4$ index the state variables defined in the initial model development, $f_{o_{i}}$ and $g_{o_{i}}$ represent any known information of the dynamic spacecraft model to be approximated. The other functions $\bar{f}_{i}$ and $\bar{g}_{i}$ are the unknown dynamics and will therefore have to be approximated.

\subsection{Approximated SISO System Model}

The system dynamics are therefore 


$$
\begin{aligned}
& \dot{x}_{1}=f_{o_{1}}(x), \\
& \dot{x}_{2}=\bar{f}_{2}(x)+\bar{g}_{2}(x) u, \\
& \dot{x}_{3}=\bar{f}_{3}(x)+\bar{g}_{3}(x) u, \\
& \dot{x}_{4}=f_{o_{4}}(x),
\end{aligned}
$$

this implies that the remaining dynamic functions are $f_{o_{2}}(x)=f_{o_{3}}(x)=g_{o_{1}}(x)=$ $g_{o_{2}}(x)=g_{o_{3}}(x)=g_{o_{4}}(x)=\bar{f}_{1}(x)=\bar{f}_{4}(x)=\bar{g}_{1}(x)=\bar{g}_{4}(x)=0$. Next, we rewrite the system in parametrized form as

$$
\dot{x}_{i}=\left(f_{o_{i}}(x)+\bar{f}_{i}\left(x, \theta_{f_{i}}\right)\right)+\left(g_{o_{i}}(x)+\bar{g}_{i}\left(x, \theta_{g_{i}}\right)\right) u,
$$

where each state in compact form is

$$
\begin{aligned}
& \dot{x}_{1}=f_{o_{1}}(x), \\
& \dot{x}_{2}=\bar{f}_{2}\left(x, \theta_{f_{2}}\right)+\bar{g}_{2}\left(x, \theta_{g_{2}}\right) u, \\
& \dot{x}_{3}=\bar{f}_{3}\left(x, \theta_{f_{3}}\right)+\bar{g}_{3}\left(x, \theta_{g_{3}}\right) u, \\
& \dot{x}_{4}=f_{o_{4}}(x) .
\end{aligned}
$$

The expanded form is

$$
\begin{aligned}
& \dot{x}_{1}=x_{2}, \\
& \dot{x}_{2}=\frac{\theta_{1_{f_{2}}} \phi_{1_{f_{2}}}(x)+\theta_{2_{f_{2}}} \phi_{2_{f_{2}}}(x)+\theta_{3_{f_{2}}} \phi_{3_{f_{2}}}(x)}{\theta_{1_{f_{2}}}+\theta_{5_{f_{2}}}+\theta_{4_{f_{2}}} \phi_{4_{f_{2}}}(x)}+\frac{\theta_{1_{g_{2}}} \phi_{1_{g_{2}}}(x)+\theta_{2_{g_{2}}}}{\theta_{3_{g_{2}}}+\theta_{4_{g_{2}}}+\theta_{5_{g_{2}}} \phi_{2_{g_{2}}}(x)} u, \\
& \dot{x}_{3}=\frac{\theta_{1_{f_{3}}} \phi_{1_{f_{3}}}(x)+\theta_{2_{f_{3}}} \phi_{2_{f_{3}}}(x)+\theta_{3_{f_{3}}} \phi_{3_{f_{3}}}(x)}{\theta_{4_{f_{3}}}+\theta_{5_{f_{3}}}+\theta_{6_{f_{3}}} \phi_{4_{f_{3}}}(x)}+\frac{\theta_{1_{g_{3}}} \phi_{1_{g_{3}}}(x)}{\theta_{2_{g_{3}}}+\theta_{3_{g_{3}}}+\theta_{4_{g_{2}}} \phi_{2_{g_{3}}}(x)} u \\
& \dot{x}_{4}=x_{3}-x_{2} .
\end{aligned}
$$

It is clear that the system is nonlinear in its unknown parameters, therefore, approximation methods must be employed to handle these uncertain nonlinearities. Substituting in the mathematically derived dynamic model equations we obtain the system with the spacecraft physical model parameters 


$$
\begin{array}{ll}
\theta_{1_{f_{2}}}=(m c a b)^{2}, & \theta_{2_{f_{2}}}=-\left(I_{f}+m c a^{2}\right) a b m c, \\
\theta_{3_{f_{2}}}=-m c^{2} a^{2} F, & \theta_{4_{f_{2}}}=-(m c a b)^{2}, \\
\theta_{5_{f_{2}}}=I_{f}\left(I+m c b^{2}\right)+I m c a^{2}, & \theta_{1_{g_{2}}}=-m a^{2} c^{2} b, \\
\theta_{2_{g_{2}}}=\left(I_{f}+m c a^{2}\right)(d+b c), & \theta_{3_{g_{2}}}=(m c a b)^{2}, \\
\theta_{4_{g_{2}}}=I_{f}\left(I+m c b^{2}\right)+I m c a^{2}, & \theta_{5_{g_{2}}}=-(m c a b)^{2}, \\
\theta_{1_{f_{3}}}=\left(I+m c b^{2}\right) m c a b, & \theta_{2_{f_{3}}}=-\left(I+m c b^{2}\right)(m c a b)^{2}, \\
\theta_{3_{f_{3}}}=-\left(I+m c b^{2}\right) a c F, & \theta_{f_{f_{3}}}=(m c a b)^{2}, \\
\theta_{5_{f_{3}}}=I_{f}\left(I+m c b^{2}\right)+I m c a^{2}, & \theta_{6_{f_{3}}}=-(m c a b)^{2}, \\
\theta_{1_{g_{3}}}=m c a b(d+b c)-\left(I+m c b^{2}\right) a c, & \theta_{2_{g_{3}}}=(m c a b)^{2}, \\
\theta_{3_{g_{3}}}=I_{f}\left(I+m c b^{2}\right)+I m c a^{2}, & \theta_{4_{g_{3}}}=-(m c a b)^{2},
\end{array}
$$

and

$$
\begin{array}{lll}
\phi_{1_{f_{2}}}(x)=x_{2}^{2} \sin x_{4}, & \phi_{2_{f_{2}}}(x)=x_{3} \sin x_{4}, & \phi_{3_{f_{2}}}(x)=\cos x_{4} \sin x_{4}, \\
\phi_{4_{f_{2}}}(x)=\cos ^{2} x_{4}, & \phi_{1_{g_{2}}}(x)=\cos ^{2} x_{4}, & \phi_{2_{g_{2}}}(x)=\cos ^{2} x_{4}, \\
\phi_{1_{f_{3}}}(x)=x_{2}^{2} \sin x_{4}, & \phi_{2_{f_{3}}}(x)=x_{3}^{2} \cos x_{4} \sin x_{4}, & \phi_{3_{f_{3}}}(x)=\sin x_{4}, \\
\phi_{1_{g_{3}}}(x)=\cos x_{4}, & \phi_{2_{g_{3}}}(x)=\cos ^{2} x_{4} . &
\end{array}
$$

\subsubsection{Spline Function Approximation Technique}

To overcome these highly nonlinear complexity challenges, for the $i^{\text {th }}$ state we develop a linearly parametrized spline function approximation for each of the uncertain functions $\bar{f}_{i}\left(x, \theta_{f_{i}}\right), \bar{g}_{i}\left(x, \theta_{g_{i}}\right)$, and denote them as $f_{i}^{*}\left(x, \theta_{f_{i}}^{*}\right), g_{i}^{*}\left(x, \theta_{g_{i}}^{*}\right)$, respectively. That is, the uncertain system functions can be approximated by multivariable B-splines defined as

$$
\bar{f}_{i}\left(x, \theta_{f_{i}}\right) \triangleq f_{i}^{*}\left(x, \theta_{f_{i}}^{*}\right) \approx \theta_{f_{i}}^{* T} B_{f_{i}}(x)+\eta_{f_{i}}(x)
$$

and

$$
\bar{g}_{i}\left(x, \theta_{g_{i}}\right) \triangleq g_{i}^{*}\left(x, \theta_{g_{i}}^{*}\right) \approx \theta_{g_{i}}^{* T} B_{g_{i}}(x)+\eta_{g_{i}}(x)
$$


Here the expressions are

$$
\theta_{f_{i}}^{* T} B_{f_{i}}(x)=\sum_{j_{1}=1}^{M_{1}} \cdots \sum_{j_{4}=1}^{M_{4}} \theta_{j_{1} \cdots j_{4}}^{*} b_{j_{1}}\left(x_{1}\right) \cdots b_{j_{4}}\left(x_{4}\right),
$$

and

$$
\theta_{g_{i}}^{* T} B_{g_{i}}(x)=\sum_{j_{1}=1}^{M_{1}} \cdots \sum_{j_{4}=1}^{M_{4}} \theta_{j_{1} \cdots j_{4}}^{*} b_{j_{1}}\left(x_{1}\right) \cdots b_{j_{4}}\left(x_{4}\right),
$$

$\eta_{f_{i}}, \eta_{g_{i}}$, represent the $i^{\text {th }}$ spline function approximation errors and for B-splines they are bounded by known positive constants $\left\|\eta_{f_{i}}\right\| \leq \eta_{f f_{i}},\left\|\eta_{g_{i}}\right\| \leq \eta_{g g_{i}}$, and approach zero in a cubic fashion. $\theta_{j_{1} j_{2} j_{3} j_{4}}^{*}$ for each $i^{t h}$ approximator is one of $\left(M_{1} \cdot M_{2} \cdot M_{3} \cdot M_{4}\right)$ unknown B-spline coefficients, and $b_{j_{1}}\left(x_{1}\right), b_{j_{2}}\left(x_{2}\right), b_{j_{3}}\left(x_{3}\right), b_{j_{4}}\left(x_{4}\right)$ are the univariable B-spline basis elements, and the estimators for each $i^{\text {th }}$ nonlinear approximation model functions are

$$
\hat{f}_{i}(x)=\hat{f}_{i}\left(x ; \hat{\theta}_{f_{i}}\right)=\hat{\theta}_{f_{i}}^{T} B_{f_{i}}(x)
$$

and

$$
\hat{g}_{i}(x)=\hat{g}_{i}\left(x ; \hat{\theta}_{g_{i}}\right)=\hat{\theta}_{g_{i}}^{T} B_{g_{i}}(x) .
$$

Output Tracking Error. Before we move on to discuss the approximationbased adaptive control structure it is imperative for us to observe the inherent errors that exist. Recall the control objective is for the system output signal $y(t)$ to track a desired output $y_{d}(t)$. It is typical to assume that $y_{d}, \dot{y}_{d}, \cdots, y_{d}^{(i)}$ are known and uniformly bounded. This leads us to observe the output tracking error defined as

$$
e(t)=y(t)-y_{d}(t)
$$

Observing the form of the error expressions is critical for adaptive controller design so as to guarantee closed-loop stability. We must also mention that the above system form does not meet the requirement for the popular exact feedback linearization control procedure, that is the system for the chosen output of $y(t)=x_{1}(t)$, has a relative degree of $\rho=2$ which is $<n=4$ for any given $x \in D$. Indeed, advanced adaptive control techniques must be developed to handle these type of systems with a non-minimum phase structure. 
Consider the following change of coordinates similar to that of [49] for neural networks

$$
\begin{aligned}
z_{1}(t) & =x_{1}(t)-y_{d}(t)-\alpha_{1}, \\
z_{2}(t) & =x_{2}(t)-\dot{y}_{d}(t)-\alpha_{2}, \\
z_{3}(t) & =x_{3}(t)-\ddot{y}_{d}(t)-\alpha_{3}, \\
z_{4}(t) & =x_{4}(t)-y_{d}^{(3)}(t)-\alpha_{4},
\end{aligned}
$$

where $\alpha_{i}$ are intermediate designed control functions to be defined in the next section with the stability analysis, for $i=1,2,3,4$. Therefore, the $z$-coordinate dynamics are represented as

$$
\begin{aligned}
& \dot{z}_{1}(t)=\dot{x}_{1}(t)-\dot{y}_{d}(t)-\dot{\alpha}_{1}, \\
& \dot{z}_{2}(t)=\dot{x}_{2}(t)-\ddot{y}_{d}(t)-\dot{\alpha}_{2}, \\
& \dot{z}_{3}(t)=\dot{x}_{3}(t)-y_{d}^{(3)}(t)-\dot{\alpha}_{3}, \\
& \dot{z}_{4}(t)=\dot{x}_{4}(t)-y_{d}^{(4)}(t)-\dot{\alpha}_{4} .
\end{aligned}
$$

Substituting in the system functions from (5.1) we obtain

$$
\begin{aligned}
& \dot{z}_{1}(t)=f_{o_{1}}(x)-\dot{y}_{d}(t)-\dot{\alpha}_{1}, \\
& \dot{z}_{2}(t)=\bar{f}_{2}\left(x, \theta_{f_{2}}\right)+\bar{g}_{2}\left(x, \theta_{g_{2}}\right) u-\ddot{y}_{d}(t)-\dot{\alpha}_{2}, \\
& \dot{z}_{3}(t)=\bar{f}_{3}\left(x, \theta_{f_{3}}\right)+\bar{g}_{3}\left(x, \theta_{g_{3}}\right) u-y_{d}^{(3)}(t)-\dot{\alpha}_{3}, \\
& \dot{z}_{4}(t)=f_{o_{4}}(x)-y_{d}^{(4)}(t)-\dot{\alpha}_{4},
\end{aligned}
$$

and substituting in the coordinates from (5.22) - (5.25) we rewrite the dynamics to be

$$
\begin{aligned}
& \dot{z}_{1}(t)=z_{2}+\alpha_{2}-\dot{\alpha}_{1} \\
& \dot{z}_{2}(t)=z_{3}+\alpha_{3}-x_{3}+\bar{f}_{2}\left(x, \theta_{f_{2}}\right)+\bar{g}_{2}\left(x, \theta_{g_{2}}\right) u-\dot{\alpha}_{2}, \\
& \dot{z}_{3}(t)=z_{4}+\alpha_{4}-x_{4}+\bar{f}_{3}\left(x, \theta_{f_{3}}\right)+\bar{g}_{3}\left(x, \theta_{g_{3}}\right) u-\dot{\alpha}_{3}, \\
& \dot{z}_{4}(t)=z_{3}-z_{2}+\alpha_{3}-\alpha_{2}-\dot{\alpha}_{4}+\ddot{y}_{d}(t)-\dot{y}_{d}(t)-y_{d}^{(4)}(t),
\end{aligned}
$$


where the derivative of the $i^{t h}$ designed control function is defined by

$$
\dot{\alpha}_{i}=\frac{\partial \alpha_{i}}{z_{i}} \dot{z}_{i}+\frac{\partial \alpha_{i}}{\hat{\theta}_{f_{i}}} \dot{\hat{\theta}}_{f_{i}}+\frac{\partial \alpha_{i}}{\hat{\theta}_{g_{i}}} \dot{\hat{\theta}}_{g_{i}}+\frac{\partial \alpha_{i}}{y_{d}{ }^{i-1}} y_{d}^{i}+\frac{\partial \alpha_{i}}{y_{d}{ }^{i}} y_{d}^{i+1}
$$

for $i=1,2,3,4[19]$.

\subsubsection{Stability Analysis}

Now that we have defined the $z$-coordinate dynamics we can develop our adaptive control law, by considering the Lyapunov positive definite function candidate

$$
V\left(z_{i}, \tilde{\theta}_{f_{i}}, \tilde{\theta}_{g_{i}}\right)=\frac{1}{2} \sum_{i=1}^{n}\left(z_{i}^{2}+\tilde{\theta}_{f_{i}}^{T} \Gamma_{f_{i}}^{-1} \tilde{\theta}_{f_{i}}+\tilde{\theta}_{g_{i}}^{T} \Gamma_{g_{i}}^{-1} \tilde{\theta}_{g_{i}}\right)
$$

as a measure of the system error functions $z_{i}(t), \tilde{\theta}_{f_{i}}(t)$, and $\tilde{\theta}_{g_{i}}(t)$, where $n=4$ is the number of system states. Taking the time derivative of $V$ along the corresponding solutions gives

$$
\dot{V}=\sum_{i=1}^{n}\left(z_{i} \dot{z}_{i}+\tilde{\theta}_{f_{i}}^{T} \Gamma_{f_{i}}^{-1} \dot{\tilde{\theta}}_{f_{i}}+\tilde{\theta}_{g_{i}}^{T} \Gamma_{g_{i}}^{-1} \dot{\tilde{\theta}}_{g_{i}}\right)
$$

Using the fact that $\dot{\tilde{\theta}}_{f_{i}}(t)=\dot{\hat{\theta}}_{f_{i}}(t), \dot{\tilde{\theta}}_{g_{i}}(t)=\dot{\hat{\theta}}_{g_{i}}(t)$, and the $z$-coordinate system dynamics defined in (5.30) - (5.33) the time derivative of $V$ becomes

$$
\begin{aligned}
\dot{V}= & z_{1}\left(f_{o_{1}}(x)-\dot{y}_{d}(t)-\dot{\alpha}_{1}\right)+z_{2}\left(\bar{f}_{2}\left(x, \theta_{f_{2}}\right)+\bar{g}_{2}\left(x, \theta_{g_{2}}\right) u-\ddot{y}_{d}(t)-\dot{\alpha}_{2}\right)+ \\
& +z_{3}\left(\bar{f}_{3}\left(x, \theta_{f_{3}}\right)+\bar{g}_{3}\left(x, \theta_{g_{3}}\right) u-y_{d}^{(3)}(t)-\dot{\alpha}_{3}\right)+ \\
& +z_{4}\left(f_{o_{4}}(x)-y_{d}^{(4)}(t)-\dot{\alpha}_{4}\right)+\sum_{i=1}^{n}\left(\tilde{\theta}_{f_{i}}^{T} \Gamma_{f_{i}}^{-1} \dot{\hat{\theta}}_{f_{i}}+\tilde{\theta}_{g_{i}}^{T} \Gamma_{g_{i}}^{-1} \dot{\hat{\theta}}_{g_{i}}\right) .
\end{aligned}
$$

Grouping the terms with the control input $u$ we obtain

$$
\begin{aligned}
\dot{V}= & z_{1}\left(f_{o_{1}}(x)-\dot{y}_{d}(t)-\dot{\alpha}_{1}\right)+z_{2}\left(\bar{f}_{2}\left(x, \theta_{f_{2}}\right)-\ddot{y}_{d}(t)-\dot{\alpha}_{2}\right)+z_{3}\left(\bar{f}_{3}\left(x, \theta_{f_{3}}\right)-\right. \\
& \left.-y_{d}^{(3)}(t)-\dot{\alpha}_{3}\right)+z_{4}\left(f_{o_{4}}(x)-y_{d}^{(4)}(t)-\dot{\alpha}_{4}\right)+\left(z_{2} \bar{g}_{2}\left(x, \theta_{g_{2}}\right)+\right. \\
& \left.+z_{3} \bar{g}_{3}\left(x, \theta_{g_{3}}\right)\right) u+\sum_{i=1}^{n}\left(\tilde{\theta}_{f_{i}}^{T} \Gamma_{f_{i}}^{-1} \dot{\hat{\theta}}_{f_{i}}+\tilde{\theta}_{g_{i}}^{T} \Gamma_{g_{i}}^{-1} \dot{\hat{\theta}}_{g_{i}}\right) .
\end{aligned}
$$

Then substitute in the dynamics from (5.34) - (5.37) with

$$
\dot{V}=z_{1}\left(z_{2}+\alpha_{2}-\dot{\alpha}_{1}\right)+z_{2}\left(z_{3}+\alpha_{3}-x_{3}+\bar{f}_{2}\left(x, \theta_{f_{2}}\right)+\bar{g}_{2}\left(x, \theta_{g_{2}}\right) u-\dot{\alpha}_{2}\right)+
$$




$$
\begin{aligned}
& +z_{3}\left(z_{4}+\alpha_{4}-x_{4}+\bar{f}_{3}\left(x, \theta_{f_{3}}\right)+\bar{g}_{3}\left(x, \theta_{g_{3}}\right) u-\dot{\alpha}_{3}\right)+z_{4}\left(z_{3}-z_{2}+\alpha_{3}-\alpha_{2}-\right. \\
& \left.-\dot{\alpha}_{4}+\ddot{y}_{d}(t)-\dot{y}_{d}(t)-y_{d}^{(4)}(t)\right)+\left(z_{2} \bar{g}_{2}\left(x, \theta_{g_{2}}\right)+z_{3} \bar{g}_{3}\left(x, \theta_{g_{3}}\right)\right) u+ \\
& +\sum_{i=1}^{n}\left(\tilde{\theta}_{f_{i}}^{T} \Gamma_{f_{i}}^{-1} \dot{\hat{\theta}}_{f_{i}}+\tilde{\theta}_{g_{i}}^{T} \Gamma_{g_{i}}^{-1} \dot{\hat{\theta}}_{g_{i}}\right) .
\end{aligned}
$$

Therefore, the intermediate control function $\alpha_{i}$ is determined from (5.43) to be

$$
\begin{aligned}
\alpha_{i}\left(z_{i}, \hat{\theta}_{f_{i}}, \hat{\theta}_{g_{i}}, \hat{\beta}_{i}, y_{d}^{i-1}\right)= & -z_{i-1}-c_{i} z_{i}-f_{o_{i}}-\hat{f}_{i}+\sum_{k=1}^{i-1} \frac{\partial \alpha_{i-1}}{\partial x_{k}}\left(x_{k}+f_{o_{k}}+\hat{f}_{k}\right)+ \\
& +\sum_{k=1}^{i-1} \frac{\partial \alpha_{i-1}}{\partial y_{d}^{k-1}} y_{d}^{(k)} \sum_{k=1}^{i-1} \frac{\partial \alpha_{i-1}}{\partial \hat{\theta}_{k}} \tau_{k_{i}}- \\
& -\sum_{k=1}^{i-1}\left[\frac{\partial \alpha_{i-1}}{\partial x_{k}} \frac{\partial \hat{f}_{k}}{\partial \theta_{k}} \Gamma_{k} \sum_{l=k}^{i-2} \frac{\partial \alpha_{l}^{T}}{\partial \theta_{k}} z_{l+1}\right]
\end{aligned}
$$

where $\tau_{k i}$ are the adaptation functions to be defined in the next subsection.

Adaptive Controller Design. Observing the time derivative of the $V$ function and substituting in the spline based adaptive approximation functions from (5.19) (5.20) for the unknown parts of the dynamics we define the control input law for $u(t)$ to ensure the desired stability properties and tracking objectives

$$
u=\frac{1}{z_{2} \hat{g}_{2}\left(x, \hat{\theta}_{g_{2}}\right)+z_{3} \hat{g}_{3}\left(x, \hat{\theta}_{g_{3}}\right)} \sum_{i=1}^{n}\left(z_{i}\left(y_{d}^{(i)}(t)-\hat{f}_{i}\left(x, \hat{\theta}_{f_{i}}\right)-\dot{\alpha}_{i}\right)-\lambda_{i} z_{i}^{2}\right) .
$$

Next, from the normalized gradient algorithm in [76] we choose the following adaptive laws

$$
\begin{aligned}
& \dot{\hat{\theta}}_{f_{i}}(t)=\tau_{f i}=-\frac{\Gamma_{\theta_{f_{i}}} \phi_{f_{i}} \epsilon_{f_{i}}}{m_{f_{i}}^{2}(t)}, \\
& \dot{\hat{\theta}}_{g_{i}}(t)=\tau_{g i}=-\frac{\Gamma_{\theta_{g_{i}}} \phi_{g_{i}} \epsilon_{g_{i}}}{m_{g_{i}}^{2}(t)},
\end{aligned}
$$

where $\epsilon_{f_{i}}=\hat{\theta}_{f_{i}}^{T} \phi_{f_{i}}, \epsilon_{g_{i}}=\hat{\theta}_{g_{i}}^{T} \phi_{g_{i}}$ and

$$
m_{f_{i}}=\sqrt{1+\kappa_{f_{i}} \phi_{f_{i}}^{T} \phi_{f_{i}}}
$$




$$
m_{g_{i}}=\sqrt{1+\kappa_{g_{i}} \phi_{g_{i}}^{T} \phi_{g_{i}}}
$$

with the $i^{\text {th }}$ constant variables $\kappa_{f_{i}}$, and $\kappa_{g_{i}}$.

Lemma 1 : The closed-loop comprised of the system described by (5.1), with the approximation-based adaptive controller defined by (5.45) and adaptive laws (5.46), (5.47), guarantees the following properties:

1. $z_{i}, x_{i}, \hat{\theta}_{f_{i}}, \hat{\theta}_{g_{i}} \in L_{\infty} \quad i=1,2,3,4$

2. $z \in L^{2}$.

3. $z_{i}(t) \rightarrow 0$ as $t \rightarrow \infty$.

Proof: Recall the positive definite function defined in (5.39) and it's time derivative (5.42) along the signals with the applied control input (5.45), is

$$
\dot{V}=\sum_{i=1}^{n}\left(-\lambda_{i} z_{i}^{2}+\tilde{\theta}_{f_{i}}^{T} \Gamma_{f_{i}}^{-1} \dot{\hat{\theta}}_{f_{i}}+\tilde{\theta}_{g_{i}}^{T} \Gamma_{g_{i}}^{-1} \dot{\hat{\theta}}_{g_{i}}\right) .
$$

Substituting in the parameter adaptation from (5.46), and (5.47), yields the derivative as

$$
\dot{V}=-\sum_{i=1}^{n}\left(\lambda_{i} z_{i}^{2}+\frac{\epsilon_{f_{i}}^{2}}{m_{f_{i}}^{2}}+\frac{\epsilon_{g_{i}}^{2}}{m_{g_{i}}^{2}}\right) .
$$

This corresponds to an adaptive control scheme that ensures desired closed-loop stability and tracking properties which, like those with other approximation based designs, are in a local and average sense due to the approximation errors $\eta_{f_{i}}, \eta_{g_{i}}$, that is, for approximation errors with some non-zero bounds and chosen initial conditions within the specified regions, all closed-loop system signals remain bounded and the $i^{\text {th }} z$-coordinate $z_{i}(t)$ is bounded by the approximation errors in a mean square sense.

Given the fact that each $\eta_{f_{i}}, \eta_{g_{i}}$ is small (in some norm sense) and bounded on a compact region, we have, from (5.51), the boundedness of $z_{i}(t)$, and $\frac{\epsilon_{f_{i}}}{m_{f_{i}}}, \frac{\epsilon_{g_{i}}}{m_{g_{i}}}$. From our aforementioned assumptions, that of $y_{d}(t)$ and, in turn, from $z_{i}(t)=x_{i}(t)-$ $y_{d}^{i-1}(t)-\alpha_{i}$, that of $x_{i}(t)$, from (5.45), that of $u(t)$. Thus all closed-loop signals are bounded. Finally, from (5.46), (5.47), and (5.51), $z_{i}(t)$, each $i^{\text {th }} \dot{\hat{\theta}}_{f_{i}}(t)$, and $\dot{\hat{\theta}}_{g_{i}}(t)$ 
are all bounded by $\eta_{f_{i}}, \eta_{g_{i}}$, respectively, in a mean square sense (e.g. $\int_{t_{1}}^{t_{2}} z_{i}^{2}(t) d t \leq$ $\gamma_{0}+k_{0} \sum_{i=1}^{m} \int_{t_{1}}^{t_{2}} \eta_{i}^{2}(t) d t$ for some constants $\left.\gamma_{0}, k_{0}>0\right)$.

Next, we modify our adaptive control design parameters (5.46), (5.47), to handle the parameter uncertainties outside the region of operation. To do this we will employ a parameter projection technique to ensure that the parameter estimates remain in the bounded region of operation. That is the parameter adaptation laws are defined by projection operators as

$$
\begin{aligned}
& \dot{\hat{\theta}}_{f_{i}}(t)=P_{f_{i}}\left(\tau_{f i}\right)=-P_{f_{i}}\left(\frac{\Gamma_{\theta_{f_{i}}} \phi_{f_{i}} \epsilon_{f_{i}}}{m_{f_{i}}^{2}(t)}\right), \\
& \dot{\hat{\theta}}_{g_{i}}(t)=P_{g_{i}}\left(\tau_{g i}\right)=-P_{g_{i}}\left(\frac{\Gamma_{\theta_{g_{i}}} \phi_{g_{i}} \epsilon_{g_{i}}}{m_{g_{i}}^{2}(t)}\right),
\end{aligned}
$$

The parameter projection operators $P_{f_{i}}, P_{g_{i}}$ for $i=1,2,3,4$ are used to maintain the boundedness of the elements for each $\hat{\theta}_{f_{i}}$ and $\hat{\theta}_{g_{i}}$. Applying the fact that for spline functions the basis elements $B_{f_{i}}$ and $B_{g_{i}}$ defined previously in equations (5.17) and (5.18) form a partition of unity on $D$. From this it is straightforward to define the upper and lower bounds on each element of $\hat{\theta}_{f_{i}}$ and $\hat{\theta}_{g_{i}}$ such that the inequalities hold $\underline{\theta_{f_{j i}}} \leq \hat{\theta}_{f_{j i}} \leq \bar{\theta}_{f_{j i}}, \underline{\theta_{g_{j i}}} \leq \hat{\theta}_{g_{j i}} \leq \bar{\theta}_{g_{j i}}$ which ensure the boundedness of $\hat{\theta}_{f_{i}}$ and $\hat{\theta}_{g_{i}}$, respectively [19]. Therefore, each $P_{f_{i}}, P_{g_{i}}$ projection operator performs component wise according to the definitions

$$
\begin{aligned}
& P_{f_{j i}}\left(\tau_{f i}\right)= \begin{cases}\tau_{f i} & \theta_{f_{j i}} \leq \hat{\theta}_{f_{j i}} \leq \bar{\theta}_{f_{j i}} \\
0 & \text { otherwise }\end{cases} \\
& P_{g_{j i}}\left(\tau_{g i}\right)= \begin{cases}\tau_{g i} & \underline{\theta_{g_{j i}}} \leq \hat{\theta}_{g_{j i}} \leq \bar{\theta}_{g_{j i}} \\
0 & \text { otherwise }\end{cases}
\end{aligned}
$$

\subsubsection{Simulations}

This section presents the simulation results for the spline function approximation based adaptive control designs presented in the first part of Chapter 5. To begin our simulation results, we determine system performance by measuring the tracking error 
$e(t)=y(t)-y_{d}(t)$ for a sinusoidal reference input chosen as $y_{d}(t)=\frac{2 \pi}{180} \sin t$. This corresponds to an amplitude of $2^{\circ}$ and a frequency of $\omega=2 \pi f=1(\mathrm{rad} / \mathrm{sec})$. The physical parameters used in the simulations are $m=480 \mathrm{~kg}, I=400 \mathrm{~kg} \cdot \mathrm{m}^{2}, m_{f}=$ $50 \mathrm{~kg}, I_{f}=10 \mathrm{~kg} \cdot \mathrm{m}^{2}, b=1.5 \mathrm{~m}, d=1.25 \mathrm{~m}, a=0.21 \mathrm{~m}, F=2300 \mathrm{~N}$, and $\epsilon=3.7 \mathrm{~kg}$. $\mathrm{m}^{2} / \mathrm{s}$, as given in [53]. The spacecraft model with fuel slosh was chosen to have initial conditions of the system as $\theta_{0}=2^{\circ}, \dot{\theta}_{0}=0.037(\mathrm{deg} / \mathrm{sec}), \psi_{0}=5^{\circ}$, and $\dot{\theta}_{f 0}=0$. The following simulation shows the tracking error $y(t)-y_{d}(t)$ for the spline function based adaptive control design presented in Section 5.1, applied to the SISO spacecraft model with fuel slosh.

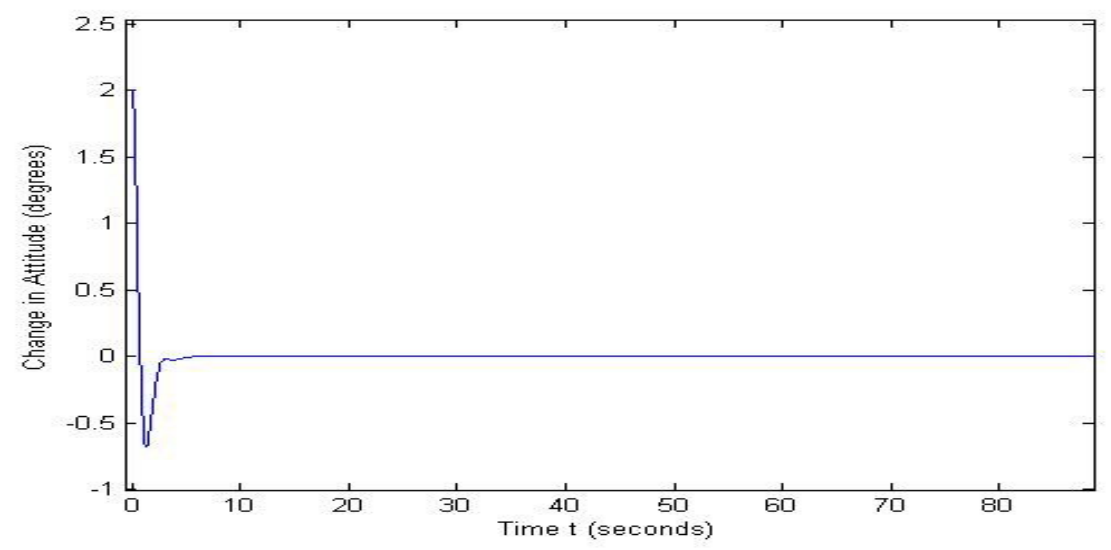

Figure 5.1: Tracking error $y(t)-y_{d}(t)$ for $y_{d}(t)=\frac{2 \pi}{180} \sin t$ : SISO spacecraft model with fuel slosh.

Next, we simulate the case for $y_{d}(t)=\frac{2 \pi}{180} \sin t+\frac{4 \pi}{180} \cos t$, this is shown in Figure 5.1 and verifies that indeed the tracking error goes to zero.

Although the transient error increases the steady-state error goes to zero. In order to shed some light on how practical this controller design is, we present the control input $u(t)$ displayed in Figure 5.3, that is applied to achieve the tracking error that is presented in Figure 5.2. To apply this controller to a real spacecraft we would have to consider the response time for modern control inputs. In addition, we would study the frequency of which these control inputs can operate and design the adaptive 


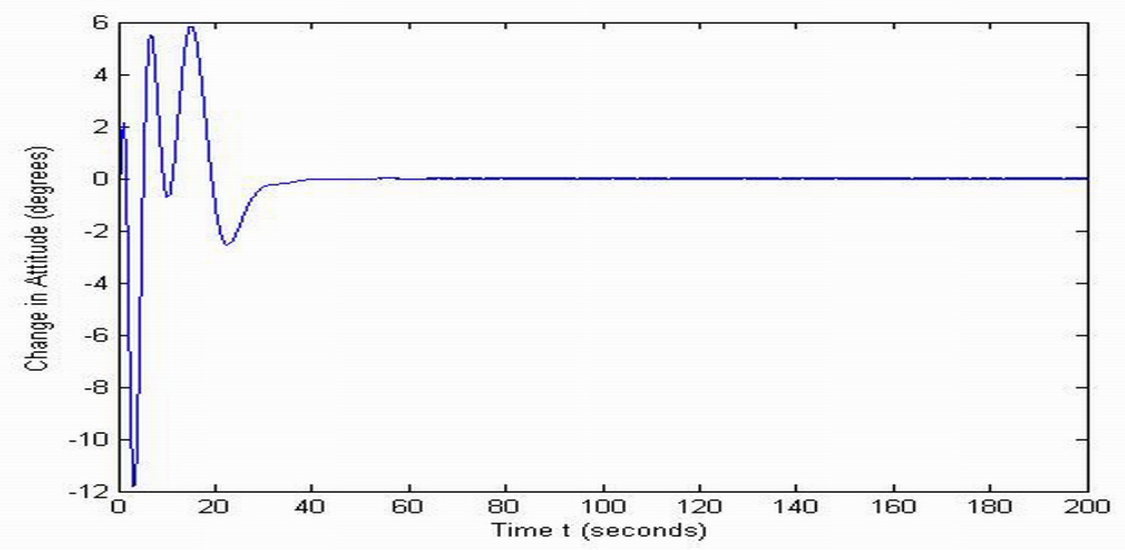

Figure 5.2: Tracking error $y(t)-y_{d}(t)$ for $y_{d}(t)=\frac{2 \pi}{180} \sin t+\frac{4 \pi}{180} \cos t$ : SISO spacecraft model with fuel slosh.

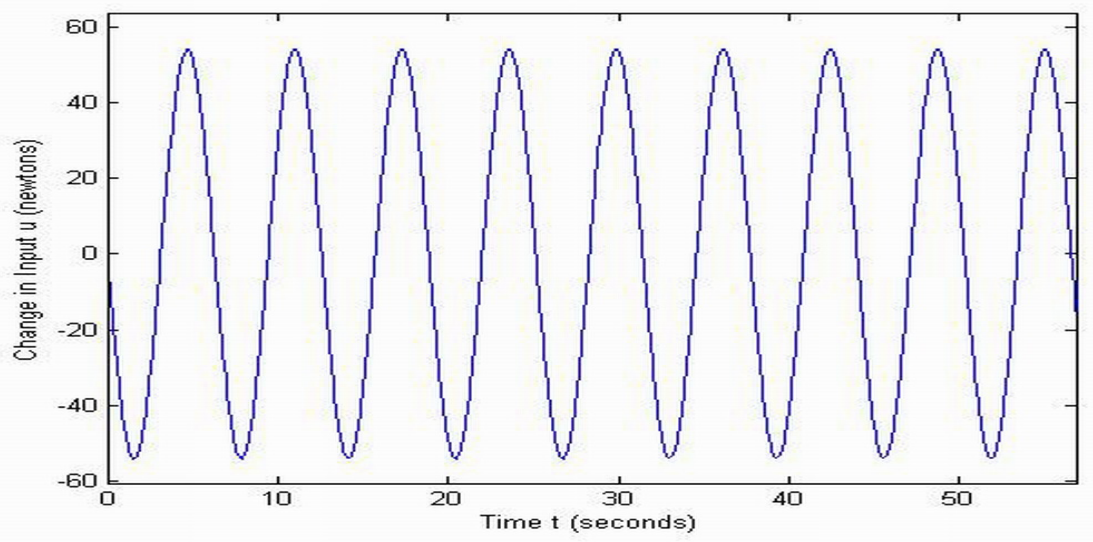

Figure 5.3: Input $u(t)$ for the tracking error $y(t)-y_{d}(t)$ shown in Figure 5.2. 
controller to maintain the control input $u(t)$ to meet the frequency requirements. In addition to the tracking error we simulated the responses for the output $\theta(t)$ shown in Figure 5.4 the spacecraft attitude, $\psi(t)$ the angle of the fuel slosh shown in Figure 5.5. The purpose of simulating these states is to show that they are indeed bounded for the chosen time set.

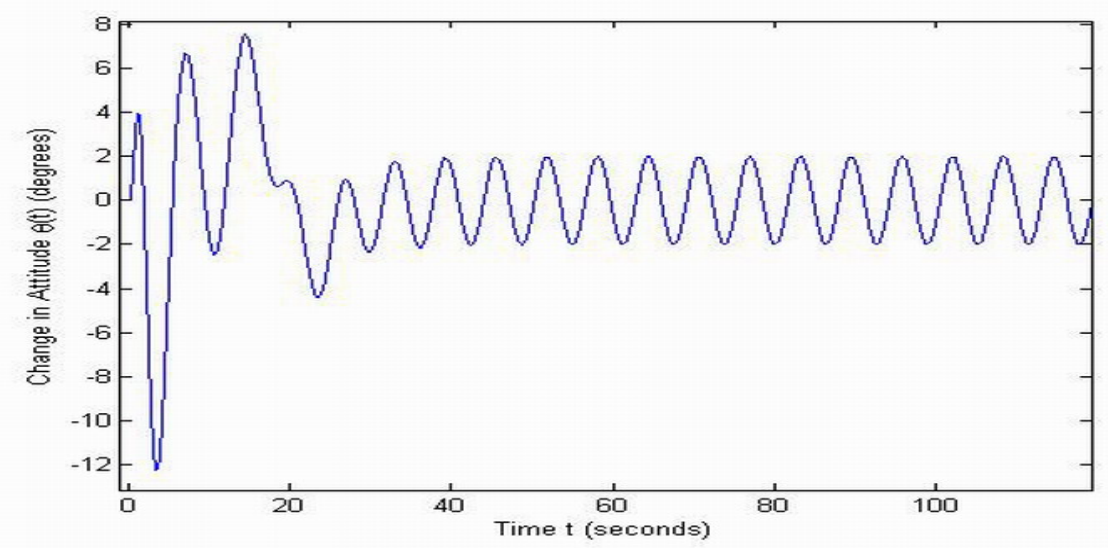

Figure 5.4: Output response for $y(t)=\theta(t)$ and $y_{d}(t)=\frac{2 \pi}{180} \sin t$ : SISO spacecraft model with fuel slosh.

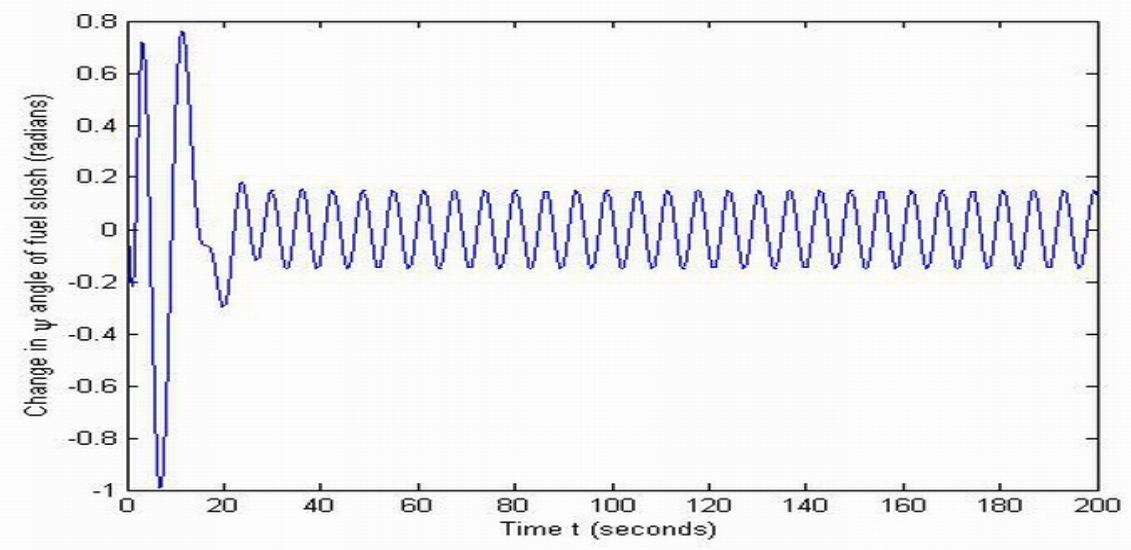

Figure 5.5: Slosh angle response $\psi(t)$, for $y_{d}(t)=\frac{2 \pi}{180} \sin t$ : SISO spacecraft model with fuel slosh. 


\subsection{Approximation and Control of MIMO Model}

Modifying the MIMO spacecraft model presented in Section 3.2, to include an additional input that allows for more precise attitude tracking. The new model now has three inputs for control purposes a transverse force $F_{T}$, a pitching moment $M$ and $\delta$ denotes the gimbal deflection angle with the longitudinal thrust $F_{L}$ as shown in Figure 5.6.

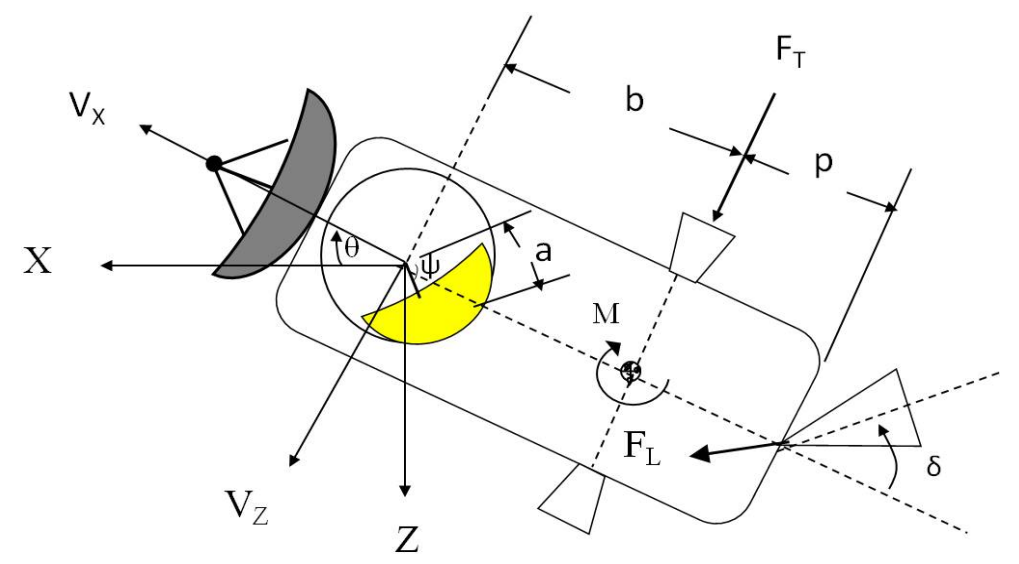

Figure 5.6: Modified MIMO Spacecraft with fuel slosh dynamics [53].

The modified design variables are defined as

$$
\begin{gathered}
R=\frac{1}{2} \epsilon \dot{\psi}^{2}, v=\left(\begin{array}{c}
v_{x} \\
0 \\
v_{z}
\end{array}\right), \omega=\left(\begin{array}{c}
0 \\
\dot{\theta} \\
0
\end{array}\right), \\
\tau_{t}=\left(\begin{array}{c}
F_{L} \cos \delta \\
0 \\
F_{T}+F_{L} \sin \delta
\end{array}\right), \tau_{r}=\left(\begin{array}{c}
0 \\
M+F_{T} b+F_{L} l \sin \delta \\
0
\end{array}\right) .
\end{gathered}
$$

For simplicity we introduce the length $l=b+p$. After solving the Lagrange-Euler equations, we have the following equations of motion for the spacecraft with fuel sloshing dynamics included:

$$
F_{L} \cos \delta=m_{f} a(\ddot{\theta}+\ddot{\psi}) \sin \psi+m_{f} a(\dot{\theta}+\dot{\psi})^{2} \cos \psi+
$$




$$
\begin{gathered}
+m b \dot{\theta}^{2}+\left(m+m_{f}\right)\left(\dot{v}_{x}+\dot{\theta} v_{z}\right) \\
F_{T}+F_{L} \sin \delta=\left(m+m_{f}\right)\left(\dot{v}_{z}-\dot{\theta} v_{x}\right)+m b \ddot{\theta}+m_{f} a(\ddot{\theta}+ \\
+\ddot{\psi}) \cos \psi-m_{f} a(\dot{\theta}+\dot{\psi})^{2} \sin \psi \\
0=\left(I_{f}+m_{f} a^{2}\right)(\ddot{\theta}+\ddot{\psi})+m_{f} a\left(\dot{v}_{x}+\dot{\theta} v_{z}\right) \sin \psi+ \\
+m_{f} a\left(\dot{v}_{z}-\dot{\theta} v_{x}\right) \cos \psi+\epsilon \dot{\psi}, \\
M+F_{T} b+F_{L} l \sin \delta=\left(I+m b^{2}\right) \ddot{\theta}+m b\left(\dot{v}_{z}-\dot{\theta} v_{x}\right) .
\end{gathered}
$$

Since the focus is on the effect of fuel slosh during maneuvering, the angle $\theta$ representing the attitude is important to the simulation. For the longitudinal thruster we assume small gimbal deflection of $\delta$ so that $\cos \delta \approx 1$ and $\sin \delta \approx \delta$. This allows $F_{L}$ to primarily control the orbit position and the distance at which the spacecraft travels in the x-direction. The transverse thrusters $F_{T}$ control the movement on the z-axis, while the pitching moment $M$ controls the rotation of the craft in a fixed plane. The nonlinear state-variable form is complex and can be derived by first declaring the states of the system, which in our case will be the position and velocity variables for our spacecraft. To place the system in state-variable form we introduce the following state variables:

$$
x_{1}=v_{x}, x_{2}=v_{z}, x_{3}=\theta, x_{4}=\dot{\theta}, x_{5}=\psi, x_{6}=\dot{\psi}
$$

With the state variables defined, we write our nonlinear equations in state-variable form by plugging in each corresponding state for the chosen variables:

$$
\begin{aligned}
\dot{x} & =f(x, u), \\
y & =h(x),
\end{aligned}
$$


where

$$
u=\left[\begin{array}{c}
\delta \\
F_{T} \\
M
\end{array}\right], \quad y=\left[\begin{array}{c}
v_{z} \\
\theta \\
\psi
\end{array}\right] .
$$

The system is governed by the set of equations (5.54)-(5.57), in compact form:

$$
\left[\begin{array}{c}
\dot{v}_{x} \\
\dot{v}_{z} \\
\ddot{\theta} \\
\ddot{\psi}
\end{array}\right]=N^{-1} G_{x}+N^{-1} G_{u} u
$$

where

$$
\begin{gathered}
G_{x}=\left[\begin{array}{c}
F_{L}-m b \dot{\theta}^{2}-\left(m+m_{f}\right) \dot{\theta} v_{z}-m_{f} a(\dot{\theta}+\dot{\psi})^{2} \cos \psi \\
\left(m+m_{f}\right) \dot{\theta} v_{x}+m_{f} a(\dot{\theta}+\dot{\psi})^{2} \sin \psi \\
m b \dot{\theta} v_{x} \\
-\epsilon \dot{\psi}-m_{f} a \dot{\theta} v_{z} \sin \psi+m_{f} a \dot{\theta} v_{x} \cos \psi
\end{array}\right], \\
G_{u}=\left[\begin{array}{ccc}
0 & 0 & 0 \\
F_{L} & 1 & 0 \\
F_{L} l & b & 1 \\
0 & 0 & 0
\end{array}\right], \quad N= \\
m_{+} \\
0
\end{gathered}
$$

After performing some rigorous math (see [65]) we obtain the nonlinear system in affine control form

$$
\begin{aligned}
\dot{x} & =f(x)+g(x) u, \\
y & =h(x),
\end{aligned}
$$


where

$$
f(x)=\left[\begin{array}{c}
f_{1}(x) \\
f_{2}(x) \\
x_{4} \\
f_{4}(x) \\
x_{6} \\
f_{6}(x)
\end{array}\right], g(x)=\left[\begin{array}{ccc}
g_{11}(x) & g_{12}(x) & g_{13}(x) \\
g_{21}(x) & g_{22}(x) & g_{23}(x) \\
0 & 0 & 0 \\
g_{41}(x) & g_{42}(x) & g_{43}(x) \\
0 & 0 & 0 \\
g_{61}(x) & g_{62}(x) & g_{63}(x)
\end{array}\right],
$$

and $h(x)=\left[0, x_{2}, x_{3}, 0, x_{5}, 0\right]^{T}$ as the system output.

Reduced Order System Model. This full order system is quite complex and highly demanding, therefore in this dissertation we will study a realistically reduced order system with the logical assumption that the pitch and slosh dynamics have negligible influence on the axial acceleration [11]. First we rewrite the system equation $(5.54)$ as

$$
\begin{aligned}
\dot{v}_{x}+\dot{\theta} v_{z}= & \frac{1}{m+m_{f}}\left[F_{L}-m_{f} a(\ddot{\theta}+\ddot{\psi}) \sin \psi-m b \dot{\theta}^{2}-\right. \\
& \left.-m_{f} a(\dot{\theta}+\dot{\psi})^{2} \cos \psi\right] .
\end{aligned}
$$

The stated assumption allows us to simplify this to

$$
\dot{v}_{x}+\dot{\theta} v_{z}=\frac{F_{L}}{m+m_{f}},
$$

and plugging this into equation (5.56), we obtain the following reduced order model

$$
\begin{gathered}
F_{T}+F_{L} \delta=\left(m+m_{f}\right)\left(\dot{v}_{z}-\dot{\theta} v_{x}\right)+m_{f} a(\ddot{\theta}+\ddot{\psi}) \cos \psi+ \\
+m b \ddot{\theta}-m_{f} a(\dot{\theta}+\dot{\psi})^{2} \sin \psi, \\
0=\left(I_{f}+m_{f} a^{2}\right)(\ddot{\theta}+\ddot{\psi})+m_{f} a\left(\frac{F_{L}}{m+m_{f}}\right) \sin \psi+ \\
+m_{f} a\left(\dot{v}_{z}-\dot{\theta} v_{x}\right) \cos \psi+\epsilon \dot{\psi}, \\
M+F_{T} b+F_{L} l \delta=\left(I+m b^{2}\right) \ddot{\theta}+m b\left(\dot{v}_{z}-\dot{\theta} v_{x}\right) .
\end{gathered}
$$


Now $v_{x}(t)$ is considered as an exogenous input. To place the reduced order system in state-variable form we define:

$$
x_{1}=v_{z}, \quad x_{2}=\theta, \quad x_{3}=\dot{\theta}, \quad x_{4}=\psi, \quad x_{5}=\dot{\psi}
$$

and write out the nonlinear equations (5.63)-(5.65) in state-variable form:

$$
\begin{aligned}
\dot{x} & =f(x, u), \\
y & =h(x),
\end{aligned}
$$

where

$$
u=\left[\begin{array}{c}
\delta \\
F_{T} \\
M
\end{array}\right], \quad y=\left[\begin{array}{c}
v_{z} \\
\theta \\
\psi
\end{array}\right] .
$$

Which can be represented in compact form:

$$
\left[\begin{array}{c}
\dot{v}_{z} \\
\ddot{\theta} \\
\ddot{\psi}
\end{array}\right]=N^{-1} G_{x}+N^{-1} G_{u} u
$$

where

$$
\begin{gathered}
G_{x}=\left[\begin{array}{c}
\left(m+m_{f}\right) \dot{\theta} v_{x}(t)+m_{f} a(\dot{\theta}+\dot{\psi})^{2} \sin \psi \\
m b \dot{\theta} v_{x}(t) \\
-\epsilon \dot{\psi}-\frac{m_{f} a F_{L}}{m+m_{f}} \sin \psi+m_{f} a \dot{\theta} v_{x}(t) \cos \psi
\end{array}\right], \\
G_{u}=\left[\begin{array}{ccc}
F_{L} & 1 & 0 \\
F_{L} l & b & 1 \\
0 & 0 & 0
\end{array}\right], \quad N=\left[\begin{array}{ccc}
m+m_{f} & m_{f} a \cos \psi+m b & m_{f} a \cos \psi \\
m b & I+m b^{2} & 0 \\
m_{f} a \cos \psi & I_{f}+m_{f} a^{2} & I_{f}+m_{f} a^{2}
\end{array}\right] .
\end{gathered}
$$

Recall the formula to solve for the inverse of a square matrix:

$$
N^{-1}=\frac{1}{\operatorname{det}(N)} \operatorname{adj}(N)
$$


To find the $\operatorname{adj}(N)$ we first find the cofactor of each element of $N$ and take the transpose of the cofactor matrix of $N$, this results

$$
\operatorname{adj}(N)=\left[\begin{array}{lll}
N_{11} & N_{21} & N_{31} \\
N_{12} & N_{22} & N_{32} \\
N_{13} & N_{23} & N_{33}
\end{array}\right] .
$$

Through some rigorous math we obtain

$$
\begin{array}{lc}
N_{11}= & \left(I+m b^{2}\right)\left(I_{f}+m_{f} a^{2}\right), \\
N_{12}= & -m b\left(I_{f}+m_{f} a^{2}\right), \\
N_{13}= & m b\left(I_{f}+m_{f} a^{2}\right)-\left(I+m b^{2}\right) m_{f} a \cos x_{4}, \\
N_{21}= & -m b\left(I_{f}+m_{f} a^{2}\right), \\
N_{22}= & \left(m+m_{f}\right)\left(I_{f}+m_{f} a^{2}\right)-m_{f}^{2} a^{2} \cos ^{2} x_{4}, \\
N_{23}= & \left(m b+m_{f} a \cos x_{4}\right) m_{f} a \cos x_{4}-\left(I_{f}+m_{f} a^{2}\right)\left(m+m_{f}\right), \\
N_{31}= & -\left(I+m b^{2}\right) m_{f} a \cos x_{4}, \\
N_{32}= & m b m_{f} a \cos x_{4}, \\
N_{33}= & \left(I+m b^{2}\right)\left(m+m_{f}\right)-m b\left(m b+m_{f} a \cos x_{4}\right) .
\end{array}
$$

This assists us in solving for the determinant of $N$

$$
D_{N}=\operatorname{det}(N)=m_{f} a \cos x_{4} N_{13}+\left(I_{f}+m_{f} a^{2}\right) N_{33},
$$

for simplicity we introduce

$$
D n_{i, j}=\frac{N_{i, j}}{D_{N}}, i, j=1,2,3
$$

and rewrite $G_{x}, G_{u}$ as

$$
G_{x}=\left[\begin{array}{c}
G_{1 x}(x) \\
G_{2 x}(x) \\
G_{3 x}(x)
\end{array}\right], G_{u}=\left[\begin{array}{ccc}
G_{1 u 1} & G_{1 u 2} & 0 \\
G_{2 u 1} & G_{2 u 2} & G_{2 u 3} \\
0 & 0 & 0
\end{array}\right],
$$

with

$$
\begin{aligned}
& G_{1 x}(x)=\left(m+m_{f}\right) x_{3} v_{x}(t)+m_{f} a\left(x_{3}+x_{5}\right)^{2} \sin x_{4}, \\
& G_{2 x}(x)=m b x_{3} v_{x}(t)
\end{aligned}
$$




$$
\begin{aligned}
G_{3 x}(x) & =-\epsilon x_{5}-\frac{m_{f} a F_{L}}{m+m_{f}} \sin x_{4}+m_{f} a x_{3} v_{x}(t) \cos x_{4} \\
G_{1 u 1} & =F_{L}, \quad G_{1 u 2}=1, \quad G_{2 u 1}=F_{L} l \\
G_{2 u 2} & =b, \quad G_{2 u 3}=1 .
\end{aligned}
$$

This enables us to write out the nonlinear system in affine control form as

$$
\begin{aligned}
\dot{x} & =f(x)+g(x) u \\
y & =h(x)
\end{aligned}
$$

where

$$
f(x)=\left[\begin{array}{c}
f_{1}(x) \\
x_{3} \\
f_{2}(x) \\
x_{5} \\
f_{3}(x)
\end{array}\right], g(x)=\left[\begin{array}{ccc}
g_{11}(x) & g_{12}(x) & g_{13}(x) \\
0 & 0 & 0 \\
g_{21}(x) & g_{22}(x) & g_{23}(x) \\
0 & 0 & 0 \\
g_{31}(x) & g_{32}(x) & g_{33}(x)
\end{array}\right]
$$

with

$$
\begin{aligned}
f_{1}(x) & =G_{1 x}(x) D n_{1,1}+G_{2 x}(x) D n_{2,1}+G_{3 x}(x) D n_{3,1} \\
f_{2}(x) & =G_{1 x}(x) D n_{1,2}+G_{2 x}(x) D n_{2,2}+G_{3 x}(x) D n_{3,2}, \\
f_{3}(x) & =G_{1 x}(x) D n_{1,3}+G_{2 x}(x) D n_{2,3}+G_{3 x}(x) D n_{3,3} \\
g_{11}(x) & =G_{1 u 1} D n_{1,1}+G_{2 u 1} D n_{2,1} \\
g_{12}(x) & =G_{1 u 2} D n_{1,1}+G_{2 u 2} D n_{2,1} \\
g_{13}(x) & =G_{2 u 3} D n_{2,1} \\
g_{21}(x) & =G_{1 u 1} D n_{1,2}+G_{2 u 1} D n_{2,2} \\
g_{22}(x) & =G_{1 u 2} D n_{1,2}+G_{2 u 2} D n_{2,2} \\
g_{23}(x) & =G_{2 u 3} D n_{2,2} \\
g_{31}(x) & =G_{1 u 1} D n_{1,3}+G_{2 u 1} D n_{2,3} \\
g_{32}(x) & =G_{1 u 2} D n_{1,3}+G_{2 u 2} D n_{2,3} \\
g_{33}(x) & =G_{2 u 3} D n_{2,3}
\end{aligned}
$$

and $h(x)=\left[x_{1}, x_{2}, 0, x_{4}, 0\right]^{T}$ as the system output. 


\subsubsection{Splines for Approximation and Control}

We are now ready to develop our spline function based adaptive control design for the MIMO reduced order spacecraft model with fuel slosh. The first step here is to rewrite the system representation with each uncertain function from equation (5.69) modeled using spline functions. That is, the uncertain nonlinear system functions will be approximated by multivariable B-splines defined as

$$
f_{i}(x) \triangleq f_{i}^{*}\left(x, \theta_{f_{i}}^{*}\right) \approx \theta_{f_{i}}^{* T} B_{f_{i}}(x)+\eta_{f_{i}}(x)
$$

and for each control input

$$
\begin{aligned}
& g_{1 i}(x) \triangleq g_{1 i}^{*}\left(x, \theta_{g_{1 i}}^{*}\right) \approx \theta_{g_{1 i}}^{* T} B_{g_{1 i}}(x)+\eta_{g_{1 i}}(x), \\
& g_{2 i}(x) \triangleq g_{2 i}^{*}\left(x, \theta_{g_{2 i}}^{*}\right) \approx \theta_{g_{2 i}}^{* T} B_{g_{2 i}}(x)+\eta_{g_{2 i}}(x) . \\
& g_{3 i}(x) \triangleq g_{3 i}^{*}\left(x, \theta_{g_{3 i}}^{*}\right) \approx \theta_{g_{3 i}}^{* T} B_{g_{3 i}}(x)+\eta_{g_{3 i}}(x) .
\end{aligned}
$$

Where the expressions are,

$$
\theta_{f_{i}}^{* T} B_{f_{i}}(x)=\underbrace{\sum_{j_{1}=1}^{M_{1}} \cdots \sum_{j_{3}=1}^{M_{3}}}_{3} \theta_{j_{1} \cdots j_{3}}^{*} b_{j_{1}}\left(x_{1}\right) \cdots b_{j_{3}}\left(x_{3}\right),
$$

and

$$
\begin{aligned}
& \theta_{g_{1 i}}^{* T} B_{g_{1 i}}(x)=\underbrace{\sum_{j_{1}=1}^{M_{1}} \cdots \sum_{j_{3}=1}^{M_{3}} \theta_{j_{1} \cdots j_{3}}^{*} b_{j_{1}}\left(x_{1}\right) \cdots b_{j_{3}}\left(x_{3}\right),}_{3} \\
& \theta_{g_{2 i}}^{* T} B_{g_{2 i}}(x)=\underbrace{\sum_{j_{1}=1}^{M_{1}} \cdots \sum_{j_{3}=1}^{M_{3}} \theta_{j_{1} \cdots j_{3}}^{*} b_{j_{1}}\left(x_{1}\right) \cdots b_{j_{3}}\left(x_{3}\right),}_{3} \\
& \theta_{g_{3 i}}^{* T} B_{g_{3 i}}(x)=\underbrace{\sum_{j_{1}=1}^{M_{1}} \cdots \sum_{j_{3}=1}^{M_{3}}}_{3} \theta_{j_{1} \cdots j_{3}}^{*} b_{j_{1}}\left(x_{1}\right) \cdots b_{j_{3}}\left(x_{3}\right),
\end{aligned}
$$

Here the index $i=1,2,3$, are the number of uncertain system functions defined in (5.69), in order to reduce the modeling error effects on the tracking objectives they will be approximated by the smooth functions $\hat{f}_{i}\left(x, \hat{\theta}_{f_{i}}\right), \hat{g}_{1 i}\left(x, \hat{\theta}_{g_{1 i}}\right), \hat{g}_{2 i}\left(x, \hat{\theta}_{g_{2 i}}\right)$ and $\hat{g}_{3 i}\left(x, \hat{\theta}_{g_{3 i}}\right)$, respectively. 
The vectors $\hat{\theta}_{f_{i}} \in \Re^{q_{f}}, \hat{\theta}_{g_{1 i}} \in \Re^{q_{g}}, \hat{\theta}_{g_{2 i}} \in \Re^{q_{g}}$ and $\hat{\theta}_{g_{3 i}} \in \Re^{q_{g}}$ represent the adjustable parameters for each approximating function, where $\theta_{f_{i}}^{*}, \theta_{g_{1 i}}^{*}, \theta_{g_{2 i}}^{*}$ and $\theta_{g_{3 i}}^{*}$ are the corresponding unknown "optimal" parameter (weight) vectors. $\eta_{f_{i}}, \eta_{g_{1 i}}, \eta_{g_{2 i}}$ and $\eta_{g_{3 i}}$ represent the $i^{t h}$ reconstruction errors and are defined as

$$
\begin{aligned}
\eta_{f_{i}}(x) & =f_{i}(x)-f_{i}^{*}\left(x, \theta_{f_{i}}^{*}\right), \\
\eta_{g_{1 i}}\left(x, u_{1}\right) & =g_{1 i}(x) u_{1}-g_{1 i}^{*}\left(x, \theta_{g_{1 i}}^{*}\right) u_{1}, \\
\eta_{g_{2 i}}\left(x, u_{2}\right) & =g_{2 i}(x) u_{2}-g_{2 i}^{*}\left(x, \theta_{g_{2 i}}^{*}\right) u_{2}, \\
\eta_{g_{3 i}}\left(x, u_{3}\right) & =g_{3 i}(x) u_{3}-g_{3 i}^{*}\left(x, \theta_{g_{3 i}}^{*}\right) u_{3} .
\end{aligned}
$$

For equation 5.78 we define an upper bound on the magnitude of $\eta_{f_{i}}(x)$ that is assumed to be unknown and is given by

$$
\eta_{f f_{i}}^{*}=\sup _{x \in \Omega}\left\|\eta_{f_{i}}(x)\right\|
$$

This case requires an adaptive bounding scheme to update the estimates on the bounds for $\eta_{f_{i}}(x)$ on-line. This estimate is defined as $\hat{\eta}_{f f_{i}}$, with the bounding estimation error defined as

$$
\tilde{\eta}_{f f_{i}}(t)=\hat{\eta}_{f f_{i}}(t)-\eta_{f f_{i}}^{*}
$$

which will we be applied in the stability analysis. However, for the remaining reconstruction errors $(5.79,5.80,5.81)$, we assume that the bounds on the functions which multiply the control inputs are known and are bounded by reasonably determined positive constants $\left\|\eta_{g_{1 i}}\right\| \leq \eta_{g g_{1 i}},\left\|\eta_{g_{2 i}}\right\| \leq \eta_{g g_{2 i}}$ and $\left\|\eta_{g_{3 i}}\right\| \leq \eta_{g g_{3 i}}$, which approach zero in a cubic fashion. $\theta_{j_{1} j_{2} j_{3}}^{*}$ for each $i^{\text {th }}$ approximator is one of $\left(M_{1} \cdot M_{2} \cdot M_{3}\right)$ unknown spline coefficients, and $b_{j_{1}}\left(x_{1}\right), b_{j_{2}}\left(x_{2}\right), b_{j_{3}}\left(x_{3}\right)$, are the three univariable spline basis elements, and the approximations of the uncertain functions for each $i^{\text {th }}$ model is

$$
\begin{gathered}
\hat{f}_{i}\left(x ; \hat{\theta}_{f_{i}}\right)=\hat{\theta}_{f_{i}}^{T} B_{f_{i}}(x), \\
\hat{g}_{1 i}\left(x ; \hat{\theta}_{g_{1 i}}\right)=\hat{\theta}_{g_{1 i}}^{T} B_{g_{1 i}}(x), \\
\hat{g}_{2 i}\left(x ; \hat{\theta}_{g_{2 i}}\right)=\hat{\theta}_{g_{2 i}}^{T} B_{g_{2 i}}(x),
\end{gathered}
$$

and

$$
\hat{g}_{3 i}\left(x ; \hat{\theta}_{g_{3 i}}\right)=\hat{\theta}_{g_{3 i}}^{T} B_{g_{3 i}}(x) .
$$


Hence, we define the $i^{t h}$ parameter estimation errors $\tilde{\theta}_{f_{i}}(t)=\hat{\theta}_{f_{i}}(t)-\theta_{f_{i}}^{*}, \tilde{\theta}_{g_{1 i}}(t)=$ $\hat{\theta}_{g_{1 i}}(t)-\theta_{g_{1 i}}^{*}, \tilde{\theta}_{g_{2 i}}(t)=\hat{\theta}_{g_{2 i}}(t)-\theta_{g_{2 i}}^{*}$ and $\tilde{\theta}_{g_{3 i}}(t)=\hat{\theta}_{g_{3 i}}(t)-\theta_{g_{3 i}}^{*}$. As the $i^{t h}$ estimates $\hat{\theta}_{f_{i}}^{T}$, $\hat{\theta}_{g_{1 i}}^{T}, \hat{\theta}_{g_{2 i}}^{T}$ and $\hat{\theta}_{g_{3 i}}^{T}$ approach the desired parameters $\theta_{f_{i}}^{* T}, \theta_{g_{1 i}}^{* T}, \theta_{g_{2 i}}^{* T}$ and $\theta_{g_{3 i}}^{* T}$, respectively, the spline function approximator structure effectively provides a model for spacecraft with fuel slosh dynamics that can be used for control purposes. Observing the form of these error expressions is critical for the adaptive controller design such as to guarantee closed-loop stability.

Adaptive Control Design. Next, we define the tracking errors for each output, recall from the system model (5.69) the first output $y_{1}(x)=x_{1}=v_{z}$ which is the velocity component in the $z$-plane, the second output $y_{2}(x)=x_{2}=\theta$ the spacecraft attitude and the third output $y_{3}(x)=x_{4}=\psi$ the pendulum fuel slosh angle. Thus, the output tracking errors are $e_{1}=y_{1}-y_{d_{1}}, e_{2}=y_{2}-y_{d_{2}}$ and $e_{3}=y_{3}-y_{d_{3}}$ for each corresponding output, where $y_{d_{1}}, y_{d_{2}}$ and $y_{d_{3}}$ are the desired tracking functions. These errors are applied using a sliding mode vector of each component

$$
s=\left[\begin{array}{c}
s_{1} \\
s_{2} \\
s_{3}
\end{array}\right]=\left[\begin{array}{c}
e_{1}+c_{1} \int e_{1} d \tau \\
e_{2}+c_{2} \int e_{2} d \tau \\
e_{3}+c_{3} \int e_{3} d \tau
\end{array}\right],
$$

where $c_{1}, c_{2}$ and $c_{3}$ are positive design constants. Each of the $s$ components are essentially filtered error signals that induce proportional and integral control action [36, 69]. The adaptive laws are reminiscent to what has been presented in [50] to update the parameter vectors $\hat{\theta}_{f_{i}}, \hat{\theta}_{g_{1 i}}, \hat{\theta}_{g_{2 i}}$ and $\hat{\theta}_{g_{3 i}}$ and are defined by:

$$
\begin{aligned}
& \dot{\hat{\theta}}_{f_{1}}=\gamma_{f_{1}}\left\{B_{f_{1}} s_{1}-\sigma_{1}\left(\hat{\theta}_{f_{1}}-\theta_{f_{1}}^{0}\right)\right\}, \\
& \dot{\hat{\theta}}_{f_{2}}=\gamma_{f_{2}}\left\{B_{f_{2}} s_{2}-\sigma_{2}\left(\hat{\theta}_{f_{2}}-\theta_{f_{2}}^{0}\right)\right\}, \\
& \dot{\hat{\theta}}_{f_{3}}=\gamma_{f_{3}}\left\{B_{f_{3}} s_{3}-\sigma_{3}\left(\hat{\theta}_{f_{3}}-\theta_{f_{3}}^{0}\right)\right\},
\end{aligned}
$$

and

$$
\begin{aligned}
& \dot{\hat{\theta}}_{g_{11}}=\gamma_{g_{11}}\left\{B_{g_{11}} s_{1}-\sigma_{11}\left(\hat{\theta}_{g_{11}}-\theta_{g_{11}}^{0}\right)\right\}, \\
& \dot{\hat{\theta}}_{g_{21}}=\gamma_{g_{21}}\left\{B_{g_{21}} s_{1}-\sigma_{21}\left(\hat{\theta}_{g_{21}}-\theta_{g_{21}}^{0}\right)\right\}, \\
& \dot{\hat{\theta}}_{g_{31}}=\gamma_{g_{31}}\left\{B_{g_{31}} s_{1}-\sigma_{31}\left(\hat{\theta}_{g_{31}}-\theta_{g_{31}}^{0}\right)\right\},
\end{aligned}
$$




$$
\begin{aligned}
& \dot{\hat{\theta}}_{g_{12}}=\gamma_{g_{12}}\left\{B_{g_{12}} s_{2}-\sigma_{12}\left(\hat{\theta}_{g_{12}}-\theta_{g_{12}}^{0}\right)\right\}, \\
& \dot{\hat{\theta}}_{g_{22}}=\gamma_{g_{22}}\left\{B_{g_{22}} s_{2}-\sigma_{22}\left(\hat{\theta}_{g_{22}}-\theta_{g_{22}}^{0}\right)\right\}, \\
& \dot{\hat{\theta}}_{g_{32}}=\gamma_{g_{32}}\left\{B_{g_{32}} s_{2}-\sigma_{32}\left(\hat{\theta}_{g_{32}}-\theta_{g_{32}}^{0}\right)\right\}, \\
& \dot{\hat{\theta}}_{g_{13}}=\gamma_{g_{13}}\left\{B_{g_{13}} s_{3}-\sigma_{13}\left(\hat{\theta}_{g_{13}}-\theta_{g_{13}}^{0}\right)\right\}, \\
& \dot{\hat{\theta}}_{g_{23}}=\gamma_{g_{23}}\left\{B_{g_{23}} s_{3}-\sigma_{23}\left(\hat{\theta}_{g_{23}}-\theta_{g_{23}}^{0}\right)\right\}, \\
& \dot{\hat{\theta}}_{g_{33}}=\gamma_{g_{33}}\left\{B_{g_{33}} s_{3}-\sigma_{33}\left(\hat{\theta}_{g_{33}}-\theta_{g_{33}}^{0}\right)\right\},
\end{aligned}
$$

where $\sigma>0$ is a leakage constant defined similar to the popular $\sigma$-modification [27] and is used to prevent the estimates from experiencing parameter drift. The constants $\gamma_{f_{i}}>0$ and $\gamma_{g_{i j}}>0$ represent the adaptation gains and $\theta_{f_{i}}^{0}$ are design parameters. To update the reconstruction error bounds $\hat{\eta}_{f f_{i}}$, we apply the following adaptive laws:

$$
\begin{aligned}
& \dot{\hat{\eta}}_{f_{1}}=\gamma_{f f_{1}}\left\{s_{1} \tanh \frac{s_{1}}{\epsilon}-\sigma_{1}\left(\hat{\eta}_{f f_{1}}-\eta_{f f_{1}}^{0}\right)\right\}, \\
& \dot{\hat{\eta}}_{f f_{2}}=\gamma_{f f_{2}}\left\{s_{2} \tanh \frac{s_{2}}{\epsilon}-\sigma_{2}\left(\hat{\eta}_{f f_{2}}-\eta_{f f_{2}}^{0}\right)\right\}, \\
& \dot{\hat{\eta}}_{f f_{3}}=\gamma_{f f_{3}}\left\{s_{3} \tanh \frac{s_{3}}{\epsilon}-\sigma_{3}\left(\hat{\eta}_{f f_{3}}-\eta_{f f_{3}}^{0}\right)\right\},
\end{aligned}
$$

where $\eta_{f f_{i}}^{0}$ are design parameters and $\epsilon>0$ is a small design constant used to smooth out the sign function by converting it into a hyperbolic function in this case tanh. These adaptive laws were derived based on the Lyapunov stability analysis to maintain system stability and prevent parameter drift. The control inputs in vector form are determined to be

$$
u=\left[\begin{array}{c}
\delta \\
F_{T} \\
M
\end{array}\right]=\hat{g}\left(x ; \hat{\theta}_{g}\right)^{-1} \hat{W}_{f}
$$

where $\hat{g}$ is a $3 x 3$ matrix that is a collection of the uncertain system functions from (5.69) and given by

$$
\hat{g}(x)=\left[\begin{array}{lll}
\hat{g}_{11}\left(x ; \hat{\theta}_{g_{11}}\right) & \hat{g}_{12}\left(x ; \hat{\theta}_{g_{12}}\right) & \hat{g}_{13}\left(x ; \hat{\theta}_{g_{13}}\right) \\
\hat{g}_{21}\left(x ; \hat{\theta}_{g_{21}}\right) & \hat{g}_{22}\left(x ; \hat{\theta}_{g_{22}}\right) & \hat{g}_{23}\left(x ; \hat{\theta}_{g_{23}}\right) \\
\hat{g}_{31}\left(x ; \hat{\theta}_{g_{31}}\right) & \hat{g}_{32}\left(x ; \hat{\theta}_{g_{32}}\right) & \hat{g}_{33}\left(x ; \hat{\theta}_{g_{33}}\right)
\end{array}\right]
$$


The matrix $\hat{W}_{f}$, is

$$
\hat{W}_{f}=\left[\begin{array}{c}
-\hat{\theta}_{f_{1}}^{T} B_{f_{1}}-\hat{\eta}_{f f_{1}} \tanh \frac{s_{1}}{\epsilon}+\dot{y}_{d_{1}}-c_{1} e_{1}-k_{1} s_{1}-\sum_{i=1}^{3} \eta_{f f_{1, i}} \operatorname{sgn}\left(s_{1}\right) \\
-\hat{\theta}_{f_{2}}^{T} B_{f_{2}}-\hat{\eta}_{f f_{2}} \tanh \frac{s_{2}}{\epsilon}+\dot{y}_{d_{2}}-c_{2} e_{2}-k_{2} s_{2}-\sum_{i=1}^{3} \eta_{f f_{2, i}} \operatorname{sgn}\left(s_{2}\right) \\
-\hat{\theta}_{f_{3}}^{T} B_{f_{3}}-\hat{\eta}_{f f_{3}} \tanh \frac{s_{3}}{\epsilon}+\dot{y}_{d_{3}}-c_{3} e_{3}-k_{3} s_{3}-\sum_{i=1}^{3} \eta_{f f_{3, i}} \operatorname{sgn}\left(s_{3}\right)
\end{array}\right],
$$

where $c_{i}$ and $k_{i}$ are positive real numbers. We have to mention restrictions that guarantee the existence of $\hat{g}\left(x ; \hat{\theta}_{g}\right)^{-1}$ is defined. The first of which is that the sign of the determinant of $\hat{g}$ remains constant over the range of possible variations of the function approximation and the next requirement is that each element of $\hat{g}$ are sign definite.

The stability analysis is verified using the Lyapunov method and is addressed by first defining the positive definite Lyaponov function as

$$
\begin{aligned}
V\left(s, \tilde{\theta}_{f_{i}}, \tilde{\theta}_{g_{i, j}}, \tilde{\eta}_{f f_{i}}\right)= & \frac{1}{2} s^{T} s+\sum_{i=1}^{3} \frac{\tilde{\theta}_{f_{i}}^{T} \tilde{\theta}_{f_{i}}}{2 \gamma_{f_{i}}}+\sum_{i=1}^{3} \frac{\tilde{\eta}_{f f_{i}}^{2}}{2 \gamma_{f f_{i}}}+ \\
& +\sum_{i=1}^{3} \sum_{j=1}^{3} \frac{\tilde{\theta}_{g_{i, j}}^{T} \tilde{\theta}_{g_{i, j}}}{2 \gamma_{g_{i, j}}}
\end{aligned}
$$

where $\gamma_{f_{i}}, \gamma_{g_{i, j}}$ and $\gamma_{f f_{i}}$ are the adaptive gains, $\tilde{\eta}_{f f_{i}}=\hat{\eta}_{f f_{i}}-\eta_{f f_{i}}^{*}$ the adaptive bounds estimation errors, for the case of $i=1,2,3$ and $j=1,2,3$.

As a measure of the systems error functions $s(t), \tilde{\theta}_{f_{i}}(t)$ and $\tilde{\theta}_{g_{i, j}}(t)$. Applying the fact that $\dot{\tilde{\theta}}_{f_{i}}(t)=\dot{\hat{\theta}}_{f_{i}}(t), \dot{\tilde{\theta}}_{g_{i, j}}(t)=\dot{\hat{\theta}}_{g_{i, j}}(t)$, and $\dot{\tilde{\eta}}_{f f_{i}}(t)=\dot{\hat{\eta}}_{f f_{i}}(t)$ we take the derivative of $V$ defined in equation (5.105) and inserting equations (5.69) and (5.88) as

$$
\begin{aligned}
\dot{V}=s^{T} & {\left[\begin{array}{c}
c_{1} e_{1}-\dot{y}_{d_{1}} \\
c_{2} e_{2}-\dot{y}_{d_{2}} \\
c_{3} e_{3}-\dot{y}_{d_{3}}
\end{array}\right]+s^{T}\{f+g u\}+\sum_{i=1}^{3} \frac{\tilde{\theta}_{f_{i}}^{T} \dot{\hat{\theta}}_{f_{i}}}{\gamma_{f_{i}}}+} \\
& +\sum_{i=1}^{3} \sum_{j=1}^{3} \frac{\tilde{\theta}_{g_{i, j}}^{T} \dot{\hat{\theta}}_{g_{i, j}}}{\gamma_{g_{i, j}}}+\sum_{i=1}^{3} \frac{\tilde{\eta}_{f f_{i}} \dot{\hat{\eta}}_{f f_{i}}}{\gamma_{f f_{i}}} .
\end{aligned}
$$

Next, we add and subtract the terms $s^{T} \hat{f}$ and $s^{T} \hat{g} u$ to be inserted into equation (5.106) as

$$
\dot{V}=s^{T}\left\{\left[\begin{array}{c}
c_{1} e_{1}-\dot{y}_{d_{1}} \\
c_{2} e_{2}-\dot{y}_{d_{2}} \\
c_{3} e_{3}-\dot{y}_{d_{3}}
\end{array}\right]+\left[\begin{array}{c}
\eta_{f_{1}} \\
\eta_{f_{2}} \\
\eta_{f_{3}}
\end{array}\right]-\left[\begin{array}{c}
\hat{\theta}_{f_{1}}^{T} B_{f_{1}} \\
\hat{\theta}_{f_{2}}^{T} B_{f_{2}} \\
\hat{\theta}_{f_{3}}^{T} B_{f_{3}}
\end{array}\right]+\hat{f}+\right.
$$




$$
\begin{aligned}
& +\hat{g} u-\left[\begin{array}{llll}
\tilde{\theta}_{g_{1,1}}^{T} B_{g_{1,1}} & \tilde{\theta}_{g_{2,1}}^{T} B_{g_{2,1}} & \tilde{\theta}_{g_{3,1}}^{T} B_{g_{3,1}} \\
\tilde{\theta}_{g_{1,2}}^{T} B_{g_{1,2}} & \tilde{\theta}_{g_{2,2}}^{T} B_{g_{2,2}} & \tilde{\theta}_{g_{3,2}}^{T} B_{g_{3,2}} \\
\tilde{\theta}_{g_{1,3}}^{T} B_{g_{1,3}} & \tilde{\theta}_{g_{2,3}}^{T} B_{g_{2,3}} & \tilde{\theta}_{g_{3,3}}^{T} B_{g_{3,3}}
\end{array}\right] u+ \\
& \left.+\left[\begin{array}{ccc}
\eta_{g_{1,1}} & \eta_{g_{2,1}} & \eta_{g_{3,1}} \\
\eta_{g_{1,2}} & \eta_{g_{2,2}} & \eta_{g_{3,2}} \\
\eta_{g_{1,3}} & \eta_{g_{2,3}} & \eta_{g_{3,3}}
\end{array}\right]\left[\begin{array}{l}
1 \\
1 \\
1
\end{array}\right]\right\}+\sum_{i=1}^{3} \frac{\tilde{\theta}_{f_{i}}^{T} \dot{\hat{\theta}}_{f_{i}}}{\gamma_{f_{i}}}+ \\
& +\sum_{i=1}^{3} \sum_{j=1}^{3} \frac{\tilde{\theta}_{g_{i, j}}^{T} \dot{\hat{\theta}}_{g_{i, j}}}{\gamma_{g_{i, j}}}+\sum_{i=1}^{3} \frac{\tilde{\eta}_{f f_{i}} \dot{\hat{\eta}}_{f f_{i}}}{\gamma_{f f_{i}}} .
\end{aligned}
$$

Implementing the reconstruction error bounds and plugging in the parameter update laws from equations (5.89) - (5.100) into the equation (5.107) we obtain

$$
\begin{gathered}
\dot{V} \leq s^{T}\left\{\left[\begin{array}{c}
c_{1} e_{1}-\dot{y}_{d_{1}} \\
c_{2} e_{2}-\dot{y}_{d_{2}} \\
c_{3} e_{3}-\dot{y}_{d_{3}}
\end{array}\right]+\left[\begin{array}{lll}
\eta_{g_{1,1}} & \eta_{g_{2,1}} & \eta_{g_{3,1}} \\
\eta_{g_{1,2}} & \eta_{g_{2,2}} & \eta_{g_{3,2}} \\
\eta_{g_{1,3}} & \eta_{g_{2,3}} & \eta_{g_{3,3}}
\end{array}\right]\left[\begin{array}{l}
1 \\
1 \\
1
\end{array}\right]+\hat{f}+\hat{g} u\right\}+ \\
+\|s\|^{T}\left[\begin{array}{c}
\eta_{f f_{1}}^{*} \\
\eta_{f f_{2}}^{*} \\
\eta_{f f_{3}}^{*}
\end{array}\right]+\sum_{i=1}^{3} \frac{\tilde{\eta}_{f f_{i}} \dot{\hat{\eta}}_{f f_{i}}}{\gamma_{f f_{i}}}-\left[\sigma_{1} \sigma_{2} \sigma_{3}\right]\left[\begin{array}{c}
\hat{\theta}_{f_{1}}^{T}\left(\hat{\theta}_{f_{1}}^{T}-\theta_{f_{1}}^{0}\right) \\
\hat{\theta}_{f_{2}}^{T}\left(\hat{\theta}_{f_{2}}^{T}-\theta_{f_{2}}^{0}\right) \\
\hat{\theta}_{f_{3}}^{T}\left(\hat{\theta}_{f_{3}}^{T}-\theta_{f_{3}}^{0}\right)
\end{array}\right],
\end{gathered}
$$

where $\|s\|=\left[\left\|s_{1}\right\|\left\|s_{2}\right\|\left\|s_{3}\right\|\right]^{T}$. We then substitute in the control inputs defined in equation (5.104) to yield

$$
\begin{gathered}
\dot{V} \leq\|s\|^{T}\left[\begin{array}{c}
\eta_{f f_{1}}^{*} \\
\eta_{f f_{2}}^{*} \\
\eta_{f f_{3}}^{*}
\end{array}\right]-s^{T}\left[\begin{array}{l}
\left\{\hat{\eta}_{f f_{1}} \tanh \left(\frac{s_{1}}{\epsilon}\right)+k_{1} s_{1}\right\} \\
\left\{\hat{\eta}_{f f_{2}} \tanh \left(\frac{s_{2}}{\epsilon}\right)+k_{2} s_{2}\right\} \\
\left\{\hat{\eta}_{f f_{3}} \tanh \left(\frac{s_{3}}{\epsilon}\right)+k_{3} s_{3}\right\}
\end{array}\right]- \\
-\left[\sigma_{1} \sigma_{2} \sigma_{3}\right]\left[\begin{array}{c}
\tilde{\theta}_{f_{1}}^{T}\left(\hat{\theta}_{f_{1}}-\theta_{f_{1}}^{0}\right) \\
\tilde{\theta}_{f_{2}}^{T}\left(\hat{\theta}_{f_{2}}-\theta_{f_{2}}^{0}\right) \\
\tilde{\theta}_{f_{3}}^{T}\left(\hat{\theta}_{f_{3}}-\theta_{f_{3}}^{0}\right)
\end{array}\right]+\sum_{i=1}^{3} \frac{\tilde{\eta}_{f f_{i}} \dot{\hat{\eta}}_{f f_{i}}}{\gamma_{f f_{i}}} .
\end{gathered}
$$

Using the hyperbolic tangent property defined in [48], (5.101), (5.102) and (5.103) we reduce the derivative of $V(5.109)$ as

$$
\dot{V} \leq-k s^{T} s+\eta_{f f_{1}}^{*} \kappa \epsilon+\eta_{f f_{2}}^{*} \kappa \epsilon+\eta_{f f_{3}}^{*} \kappa \epsilon-
$$




$$
-\left[\sigma_{1} \sigma_{2} \sigma_{3}\right]\left[\begin{array}{l}
\tilde{\theta}_{f_{1}}^{T}\left(\hat{\theta}_{f_{1}}-\theta_{f_{1}}^{0}\right)+\tilde{\eta}_{f f_{1}}^{T}\left(\hat{\eta}_{f f_{1}}-\eta_{f f_{1}}^{0}\right) \\
\tilde{\theta}_{f_{2}}^{T}\left(\hat{\theta}_{f_{2}}-\theta_{f_{2}}^{0}\right)+\tilde{\eta}_{f f_{2}}^{T}\left(\hat{\eta}_{f f_{2}}-\eta_{f f_{2}}^{0}\right) \\
\tilde{\theta}_{f_{3}}^{T}\left(\hat{\theta}_{f_{3}}-\theta_{f_{3}}^{0}\right)+\tilde{\eta}_{f f_{3}}^{T}\left(\hat{\eta}_{f f_{3}}-\eta_{f f_{3}}^{0}\right)
\end{array}\right] .
$$

Following the stability analysis from [50] we conclude that $\dot{V}$ is not negative definite, therefore asymptotic convergence is not guaranteed. However, for a bounded set, the solution $V$, can be shown to conclude system tracking errors, parameters and adaptive bounds are uniformly ultimately bounded for a well defined compact set $\Omega$.

\subsubsection{Space Flight Control Performance Evaluation}

The spline function based adaptive control laws designed in the previous section are implemented here to a realistic spacecraft system with physical parameters. The control objective is to track the desired output signals and maintain system stability during an orbital transfer. The physical parameters used in the simulations are $m=480 \mathrm{~kg}, I=400 \mathrm{~kg} \cdot \mathrm{m}^{2}, m_{f}=$ $50 \mathrm{~kg}, I_{f}=10 \mathrm{~kg} \cdot \mathrm{m}^{2}, b=1.5 \mathrm{~m}, d=1.25 \mathrm{~m}, a=0.21 \mathrm{~m}, \mathrm{~F}=2300 \mathrm{~N}$, and $\epsilon=3.7 \mathrm{~kg} \cdot \mathrm{m}^{2} / \mathrm{s}$, as given in [53].

Simulation. In this section, we illustrate the performance of our approximation based adaptive controller design applied to the complete nonlinear system

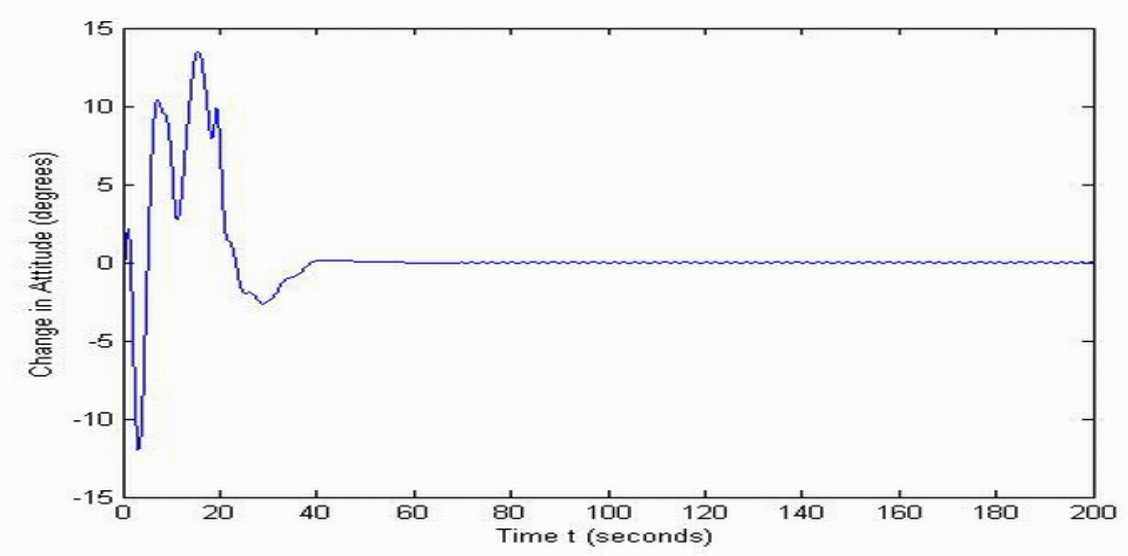

Figure 5.7: Tracking error $y(t)-y_{d}(t)$ for the reduced order MIMO spacecraft model with fuel slosh. 


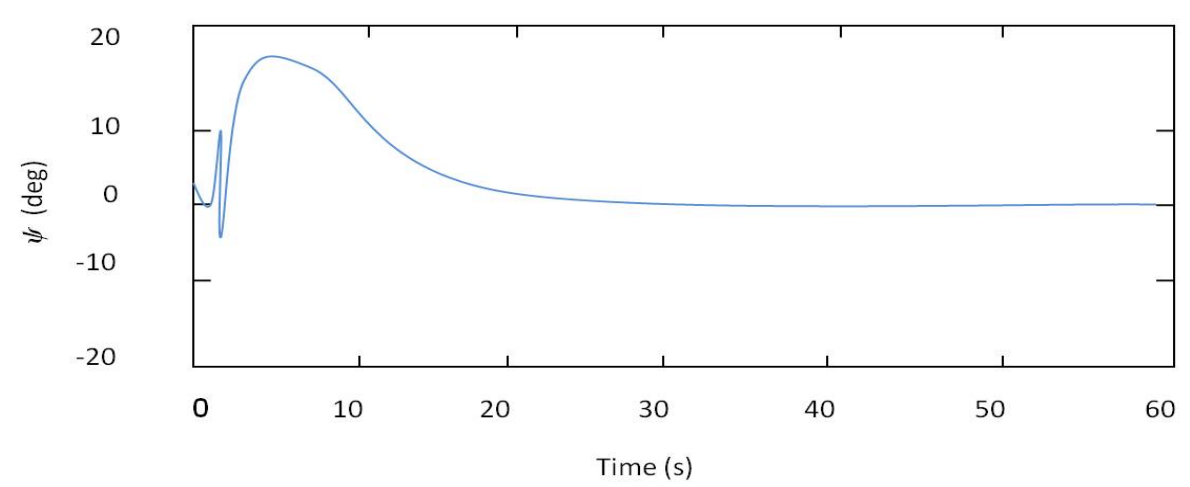

Figure 5.8: Slosh Angle $\psi$ Time Response.

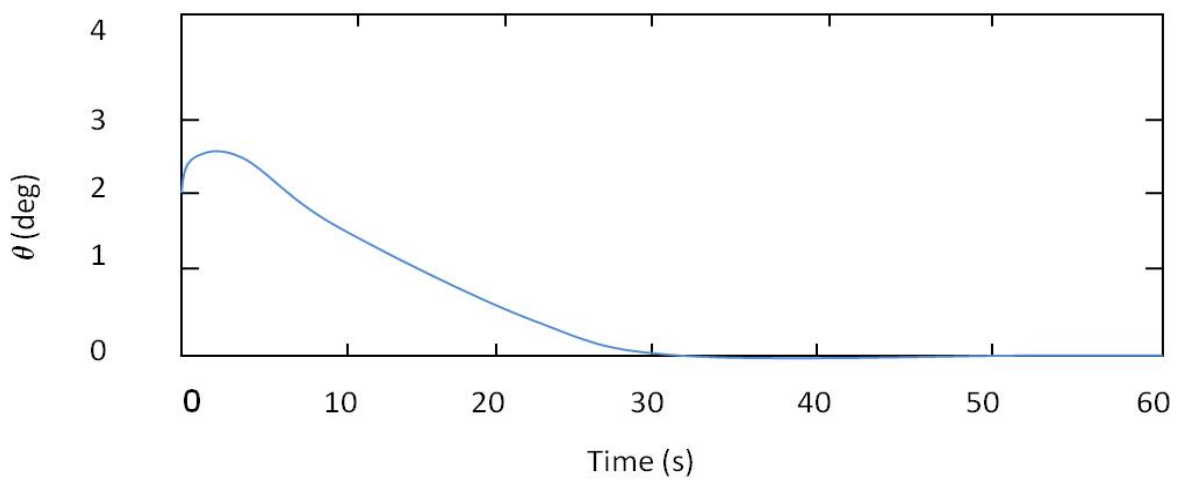

Figure 5.9: Attitude Angle $\theta$ : Output Time Response.

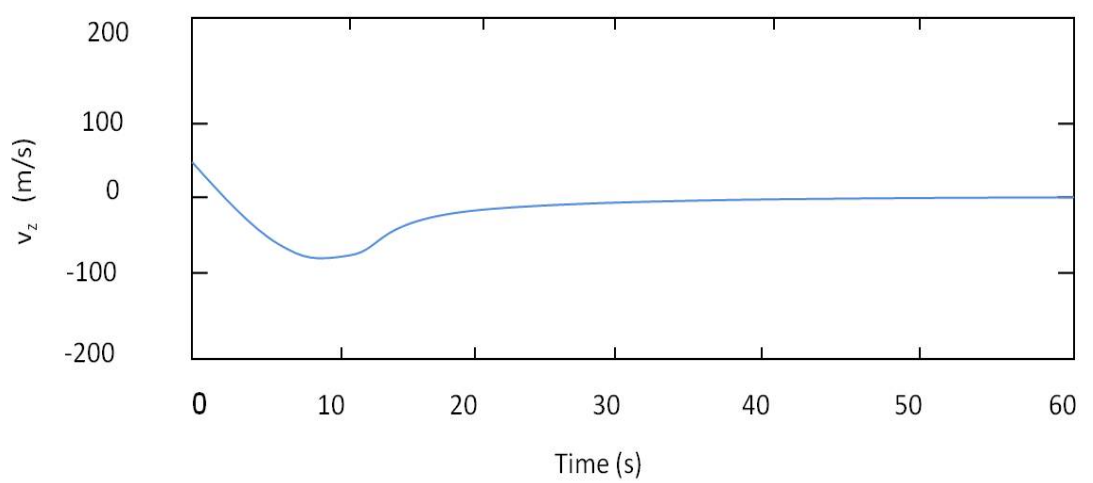

Figure 5.10: Transverse Velocity $v_{z}$ : Output Time Response. 
Where the corresponding control inputs are
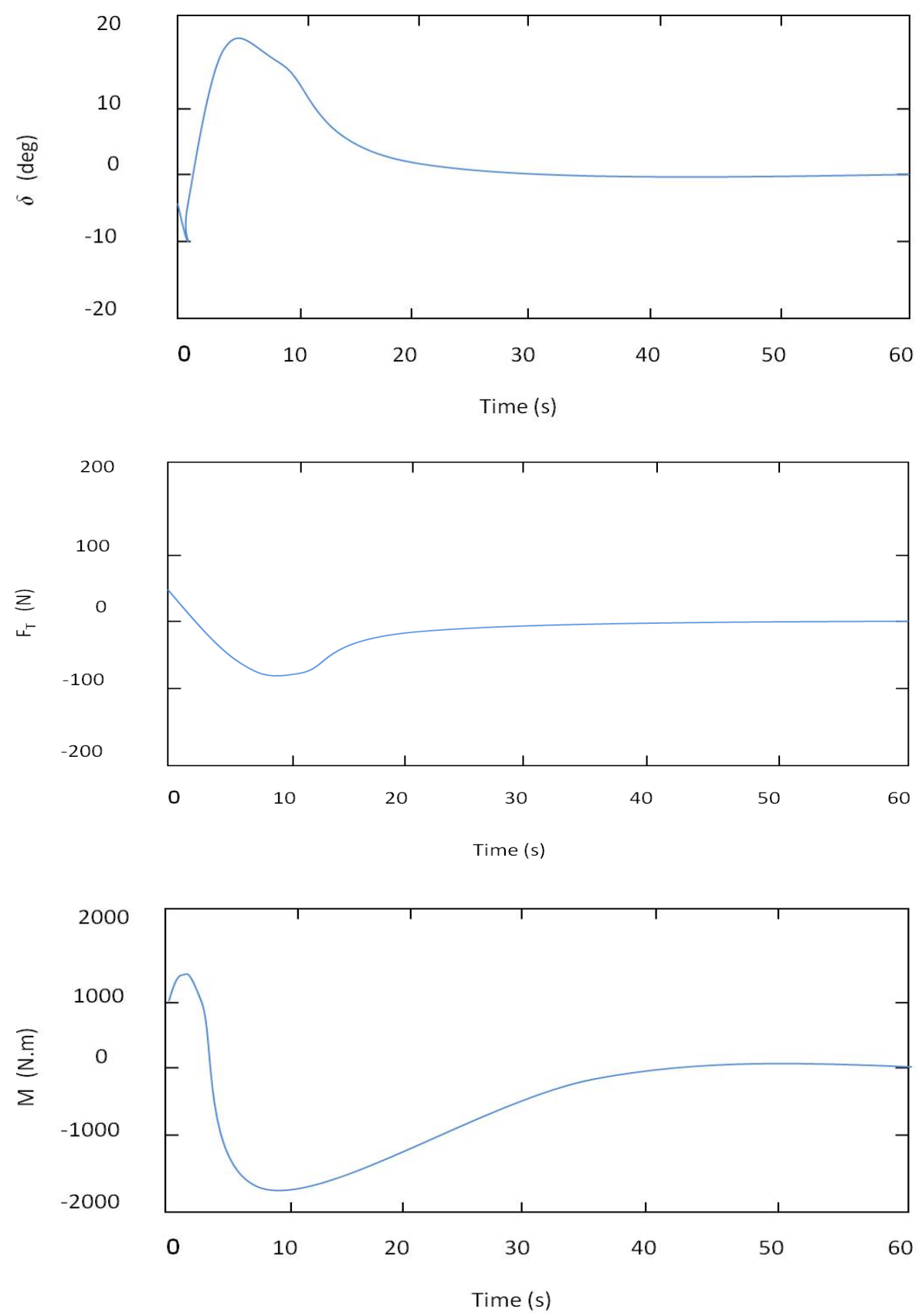

Figure 5.11: Transverse Control Force $F_{T}$, Pitching Moment $M$, and Gimbal Deflection Angle $\delta$.

Here we have developed a MIMO nonlinear dynamical model for a spacecraft with a single fuel sloshing mode. Where the spline function based adaptive control design scheme 
achieves bounding tracking for a set of desired output signals. The effectiveness of our approximation based design has been illustrated by a number of different simulation conditions. We anticipate our future research to include the adaptive control design and system dynamics of a spacecraft with multiple fuel slosh modes. 


\section{Chapter 6}

\section{Adaptive Compensation of}

\section{Synthetic Jet Actuator}

\section{Nonlinearities}

Recall the $i^{\text {th }}$ synthetic jet actuator nonlinearity model with uncertain parameters as defined earlier is described by

$$
u_{i}(t)=N_{i}\left(\theta_{i}^{*} ; v_{i}, \alpha\right)
$$

where $u_{i}(t)$ is the $i^{t h}$ control input to the plant and is generated by synthetic jet actuators, $i=1,2, \ldots, m$, where $m>1$ is the number of control inputs, $v_{i}(t)$ is the input to each actuator, $\alpha$ is the angle of attack, and $\theta_{i}^{*}$ is the unknown parameter vector. It is important to note that for a realistic study the actuator output $u_{i}(t)$ is not available for measurement. In this case, an approximation is a good choice.

To overcome these challenges, we develop a linearly parametrized spline function approximation for each $i^{\text {th }}$ function denoted $f_{i}\left(v_{i}, \alpha\right)$. That is, the nonlinearity profile can be approximated by multivariable B-splines defined as

$$
u_{i}(t)=N_{i}\left(\theta_{N_{i}}^{*} ; v_{i}, \alpha\right) \triangleq f_{i}\left(v_{i}, \alpha\right)=\theta_{N_{i}}^{* T} B_{N_{i}}\left(v_{i}, \alpha\right)+\eta_{N_{i}}\left(v_{i}, \alpha\right),
$$

where the expression is,

$$
\theta_{N_{i}}^{* T} B_{N_{i}}\left(v_{i}, \alpha\right)=\sum_{j_{1}=1}^{M_{1}} \sum_{j_{2}=1}^{M_{2}} \theta_{j_{1} j_{2}}^{*} b_{j_{1}}(v) b_{j_{2}}(\alpha),
$$


$\eta_{N_{i}}$ represents the $i^{\text {th }}$ spline function approximation error and is bounded by a positive constant $\left\|\eta_{N_{i}}\right\| \leq \eta_{N N_{i}}, \theta_{j_{1} j_{2}}^{*}$ for each $i^{t h}$ approximator is one of $\left(M_{1} \cdot M_{2}\right)$ unknown Bspline coefficients, and $b_{j_{1}}(v), b_{j_{2}}(\alpha)$ are the univariable B-spline basis elements, and the estimator of the synthetic jet actuator nonlinearity function as

$$
\hat{u}_{i}(t)=\hat{N}_{i}\left(\hat{\theta}_{N_{i}} ; v_{i}, \alpha\right)=\hat{\theta}_{N_{i}}^{T} B_{N_{i}}\left(v_{i}, \alpha\right)
$$

Smooth Adaptive Inverse Design. For the nonlinearity function $N_{i}\left(u_{d i}, \alpha\right)$, we assume it is invertible and continuous such that there exists $N I_{i}\left(u_{d}, \alpha\right)$ to ensure that $u_{i}(t)=N_{i}\left(N I_{i}\left(u_{d_{i}}, \alpha\right), \alpha\right)=u_{d_{i}}(t)$ the inputs to the aircraft dynamics. With these assumptions we can express the nonlinearity inverse function $N I_{i}\left(u_{d_{i}}, \alpha\right)$, in the following form:

$$
N I_{i}\left(u_{d_{i}}, \alpha\right)=f_{i}^{-1}\left(u_{d_{i}}, \alpha\right)=u_{d S_{i}}
$$

where $f_{i}^{-1}\left(u_{d_{i}}, \alpha\right)$ is the inverse function for the synthetic jet actuator nonlinearity, $u_{d_{i}}$, as mentioned before, is the desired input signal. In our previous research [66], we consider the case where the function $u_{d S_{i}}$ may be discontinuous, for example actuator nonlinearities that exhibit a deadzone or backlash behavior.

Based on the spline function network property, we can approximate the $i^{\text {th }}$ nonlinearity inverse function by

$$
u_{d S_{i}}=N I_{i}\left(\theta_{s_{i}}^{*} ; u_{d_{i}}, \alpha\right) \triangleq \theta_{s_{i}}^{* T} B_{s_{i}}\left(u_{d_{i}}, \alpha\right)+\eta_{s_{i}}\left(u_{d_{i}}, \alpha\right),
$$

where

$$
\theta_{s_{i}}^{* T} B_{s_{i}}\left(u_{d_{i}}, \alpha\right)=\sum_{j_{1}=1}^{R_{1}} \sum_{j_{2}=1}^{R_{2}} \theta_{j_{1} j_{2}}^{*} b_{j_{1}}\left(u_{d}\right) b_{j_{2}}(\alpha),
$$

$\eta_{s_{i}}$ represents the $i^{t h}$ spline function network approximation error and is bounded by a positive constant as $\left\|\eta_{s_{i}}\right\| \leq \eta_{N S_{i}}, \theta_{j_{1} j_{2}}^{*}$ for each $i^{t h}$ approximator is one of $\left(R_{1} \cdot R_{2}\right)$ unknown B-spline coefficients, $b_{j_{1}}\left(u_{d}\right), b_{j_{2}}(\alpha)$ are the univariable B-spline basis elements. Next, we define the estimates of the synthetic jet actuator nonlinearity inverse functions as

$$
\hat{u}_{d S_{i}}(t)=\widehat{N I}_{i}\left(\hat{\theta}_{s_{i}} ; u_{d_{i}}, \alpha\right)=\hat{\theta}_{s_{i}}^{T} B_{s_{i}}\left(u_{d_{i}}, \alpha\right)
$$

Applying (6.5) and the estimation error $\tilde{u}_{d S_{i}}=u_{d S_{i}}-\hat{u}_{d S_{i}}$, we obtain

$$
u_{d_{i}}=f_{i}\left(\hat{u}_{d S_{i}}+\tilde{u}_{d S_{i}}, \alpha\right)
$$




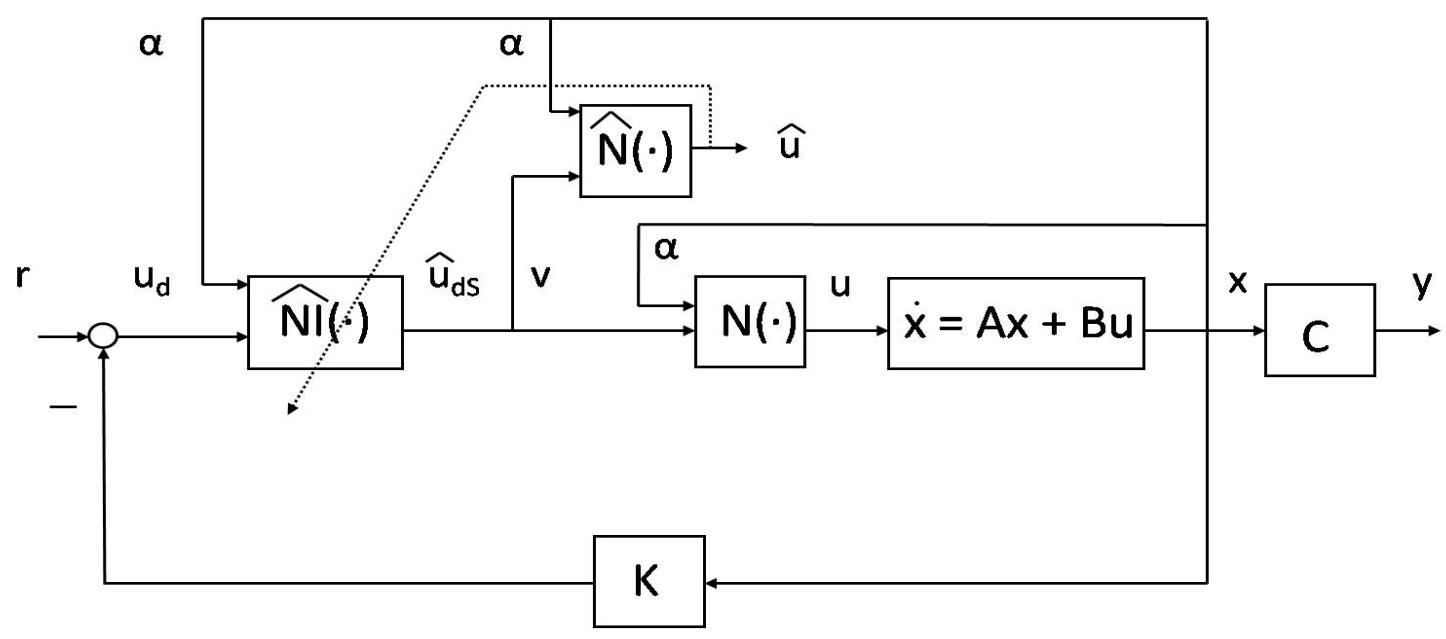

Figure 6.1: Approximation based smooth adaptive inverse compensation control.

Following from equation (6.5), we define the applied control inputs as (see Figure 6.1)

$$
v_{i}=\hat{u}_{d S_{i}}
$$

now we can take the Taylor series expansion of (6.9) about $u_{d S_{i}}=\hat{u}_{d S_{i}}$, to obtain the desired control signals $u_{d_{i}}$ as

$$
u_{d_{i}}=f_{i}\left(v_{i}, \alpha\right)+\frac{\partial f_{i}\left(v_{i}, \alpha\right)}{\partial v_{i}} \tilde{u}_{d S_{i}}+O\left(\frac{\partial f_{i}^{l}\left(v_{i}, \alpha\right)}{\partial v_{i}^{l}}, \tilde{u}_{d S_{i}}\right)
$$

where $l=2,3, \ldots, \infty$ and $O(\cdot)$ represents the higher order derivative terms in the Taylor series expansion. The representation of $u_{d_{i}}(t)$ in (6.11) is written in this form to allow the derivation of each $i^{\text {th }}$ control error $u_{i}(t)-u_{d_{i}}(t)$. The full state desired linear feedback control law is defined as $u_{d}(t)=r(t)-K x(t)$, where $u_{d}(t)=\left[u_{d_{1}}, u_{d_{2}}, \ldots, u_{d_{m}}\right] \in \Re^{m},($ recall that $m>1$ is the number of control inputs and $i=1,2, \ldots, m)$, and $K$ is chosen such that the poles of the solution $A-B K$ are equal to some desired closed loop poles. Figure 6.1 describes an adaptive state feedback inverse control system for smooth nonlinearities that contains two spline function network approximations. The first approximation structure is used as a compensator for the inverse nonlinearity, while the second structure is an estimator for the uncertain synthetic jet actuator nonlinearity. 


\subsection{Control Error}

Next, we derive an expression for the $i^{t h}$ control error $u_{i}(t)-u_{d_{i}}(t)$ to describe the effectiveness of the nonlinearity functional approximations. This expression is critical in developing adaptive update laws for the parameter estimates.

Lemma 1 : With the functional compensator described by (6.8), (6.10) and the estimate represented by (6.4), the $i^{\text {th }}$ control error for the spline function-based synthetic jet actuator nonlinearity compensation scheme is given by

$$
u_{i}(t)-u_{d_{i}}(t)=\tilde{\theta}_{N_{i}}^{T} \frac{\partial B_{N_{i}}\left(v_{i}, \alpha\right)}{\partial v_{i}} \hat{\theta}_{s_{i}}^{T} B_{s_{i}}\left(u_{d_{i}}, \alpha\right)-\hat{\theta}_{N_{i}}^{T} \frac{\partial B_{N_{i}}\left(v_{i}, \alpha\right)}{\partial v_{i}} \tilde{\theta}_{s_{i}}^{T} B_{s_{i}}\left(u_{d_{i}}, \alpha\right)+\eta_{i}(t)
$$

where $\eta_{i}(t)$ is the model mismatch error.

Proof: From (6.2) and (6.11), the control error is given as

$$
u_{i}-u_{d_{i}}=-\frac{\partial f_{i}\left(v_{i}, \alpha\right)}{\partial v_{i}} \tilde{u}_{d S_{i}}-O\left(\frac{\partial f_{i}^{l}\left(v_{i}, \alpha\right)}{\partial v_{i}^{l}}, \tilde{u}_{d S_{i}}\right) .
$$

Substituting (6.6) and (6.8) in (6.13), we obtain

$$
u_{i}-u_{d_{i}}=\frac{\partial f_{i}\left(v_{i}, \alpha\right)}{\partial v_{i}} \tilde{\theta}_{s_{i}}^{T} B_{s_{i}}\left(u_{d_{i}}, \alpha\right)-\frac{\partial f_{i}\left(v_{i}, \alpha\right)}{\partial v_{i}} \eta_{s_{i}}\left(u_{d_{i}}, \alpha\right)-O\left(\frac{\partial f_{i}^{l}\left(v_{i}, \alpha\right)}{\partial v_{i}^{l}}, \tilde{u}_{d S_{i}}\right)
$$

where $\tilde{\theta}_{s_{i}}(t)=\hat{\theta}_{s_{i}}(t)-\theta_{s_{i}}^{*}$ is the parameter error for the $i^{t h}$ inverse nonlinearity function. From $(6.2),(6.4),(6.14)$ and $\tilde{\theta}_{N_{i}}(t)=\hat{\theta}_{N_{i}}(t)-\theta_{N_{i}}^{*}$ the parameter error for the $i^{t h}$ nonlinearity function, this results in the following form

$$
\begin{aligned}
u_{i}-u_{d_{i}}= & \left(\frac{\partial \eta_{N_{i}}\left(v_{i}, \alpha\right)}{\partial v_{i}}-\tilde{\theta}_{N_{i}}^{T} \frac{\partial B_{N_{i}}\left(v_{i}, \alpha\right)}{\partial v_{i}}\right) \tilde{\theta}_{s_{i}}^{T} B_{s_{i}}\left(u_{d_{i}}, \alpha\right)-\frac{\partial f_{i}\left(v_{i}, \alpha\right)}{\partial v_{i}} \eta_{s_{i}}\left(u_{d_{i}}, \alpha\right)- \\
& -O\left(\frac{\partial f_{i}^{l}\left(v_{i}, \alpha\right)}{\partial v_{i}^{l}}, \tilde{u}_{d S_{i}}\right)+\hat{\theta}_{N_{i}}^{T} \frac{\partial B_{N_{i}}\left(v_{i}, \alpha\right)}{\partial v_{i}} \tilde{\theta}_{s_{i}}^{T} B_{s_{i}}\left(u_{d_{i}}, \alpha\right) \\
= & -\tilde{\theta}_{N_{i}}^{T} \frac{\partial B_{N_{i}}\left(v_{i}, \alpha\right)}{\partial v_{i}} \hat{\theta}_{s_{i}}^{T} B_{s_{i}}\left(u_{d_{i}}, \alpha\right)+\hat{\theta}_{N_{i}}^{T} \frac{\partial B_{N_{i}}\left(v_{i}, \alpha\right)}{\partial v_{i}} \tilde{\theta}_{s_{i}}^{T} B_{s_{i}}\left(u_{d_{i}}, \alpha\right)+\eta_{i},
\end{aligned}
$$

where $\eta_{i}(t)$ is

$$
\begin{aligned}
\eta_{i}= & \tilde{\theta}_{N_{i}}^{T} \frac{\partial B_{N_{i}}\left(v_{i}, \alpha\right)}{\partial v_{i}} \theta_{s_{i}}^{* T} B_{s_{i}}\left(u_{d_{i}}, \alpha\right)+\frac{\partial \eta_{N_{i}}\left(v_{i}, \alpha\right)}{\partial v_{i}} \tilde{\theta}_{s_{i}}^{T} B_{s_{i}}\left(u_{d_{i}}, \alpha\right)-O\left(\frac{\partial f_{i}^{l}\left(v_{i}, \alpha\right)}{\partial v_{i}^{l}}, \tilde{u}_{d S_{i}}\right) \\
& -\theta_{N_{i}}^{* T} \frac{\partial B_{N_{i}}\left(v_{i}, \alpha\right)}{\partial v_{i}} \eta_{s_{i}}\left(u_{d_{i}}, \alpha\right) .
\end{aligned}
$$


This expresses the $i^{t h}$ control error $u_{i}(t)-u_{d_{i}}(t)$ in terms of the parameter errors $\tilde{\theta}_{N_{i}}(t)$ and $\tilde{\theta}_{s_{i}}(t)$. Note that the form of $(6.12)$ is crucial in controller design and in deriving adaptive laws that guarantee closed-loop stability. Also we must note that the modeling mismatch term $\eta_{i}(t)$ consists of approximation error terms and higher-order derivative terms from the Taylor series expansion performed earlier. The following important result gives the upper bound of the norm of $\eta_{i}(t)$ and is used in the stability proof, where $\|\cdot\|$ is used as any suitable vector norm.

Lemma 2 : The norm of the modeling mismatch term $\eta_{i}(t)$ in (6.12) is bounded by

$$
\left\|\eta_{i}(t)\right\| \leq \beta_{i}^{T} \Omega_{i}
$$

where $\beta_{i}=\left[\beta_{1_{i}}, \beta_{2_{i}}, \beta_{3_{i}}, \beta_{4_{i}},\right]^{T}$ is the $i^{\text {th }}$ unknown constant vector, being composed of bounded constants and the known $i^{\text {th }}$ vector function is $\Omega_{i}=\left[1,\left\|\hat{\theta}_{N_{i}}\right\|,\left\|\hat{\theta}_{s_{i}}\right\|,\left\|\hat{\theta}_{s_{i}}\right\|^{2}\right]^{T}$.

Proof: From (6.16) and the fact that there obviously exists the $i^{\text {th }}$ positive constants $\theta_{M_{i}}$ and $\theta_{S_{i}}$ satisfying $\left\|\theta_{N_{i}}^{*}\right\| \leq \theta_{M_{i}}$ and $\left\|\theta_{s_{i}}^{*}\right\| \leq \theta_{S_{i}}$, where $\theta_{M_{i}}$ and $\theta_{S_{i}}$ are not needed to be known. Based on the following facts for the bounds

$$
\left\|\tilde{\theta}_{N_{i}}\right\| \leq \theta_{M_{i}}+\left\|\hat{\theta}_{N_{i}}\right\|,\left\|\tilde{\theta}_{s_{i}}\right\| \leq \theta_{S_{i}}+\left\|\hat{\theta}_{s_{i}}\right\|
$$

we have each $i^{\text {th }}$ modeling mismatch error bounded by

$$
\begin{aligned}
\left\|\eta_{i}(t)\right\| \leq & \theta_{s_{i}}\left(\theta_{M_{i}}+\left\|\hat{\theta}_{N_{i}}\right\|\right)\left\|\frac{\partial B_{N_{i}}\left(v_{i}, \alpha\right)}{\partial v_{i}}\right\|\left\|B_{s_{i}}\left(u_{d_{i}}, \alpha\right)\right\|+\theta_{M_{i}}\left\|\frac{\partial B_{N_{i}}\left(v_{i}, \alpha\right)}{\partial v_{i}}\right\| \eta_{S_{i}}+\|O(\cdot)\| \\
& +\left\|\frac{\partial \eta_{N_{i}}\left(v_{i}, \alpha\right)}{\partial v_{i}}\right\|\left(\theta_{S_{i}}+\left\|\hat{\theta}_{s_{i}}\right\|\right)\left\|B_{s_{i}}\left(u_{d_{i}}, \alpha\right)\right\| .
\end{aligned}
$$

With some algebraic simplifications, this becomes

$$
\left\|\eta_{i}(t)\right\| \leq \beta_{1_{i}}+\beta_{2_{i}}\left\|\hat{\theta}_{N_{i}}\right\|+\beta_{3_{i}}\left\|\hat{\theta}_{s_{i}}\right\|+\beta_{4_{i}}\left\|\hat{\theta}_{s_{i}}\right\|^{2}=\beta_{i}^{T} \Omega_{i}
$$

The $i^{\text {th }}$ vector $\beta_{i}$ is concluded to be bounded because $\left\|B_{s_{i}}\left(u_{d_{i}}, \alpha\right)\right\|$ and $\left\|\frac{\partial B_{N_{i}}\left(v_{i}, \alpha\right)}{\partial v_{i}}\right\|$ are 
bounded for a bounded $\alpha$. Note that the $i^{\text {th }}$ control error (6.12) reflects the mutual dependence of the two parametrized nonlinearity functions. To proceed, we define an estimator for the bound of the $i^{\text {th }}$ model mismatch error $\beta_{i}^{T} \Omega_{i}$, as

$$
\hat{\eta}_{i}(t)=\hat{\beta}_{i}^{T} \Omega_{i}
$$

where $\hat{\beta}_{i}(t)=\left[\hat{\beta}_{1_{i}}, \hat{\beta}_{2_{i}}, \hat{\beta}_{3_{i}}, \hat{\beta}_{4_{i}}\right]^{T}$, to be updated from an adaptive law. A similar development is given in [85] with a neural network framework for discontinuous nonlinearities. $\nabla$

As the $i^{t h}$ estimates $\hat{\theta}_{N_{i}}(t)$ and $\hat{\theta}_{s_{i}}(t)$ approach the actual parameters $\theta_{N_{i}}^{*}$ and $\theta_{s_{i}}^{*}$, each spline function approximator effectively provides an inverse for the synthetic jet actuator nonlinearity. Observing the form of the control error expression (6.12) is critical for adaptive controller design, so as to guarantee closed-loop stability. Through the first-order Taylor expansion, the $i^{t h}$ control error $u_{i}(t)-u_{d_{i}}(t)$ has been expressed conveniently in a linearly parameterizable form with respect to the parameter errors $\tilde{\theta}_{N_{i}}(t)$ and $\tilde{\theta}_{s_{i}}(t)$. This allows us to adaptively update the estimates $\hat{\theta}_{N_{i}}(t)$ and $\hat{\theta}_{s_{i}}(t)$. Moreover, the mismatch error term $\eta_{i}(t)$ is bounded by a constant vector multiplied by a known function vector. Indeed, adaptive control techniques can be employed to handle these residual terms in a robust fashion.

The interesting fact that the modification of one nonlinearity function depends on the other, leads us to conclude that the adaptive update laws to be developed for $\hat{\theta}_{N_{i}}(t)$ and $\hat{\theta}_{s_{i}}(t)$ should be mutually coupled, such that the parameter errors $\tilde{\theta}_{N_{i}}(t)$ and $\tilde{\theta}_{s_{i}}(t)$ are bounded and the closed-loop system stability is guaranteed.

\subsection{Adaptive Feedback Control System}

In this section we present a state feedback adaptive inverse compensation scheme to cancel the $i^{\text {th }}$ control input nonlinearity $N_{i}\left(\theta_{i}^{*} ; v_{i}, \alpha\right)$, in order to ensure the system objective is achieved. Such a control system is that shown in Figure 6.1 (note that the output $y(t)$ is not used in this study, but the control scheme to be developed can be made for output tracking). 


\subsubsection{State Feedback Control}

Recall the m-input linearized time-invariant plant model for the commercial aircraft flight dynamics with synthetic jet actuators included,

$$
\begin{gathered}
\dot{x}(t)=A x(t)+B u(t)=A x(t)+\sum_{i=1}^{m} B_{i} u_{i}, \\
y(t)=C x(t), t \geq 0,
\end{gathered}
$$

where the angle of attack $\alpha$ is one of the components of the state vector $x(t) \in \Re^{n}, u(t) \in \Re^{m}$ is the control input, $y(t) \in \Re$ is the output, and $A \in \Re^{n \times n}, B \in \Re^{n \times m}$ and $C \in \Re^{1 \times n}$ are known constant parameter matrices. Here $B_{i}$ is defined as the $i^{\text {th }}$ column of $B$ for $i=1, \ldots, m$, such that $u_{i}(t)$ is the $i^{t h}$ element of $u(t)$ that is implemented with the synthetic jet actuators. To compensate for the approximation error uncertainty we modify the desired state feedback control signal as

$$
u_{d}(t)=-K x(t)+r(t)+v_{\eta}(t)
$$

where $u_{d}(t)=\left[u_{d_{1}}, u_{d_{2}}, \ldots, u_{d_{m}}\right] \in \Re^{m}, r(t) \in \Re^{m}$ is a vector of bounded reference input signals and $K \in \Re^{m \times n}$ is a constant gain vector such that the eigenvalues of $A-B K$ are set to the desired closed-loop system poles. The $v_{\eta}(t)=\left[v_{\eta_{1}}, v_{\eta_{2}}, \ldots, v_{\eta_{m}}\right] \in \Re^{m}$ term, is a commonly applied disturbance rejection term to compensate for the approximation error uncertainty and will be defined in the next section. The choice of $K$ can be made from a linear-quadratic regulator (LQR) design [35] or a pole placement technique [56].

Applying (6.12), (6.21) and (6.22), we obtain the closed-loop linear dynamics

$$
\begin{aligned}
\dot{x}(t)= & (A-B K) x(t)+\sum_{i=1}^{m} B_{i}\left(\eta_{i}(t)-\tilde{\theta}_{N_{i}}^{T}(t) \frac{\partial B_{N_{i}}\left(v_{i}(t), \alpha\right)}{\partial v_{i}(t)} \hat{\theta}_{s_{i}}^{T}(t) B_{s_{i}}\left(u_{d_{i}}(t), \alpha\right)\right. \\
& \left.+\hat{\theta}_{N_{i}}^{T}(t) \frac{\partial B_{N_{i}}\left(v_{i}(t), \alpha\right)}{\partial v_{i}(t)} \tilde{\theta}_{s_{i}}^{T}(t) B_{s_{i}}\left(u_{d_{i}}(t), \alpha\right)\right)+B r(t)+B v_{\eta}(t) .
\end{aligned}
$$

This representation motivates us to choose the reference model system as

$$
\dot{x}_{m}(t)=(A-B K) x_{m}(t)+B r(t) .
$$

The control objective is to choose a feedback gain $K$ and adaptive laws for the $i^{\text {th }}$ parameters $\hat{\theta}_{N_{i}}(t)$ and $\hat{\theta}_{s_{i}}(t)$, such that all closed-loop system signals are bounded, and the state tracking errors $e(t)=x(t)-x_{m}(t)$ are as small as possible (due to the uncertainty of each $\eta_{i}(t)$ and the related approximation, $\lim _{t \rightarrow \infty}\left(x(t)-x_{m}(t)\right)=0$ may not be theoretically achievable). 


\subsubsection{Adaptive Laws}

In this subsection, we formulate adaptive laws to update the $i^{\text {th }}$ parameter estimates $\hat{\theta}_{N_{i}}(t)$, $\hat{\theta}_{s_{i}}(t)$ and $\hat{\beta}_{i}(t)$, so that the control objective is achievable. Parameter projection is applied to ensure that the parameter estimates remain in the bounded region. We assume that the following inequalities for the components of the $i^{\text {th }}$ true nonlinearity parameters $\theta_{N_{i}}^{*}, \theta_{s_{i}}^{*}$ and $\beta_{i}$ are known and satisfy:

$$
\begin{gathered}
\theta_{1 N_{i}}^{b} \leq \theta_{1 N_{i}}^{*} \leq \theta_{1 N_{i}}^{d}, \ldots, \theta_{n_{1} N_{i}}^{b} \leq \theta_{n_{1} N_{i}}^{*} \leq \theta_{n_{1} N_{i}}^{d} \\
\theta_{1 s_{i}}^{b} \leq \theta_{1 s_{i}}^{*} \leq \theta_{1 s_{i}}^{d}, \ldots, \theta_{n_{2} s_{i}}^{b} \leq \theta_{n_{2} s_{i}}^{*} \leq \theta_{n_{2} s_{i}}^{d} \\
\beta_{1_{i}}^{b} \leq \beta_{1_{i}} \leq \beta_{1_{i}}^{d}, \ldots, \beta_{4_{i}}^{b} \leq \beta_{4_{i}} \leq \beta_{4_{i}}^{d}
\end{gathered}
$$

for some known constants $\theta_{1 N_{i}}^{b}, \ldots, \theta_{n_{1} N_{i}}^{b}, \theta_{1 N_{i}}^{d}, \ldots, \theta_{n_{1} N_{i}}^{d}$, where $n_{1}=M_{1} \cdot M_{2}$, and $\theta_{1 s_{i}}^{b}, \ldots, \theta_{n_{2} s_{i}}^{b}$, $\theta_{1 s_{i}}^{d}, \ldots, \theta_{n_{2} s_{i}}^{d}, n_{2}=R_{1} \cdot R_{2}, \beta_{1_{i}}^{b}, \ldots, \beta_{4_{i}}^{b}$, and $\beta_{1_{i}}^{d}, \ldots, \beta_{4_{i}}^{d}$. With these defines the $i^{\text {th }}$ initial estimates $\hat{\theta}_{N_{i}}(0), \hat{\theta}_{s_{i}}(0)$ and $\hat{\beta}_{i}(0)$ of $\theta_{N_{i}}^{*}, \theta_{s_{i}}^{*}$ and $\beta_{i}$ respectively, are chosen to satisfy the inequalities

$$
\begin{gathered}
\theta_{1 N_{i}}^{b} \leq \hat{\theta}_{1 N_{i}}(0) \leq \theta_{1 N_{i}}^{d}, \ldots, \theta_{n_{1} N_{i}}^{b} \leq \hat{\theta}_{n_{1} N_{i}}(0) \leq \theta_{n_{1} N_{i}}^{d}, \\
\theta_{1 s_{i}}^{b} \leq \hat{\theta}_{1 s_{i}}(0) \leq \theta_{1 s_{i}}^{d}, \ldots, \theta_{n_{2} s_{i}}^{b} \leq \hat{\theta}_{n_{2} s_{i}}(0) \leq \theta_{n_{2} s_{i}}^{d} \\
\beta_{1_{i}}^{b} \leq \hat{\beta}_{1_{i}}(0) \leq \beta_{1_{i}}^{d}, \ldots, \beta_{4_{i}}^{b} \leq \hat{\beta}_{4_{i}}(0) \leq \beta_{4_{i}}^{d} .
\end{gathered}
$$

Applying the parameter projection algorithm from [76], we develop our adaptive laws as

$$
\begin{gathered}
\dot{\hat{\theta}}_{N_{i}}(t)=g_{N_{i}}(t)+h_{N_{i}}(t), \\
\dot{\hat{\theta}}_{s_{i}}(t)=g_{s_{i}}(t)+h_{s_{i}}(t), \\
\dot{\hat{\beta}}_{i}(t)=g_{\beta_{i}}(t)+h_{\beta_{i}}(t),
\end{gathered}
$$

where $i=1,2, \ldots, m, t \geq 0, g_{N_{i}}(t), g_{s_{i}}(t)$ and $g_{\beta_{i}}(t)$ are the adaptation functions given by

$$
\begin{gathered}
g_{N_{i}}(t)=-\Gamma_{1_{i}} e^{T}(t) P B_{i} \frac{\partial B_{N_{i}}\left(v_{i}, \alpha\right)}{\partial v_{i}} \hat{\theta}_{s_{i}}^{T} B_{s_{i}}\left(u_{d_{i}}, \alpha\right) \\
g_{s_{i}}(t)=\Gamma_{2_{i}} e^{T}(t) P B_{i} \frac{\partial B_{N_{i}}\left(v_{i}, \alpha\right)}{\partial v_{i}} \hat{\theta}_{N_{i}}^{T} B_{s_{i}}\left(u_{d_{i}}, \alpha\right) \\
g_{\beta_{i}}(t)=\Gamma_{3_{i}} \Omega_{i}\left|e^{T}(t) P B_{i}\right|
\end{gathered}
$$


and $\Gamma_{j_{i}}, j=1,2,3$ are the adaptation gain matrices that satisfy $\Gamma_{j_{i}}=\Gamma_{j_{i}}^{T}>0$ and recall the state tracking errors from

$$
e(t)=x(t)-x_{m}(t)
$$

$P \in \Re^{n \times n}, P=P^{T}>0$ is determined by the solution to the Lyapunov equation for continuous time systems

$$
P A_{m}+A_{m}^{T} P=-Q
$$

for a constant matrix $Q \in \Re^{n \times n}, Q=Q^{T}>0$ (recall $A_{m}=A-B K$ ). The functions $h_{N_{i}}(t), h_{s_{i}}(t)$ and $h_{\beta_{i}}(t)$ are parameter projection functions defined such that the $i^{t h}$ parameter estimates stay in a convex region for certain desired physical properties, and are represented as

$$
\begin{aligned}
& h_{l N_{i}}(t)= \begin{cases}0 & \text { if } \hat{\theta}_{l N_{i}}(t) \in\left(\theta_{l N_{i}}^{b}, \theta_{l N_{i}}^{d}\right), \text { or } \\
& \text { if } \hat{\theta}_{l N_{i}}(t)=\theta_{l N_{i}}^{b}, g_{l N_{i}}(t) \geq 0, \text { or } \\
& \text { if } \hat{\theta}_{l N_{i}}(t)=\theta_{l N_{i}}^{d}, g_{l N_{i}}(t) \leq 0, \\
-g_{l N_{i}}(t) & \text { otherwise, }\end{cases} \\
& h_{j s_{i}}(t)= \begin{cases}0 & \text { if } \hat{\theta}_{j s_{i}}(t) \in\left(\theta_{j s_{i}}^{b}, \theta_{j s_{i}}^{d}\right), \text { or } \\
& \text { if } \hat{\theta}_{j s_{i}}(t)=\theta_{j s_{i}}^{b}, g_{j s_{i}}(t) \geq 0, \text { or } \\
& \text { if } \hat{\theta}_{j s_{i}}(t)=\theta_{j s_{i}}^{d}, g_{j s_{i}}(t) \leq 0, \\
-g_{j s_{i}}(t) & \text { otherwise, }\end{cases}
\end{aligned}
$$

where $i=1,2, \ldots, m, l=1,2, \ldots, M_{1} \cdot M_{2}$ and $j=1,2, \ldots, R_{1} \cdot R_{2}$, and

$$
h_{k \beta_{i}}(t)= \begin{cases}0 & \text { if } \hat{\beta}_{k_{i}}(t) \in\left(\beta_{k_{i}}^{b}, \beta_{k_{i}}^{d}\right), \text { or } \\ & \text { if } \hat{\beta}_{k_{i}}(t)=\beta_{k_{i}}^{b}, g_{k \beta_{i}}(t) \geq 0, \text { or } \\ & \text { if } \hat{\beta}_{k_{i}}(t)=\beta_{k_{i}}^{d}, g_{k \beta_{i}}(t) \leq 0, \\ -g_{k \beta_{i}}(t) & \text { otherwise, }\end{cases}
$$

where $k=1, \ldots, 4$. Note the coupled nature of the adaptive laws $\hat{\theta}_{N_{i}}(t)$ and $\hat{\theta}_{s_{i}}(t)$ clearly showing the mutual dependence of the two nonlinearity spline function approximators. Next, to determine the effectiveness of our design we analyze the stability properties.

Theorem 1 Under the facts stated in Lemma 2, the modified desired feedback control law (6.22), adaptive laws (6.26), (6.27) and (6.28), applied to the plant (6.21), guarantee that 
the overall closed-loop system is locally stable in the sense that the initial system states $x(0)$ are chosen from the desired compact set of operation $\mathcal{D}_{x} \subset \Re^{n}$, and the $i^{\text {th }}$ initial parameters $\left\{\hat{\theta}_{N_{i}}(0), \hat{\theta}_{s_{i}}(0)\right\} \in \mathcal{D}_{\theta_{i}}$, where $\mathcal{D}_{\theta_{i}} \subset \Re^{M_{1} \cdot M_{2}+R_{1} \cdot R_{2}}$ is a compact set in the space of $\theta_{N_{i}}^{*}, \theta_{s_{i}}^{*}$, then all signals remain bounded and the tracking error $e(t)$ is bounded by the approximation errors in a mean square sense.

Proof: Applying (6.23) and (6.24) we have

$$
\begin{aligned}
\dot{e}(t)= & (A-B K) e(t)+\sum_{i=1}^{m} B_{i}\left(v_{\eta_{i}}(t)+\eta_{i}(t)-\tilde{\theta}_{N_{i}}^{T}(t) \frac{\partial B_{N_{i}}\left(v_{i}(t), \alpha\right)}{\partial v_{i}(t)} \hat{\theta}_{s_{i}}^{T}(t) B_{s_{i}}\left(u_{d_{i}}(t), \alpha\right)\right. \\
& \left.+\hat{\theta}_{N_{i}}^{T}(t) \frac{\partial B_{N_{i}}\left(v_{i}(t), \alpha\right)}{\partial v_{i}(t)} \tilde{\theta}_{s_{i}}^{T}(t) B_{s_{i}}\left(u_{d_{i}}(t), \alpha\right)\right) .
\end{aligned}
$$

Consider the positive definite function

$$
V\left(e, \tilde{\theta}_{N_{i}}, \tilde{\theta}_{s_{i}}\right)=e^{T} P e+\sum_{i=1}^{m}\left(\tilde{\theta}_{N_{i}}^{T} \Gamma_{1_{i}}^{-1} \tilde{\theta}_{N_{i}}+\tilde{\theta}_{s_{i}}^{T} \Gamma_{2_{i}}^{-1} \tilde{\theta}_{s_{i}}+\tilde{\beta}_{i}^{T} \Gamma_{3_{i}}^{-1} \tilde{\beta}_{i}\right)
$$

as a measure of the systems error functions $e(t), \tilde{\theta}_{N_{i}}(t)$ and $\tilde{\theta}_{s_{i}}(t)$, where $\tilde{\beta}_{i}(t)=\hat{\beta}_{i}(t)-\beta_{i}$. Using the fact that $\dot{\tilde{\theta}}_{N_{i}}(t)=\dot{\hat{\theta}}_{N_{i}}(t), \dot{\tilde{\theta}}_{s_{i}}(t)=\dot{\hat{\theta}}_{s_{i}}(t)$, and $\dot{\tilde{\beta}}_{i}(t)=\dot{\hat{\beta}}_{i}(t)$ we write the time derivative of $V$ as

$$
\dot{V}=e^{T}(t) P \dot{e}(t)+\dot{e}^{T}(t) P e(t)+2 \sum_{i=1}^{m}\left(\tilde{\theta}_{N_{i}}^{T}(t) \Gamma_{1_{i}}^{-1} \dot{\hat{\theta}}_{N_{i}}(t)+\tilde{\theta}_{s_{i}}^{T}(t) \Gamma_{2_{i}}^{-1} \dot{\hat{\theta}}_{s_{i}}(t)+\tilde{\beta}_{i}^{T}(t) \Gamma_{3_{i}}^{-1} \dot{\hat{\beta}}_{i}(t)\right) .
$$

Substituting (6.26), (6.27), (6.28) and (6.37) into (6.39), and with the cancellation of like terms, the time derivative of $V$ becomes

$$
\begin{aligned}
\dot{V}= & -e^{T}(t) Q e(t)+2 \sum_{i=1}^{m}\left(e^{T}(t) P B_{i} v_{\eta_{i}}(t)+e^{T}(t) P B_{i} \eta_{i}(t)+\tilde{\beta}_{i}^{T} \Omega_{i}\left|e^{T}(t) P B_{i}\right|+\right. \\
& \left.+\tilde{\theta}_{N_{i}}^{T}(t) \Gamma_{1_{i}}^{-1} h_{N_{i}}(t)+\tilde{\theta}_{s_{i}}^{T}(t) \Gamma_{2_{i}}^{-1} h_{s_{i}}(t)+\tilde{\beta}_{i}^{T}(t) \Gamma_{3_{i}}^{-1} h_{\beta_{i}}(t)\right) .
\end{aligned}
$$

With each $i^{\text {th }}$ initial parameter within known upper and lower bounds, and from the defined parameter projection functions $h_{N_{i}}(t), h_{s_{i}}(t)$, and $h_{\beta_{i}}(t)$ which keep the parameters in the desired bounds, it follows that (6.25) is satisfied, and each $i^{\text {th }}$

$$
\tilde{\theta}_{N_{i}}^{T} h_{N_{i}}(t) \leq 0, \quad \tilde{\theta}_{s_{i}}^{T} h_{s_{i}}(t) \leq 0, \quad \tilde{\beta}_{i}^{T} h_{\beta_{i}}(t) \leq 0
$$


that is, $\tilde{\theta}_{N_{i}}^{T}(t) \Gamma_{1_{i}}^{-1} h_{N_{i}}(t) \leq 0, \tilde{\theta}_{s_{i}}^{T}(t) \Gamma_{2_{i}}^{-1} h_{s_{i}}(t) \leq 0$, and $\tilde{\beta}_{i}^{T}(t) \Gamma_{3_{i}}^{-1} h_{\beta_{i}}(t) \leq 0$. From these properties, (6.40) reduces to

$$
\dot{V} \leq-e^{T}(t) Q e(t)+2 \sum_{i=1}^{m}\left(e^{T}(t) P B_{i} \eta_{i}(t)+\tilde{\beta}_{i}^{T} \Omega_{i}\left|e^{T}(t) P B_{i}\right|+e^{T}(t) P B_{i} v_{\eta_{i}}(t)\right) .
$$

To proceed with the analysis we first consider the case where we ignore the uncertainty compensation signal $v_{\eta_{i}}(t)$ part in (6.42). This corresponds to an adaptive control scheme that ensures desired closed-loop stability and tracking properties which, like those with other approximation based designs, are in a local and average sense due to approximation errors, that is, for approximation errors with some non-zero bounds and chosen initial conditions within the specified regions, all closed-loop system signals remain bounded and the tracking error $e(t)$ is bounded by the approximation errors in a mean square sense.

Since $Q=Q^{T}>0$ and each $\eta_{i}(t)$ is small (in some norm sense) and bounded on a compact region, we have, from (6.40) and (6.41), the boundedness of $e(t)$, from (6.24) that of $x_{m}(t)$ and, in turn, from $e(t)=x(t)-x_{m}(t)$, that of $x(t)$, from (6.22), that of $u_{d}(t)$ (without $v_{\eta}(t)$ ). Thus all closed-loop signals are bounded. Finally, from (6.26), (6.27), $(6.28),(6.40)$, and $(6.41), e(t)$, each $i^{t h} \dot{\hat{\theta}}_{N_{i}}(t)$, and $\dot{\hat{\theta}}_{s_{i}}(t)$ are all bounded by $\eta_{i}(t)$ in a mean square sense (e.g. $\int_{t_{1}}^{t_{2}} e^{2}(t) d t \leq \gamma_{0}+k_{0} \sum_{i=1}^{m} \int_{t_{1}}^{t_{2}} \eta_{i}^{2}(t) d t$ for some constants $\gamma_{0}, k_{0}>0$ ).

Next, we consider the case where the $v_{\eta_{i}}(t)$ term is included in (6.42). Following the Lyapunov redesign procedure presented in $[30], v_{\eta_{i}}(t)$ is a chosen term to cancel the destabilizing effect of $\eta_{i}(t)$ and $\tilde{\beta}_{i}^{T} \Omega_{i}$ on $\dot{V}$. With this definition, we need to choose $v_{\eta_{i}}(t)$ such that $\sum_{i=1}^{m}\left(e^{T}(t) P B_{i} \eta_{i}(t)+\tilde{\beta}_{i}^{T} \Omega_{i}\left|e^{T}(t) P B_{i}\right|+e^{T}(t) P B_{i} v_{\eta_{i}}(t)\right) \leq 0$. First we realize that the inequality (6.17) is satisfied with the absolute value $|\cdot|$, that is,

$$
\left|\eta_{i}(t)\right| \leq \beta_{i}^{T} \Omega_{i} .
$$

Thus we have

$$
\begin{aligned}
& \sum_{i=1}^{m}\left(e^{T}(t) P B_{i} \eta_{i}(t)+\tilde{\beta}_{i}^{T} \Omega_{i}\left|e^{T}(t) P B_{i}\right|+e^{T}(t) P B_{i} v_{\eta_{i}}(t)\right) \\
\leq & \sum_{i=1}^{m}\left(e^{T}(t) P B_{i} v_{\eta_{i}}(t)+\tilde{\beta}_{i}^{T} \Omega_{i}\left|e^{T}(t) P B_{i}\right|+\left|e^{T}(t) P B_{i}\right|\left|\eta_{i}(t)\right|\right), \\
\leq & \sum_{i=1}^{m}\left(e^{T}(t) P B_{i} v_{\eta_{i}}(t)+\left|e^{T}(t) P B_{i}\right| \tilde{\beta}_{i}^{T} \Omega_{i}+\left|e^{T}(t) P B_{i}\right| \beta_{i}^{T} \Omega_{i}\right),
\end{aligned}
$$




$$
\leq \sum_{i=1}^{m}\left(e^{T}(t) P B_{i} v_{\eta_{i}}(t)+\left|e^{T}(t) P B_{i}\right| \hat{\beta}_{i}^{T} \Omega_{i}\right) .
$$

Ideally, we choose each $i^{\text {th }}$ term as

$$
v_{\eta_{i}}=-\epsilon_{i}(t) \operatorname{sgn}\left(e^{T}(t) P B_{i}\right),
$$

where $\epsilon_{i}(t) \geq \hat{\beta}_{i}^{T} \Omega_{i}$, then,

$$
\begin{gathered}
\sum_{i=1}^{m}\left(e^{T}(t) P B_{i} \eta_{i}(t)+\tilde{\beta}_{i}^{T} \Omega_{i}\left|e^{T}(t) P B_{i}\right|+e^{T}(t) P B_{i} v_{\eta_{i}}(t)\right) \\
\leq \sum_{i=1}^{m}\left(\hat{\beta}_{i}^{T} \Omega_{i}\left|e^{T}(t) P B_{i}\right|-\epsilon_{i}\left|e^{T}(t) P B_{i}\right|\right), \\
\leq \sum_{i=1}^{m}\left(\hat{\beta}_{i}^{T} \Omega_{i}\left|e^{T}(t) P B_{i}\right|-\hat{\beta}_{i}^{T} \Omega_{i}\left|e^{T}(t) P B_{i}\right|\right)=0 .
\end{gathered}
$$

Hence, with each $i^{\text {th }}$ approximation error rejection term $v_{\eta_{i}}(t)$ included, the $\dot{V}$ along the trajectories of the closed-loop system is negative definite, improving the adaptive control scheme's desired closed-loop stability and tracking properties.

$\nabla$

The $i^{\text {th }}$ robustifying control signal given by (6.45) is a discontinuous function and practically its implementation is characterized by the phenomenon of chattering, where, due to imperfections in switching devices or computational delays, the control has fast switching fluctuations across the region of operation [30]. To overcome these problems, we will approximate the discontinuous robustifying control signal by a continuous one. Observing the structure of (6.45) we chose our continuous approximation function as

$$
v_{\eta_{i}}(t)=-\epsilon_{i}(t) \frac{e^{T}(t) P B_{i}}{\rho_{i}+\left|e^{T}(t) P B_{i}\right|}, \quad 0<\rho_{i}<<1,
$$

where $i=1,2, \ldots, m$, and $|\cdot|$ is the absolute value. The system stability properties corresponding to this chosen continuous approximation function will be the same as (6.45), as long as the choice of $\rho_{i}$ is not too small and $\epsilon_{i}(t) \geq \hat{\beta}_{i}^{T} \Omega_{i}$. Thus far, we have developed a spline function approximation based smooth adaptive inverse design for compensating multiple input actuator nonlinearities.

In the next section, we apply our design to a benchmark commercial aircraft flight dynamic system with multiple input synthetic jet actuators. The following numerical evaluation of such an adaptive control system is crucial in order to verify the desired system stability and tracking properties. 


\subsection{Aircraft Flight Control Performance}

In this section, we demonstrate our spline function network approximation-based smooth adaptive inverse compensation control design applied to a commercial transport linear aircraft flight dynamic model with multiple input actuator nonlinearities, to evaluate the adaptive control system performance. We present the details of the aircraft dynamic model, actuator nonlinearities, nonlinearity inverse design, feedback control law, and simulation steps.

\subsubsection{Linear Aircraft Dynamic Model}

In this study, we employ a multi-input linearized model of a commercial transport aircraft that is,

$$
\dot{x}(t)=A x(t)+B u(t),
$$

where the state vector is $x=\left[x_{1}, x_{2}, x_{3}, x_{4}, x_{5}\right]^{T}$ whose components are the angle of attack $\alpha$, pitch rate $q$, side-slip angle $\beta$, roll rate $p$, and yaw rate $r$, the control input vector is $u=\left[u_{1}, u_{2}, u_{3}\right]^{T}$ whose components are the elevator angle $\delta_{e}$, aileron angle $\delta_{a}$ and rudder angle $\delta_{r}$, respectively. The elevator angle $\delta_{e}$ is to be equivalently implemented through synthetic jet actuators, and $A \in R^{5 \times 5}$ and $B \in R^{5 \times 3}$ which are given by

$$
\begin{aligned}
A=\left[\begin{array}{rrrrr}
-0.5656 & 0.9730 & 0 & 0 & 0 \\
-0.8985 & -0.4755 & 0 & 0 & 0 \\
0 & 0 & -0.1178 & 0.0501 & -0.9881 \\
0 & 0 & -1.4828 & -1.0674 & 0.6121 \\
0 & 0 & 0.5364 & -0.0644 & -0.3057
\end{array}\right] \\
B=\left[\begin{array}{rcc}
-0.0009 & 0 & 0 \\
-0.0161 & 0 & 0 \\
0 & 0 & 0.0007 \\
0 & 0.0136 & 0.0063 \\
0 & 0.0003 & -0.0079
\end{array}\right]
\end{aligned}
$$




\subsubsection{Linear Feedback Law}

Recall the desired linear feedback control law for the case when $u(t)=u_{d}(t)$ (that is, when there is no actuator nonlinearity $N(\cdot)$ and there is no inverse $\widehat{N I}(\cdot))$ is

$$
u_{d}(t)=-K x(t)+r(t)
$$

where $r(t)$ is a reference input signal, and $K \in R^{3 \times 5}$ is a feedback gain vector to be determined. To proceed, we augment the desired linear feedback control law with the $v_{\eta}(t)$ term to compensate for the approximation error uncertainty, such that

$$
u_{d}(t)=-K x(t)+r(t)+v_{\eta}(t)
$$

Using the LQR design, with simple $Q=I_{5}$ and $R=I_{3}$, we determine the optimal gain matrix for $K$ as

$$
K=\left[\begin{array}{rrrrr}
-0.0005 & -0.0163 & 0 & 0 & 0 \\
0 & 0 & -0.0046 & 0.0061 & 0.0033 \\
0 & 0 & 0.0090 & 0.0016 & -0.0434
\end{array}\right] .
$$

The reference model matrix $A_{m}=A-B K$ is

$$
A_{m}=\left[\begin{array}{rrrrr}
-0.5656 & 0.9730 & 0 & 0 & 0 \\
-0.8985 & -0.4758 & 0 & 0 & 0 \\
0 & 0 & -0.1178 & 0.0501 & -0.9881 \\
0 & 0 & -1.4828 & -1.0675 & 0.6123 \\
0 & 0 & 0.5365 & -0.0644 & -0.3060
\end{array}\right] .
$$

The closed-loop system poles are the eigenvalues of $A_{m}$ : $-0.5207+0.9339 i,-0.5207-$ $0.9339 i,-0.2179+0.7904 i,-0.2179-0.7904 i$ and -1.0555 . The solution to the Lyapunov equation $P A_{m}+A_{m}^{T} P=-Q=-I_{5}$, is

$$
P=\left[\begin{array}{rrrrr}
0.9728 & 0.0516 & 0 & 0 & 0 \\
0.0516 & 0.9535 & 0 & 0 & 0 \\
0 & 0 & 2.9439 & -3.0056 & 0.0026 \\
0 & 0 & -3.0056 & 4.3034 & -0.5925 \\
0 & 0 & 0.0026 & -0.5925 & 1.7631
\end{array}\right] .
$$




\subsubsection{Simulation}

To simulate the design, we need to first generate the signal $u_{d}(t)=-K x(t)+r(t)+v_{\eta}(t)$, for the reference signal $r(t)=3.7 \sin t$. The initial states for the system are:

$x_{0}=\left[\begin{array}{lllll}5 & 0.01 & 0.01 & 0.01 & 0.001\end{array}\right]^{T}$, and the reference system initial states are:

$x_{m 0}=\left[\begin{array}{lllll}4 & 0.06 & 0.05 & 0.08 & 0.009\end{array}\right]^{T}$. The plot in Figure 6.3, shows the system state tracking errors for the case when no nonlinearities are present in the control input actuators.

In Figure 6.2, all of the input channels contain a nonlinearity and as such the tracking errors are disturbed from zero.

The parameter estimates are initialized as $\hat{\theta}_{j, k}^{N}=1, \hat{\theta}_{j, k}^{s}=1$. With adaptation gains set to $\Gamma_{i}=300 \times I$, for $i=1,2,3$ and $I$ the identity matrix. The robustifying signal variable values from (6.47) are chosen as $\epsilon(t)=20$ and $\rho=0.00017$. The plot in Figure 6.3, shows the system state tracking errors for the case when no nonlinearities are present in the control input actuators. In Figure 6.4, one of the input channels contains a nonlinearity and as such some of the tracking errors are disturbed from zero. Figure 6.5 shows the state tracking errors with one input channel containing a nonlinearity and adaptive spline function compensation control, as we can see the tracking errors go near to zero. Figure 6.6 is a plot of the control signal. Figures 6.7-6.8 show the angle of attack output tracking performance. In Figure 6.9 we show the importance and effect of including the robustifying control signal $v_{\eta}(t)$ from (6.47). Figure 6.10 shows the regulation $(r(t)=0)$ results when one actuator ( $u_{1}$-the elevator angle) is implemented with synthetic jets. 


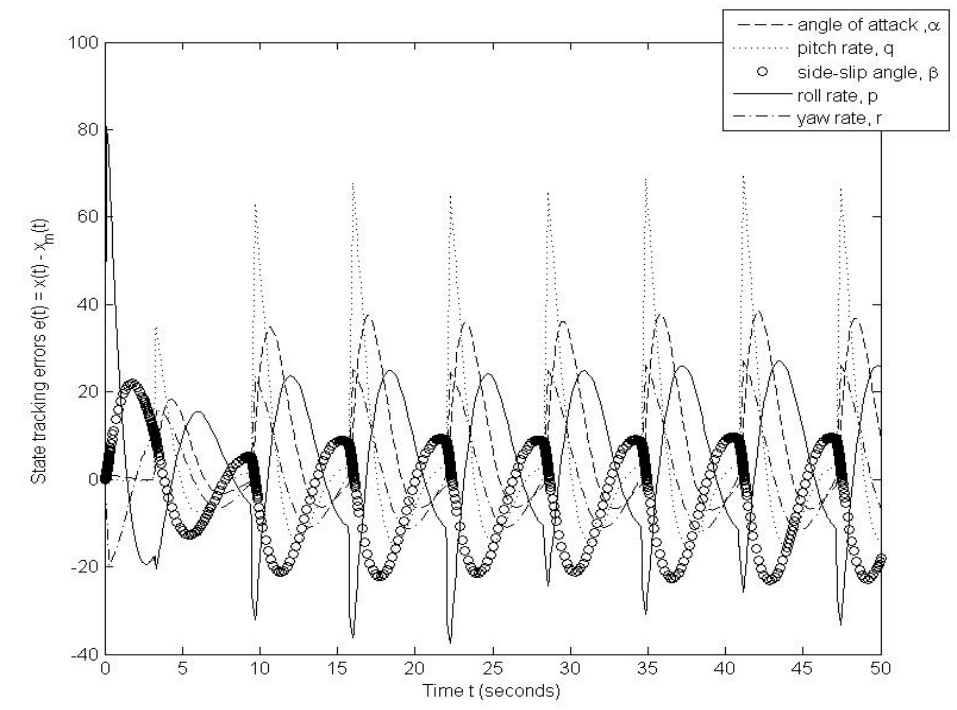

Figure 6.2: System state tracking errors with input nonlinearities.

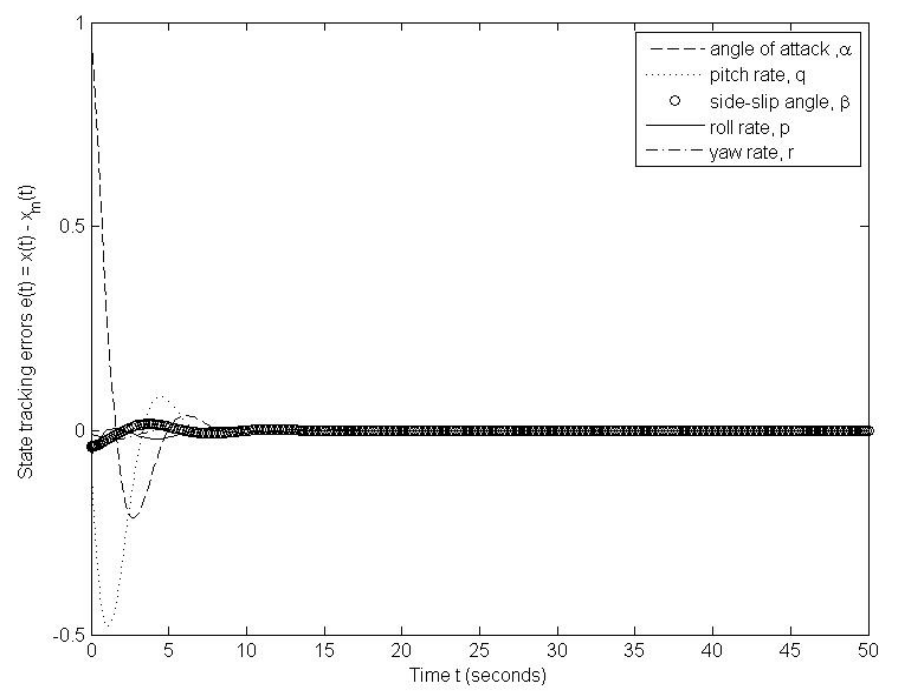

Figure 6.3: System state tracking errors without input nonlinearities. 


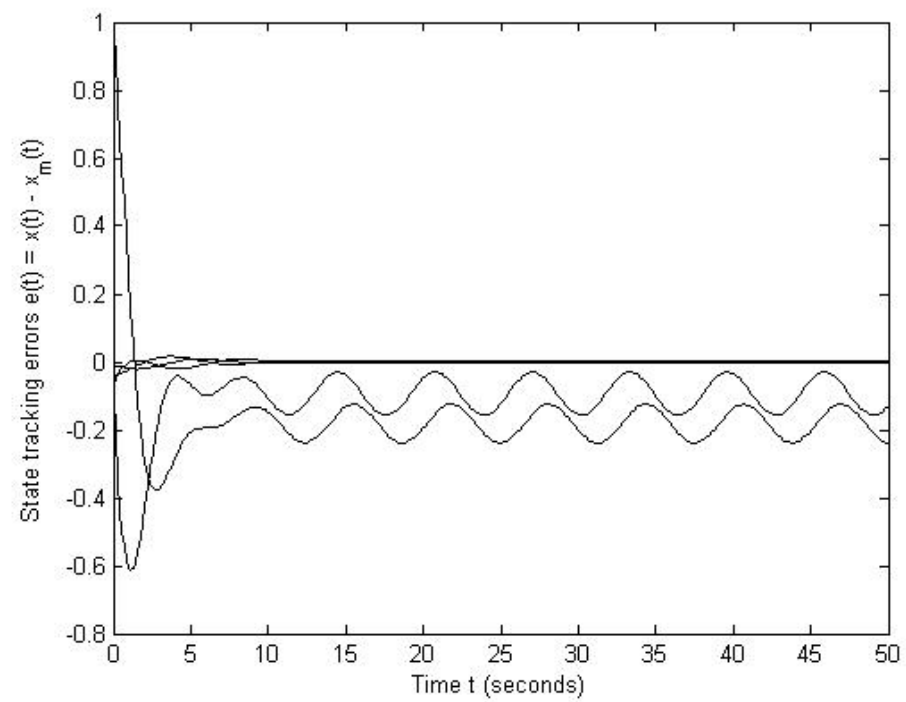

Figure 6.4: System state tracking errors with partial input nonlinearities.

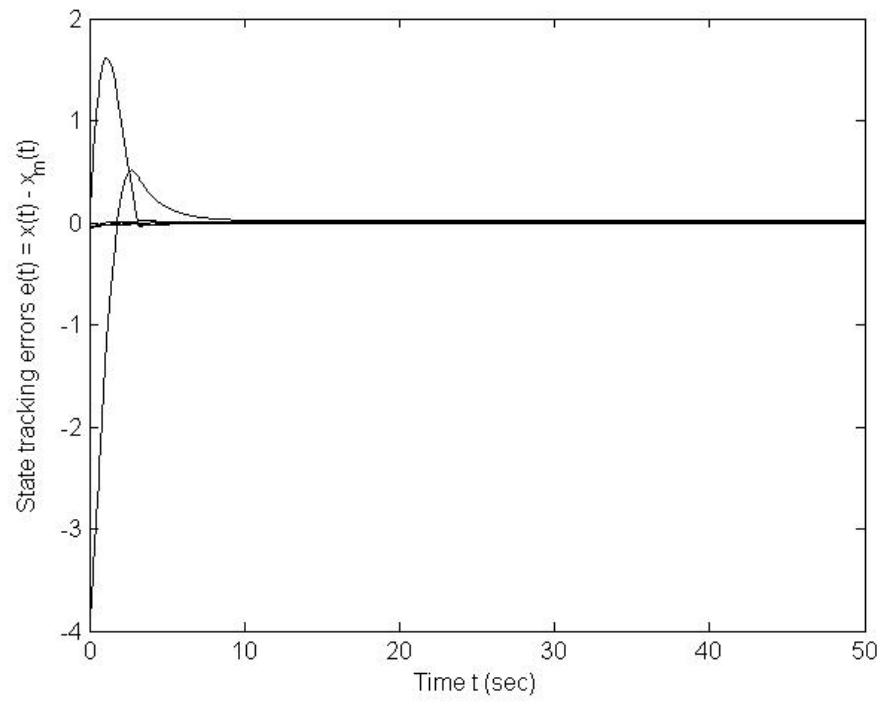

Figure 6.5: System state tracking errors with partial input nonlinearities and adaptive compensation. 


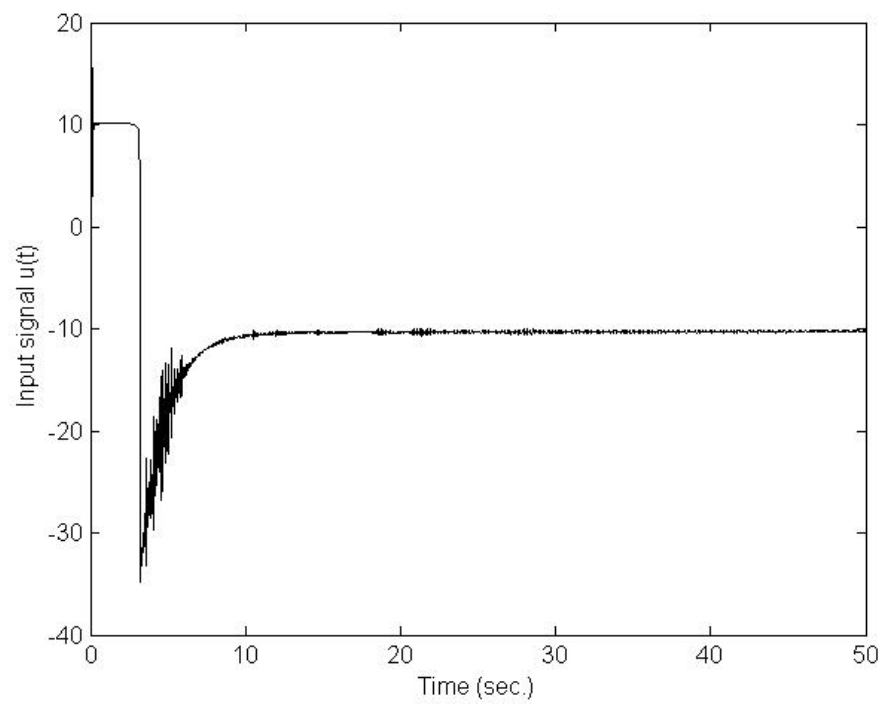

Figure 6.6: Control signal u(t).

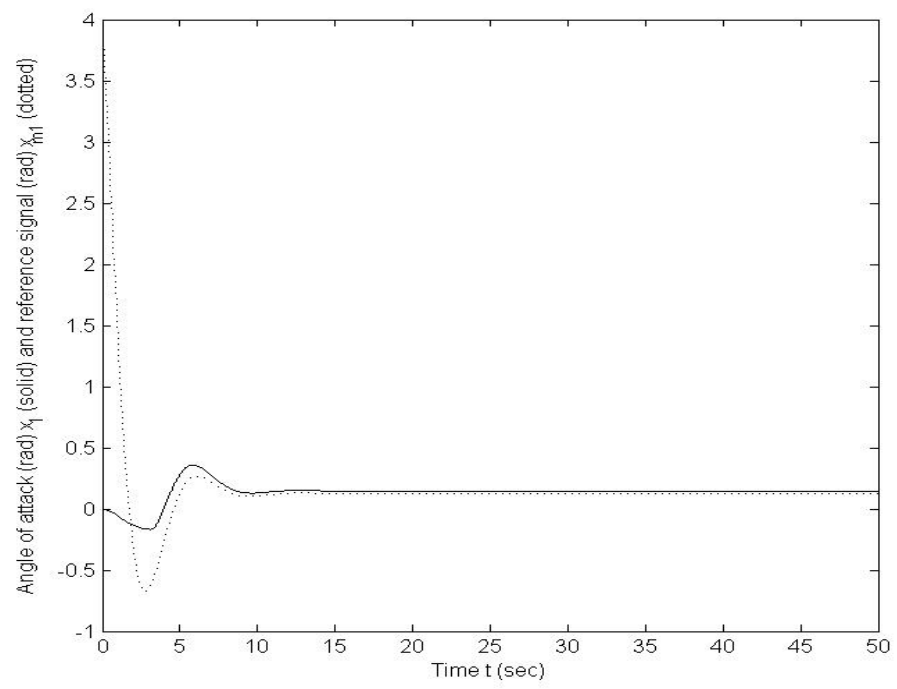

Figure 6.7: System output (solid) and reference signal (dotted): angle of attack (rad). 


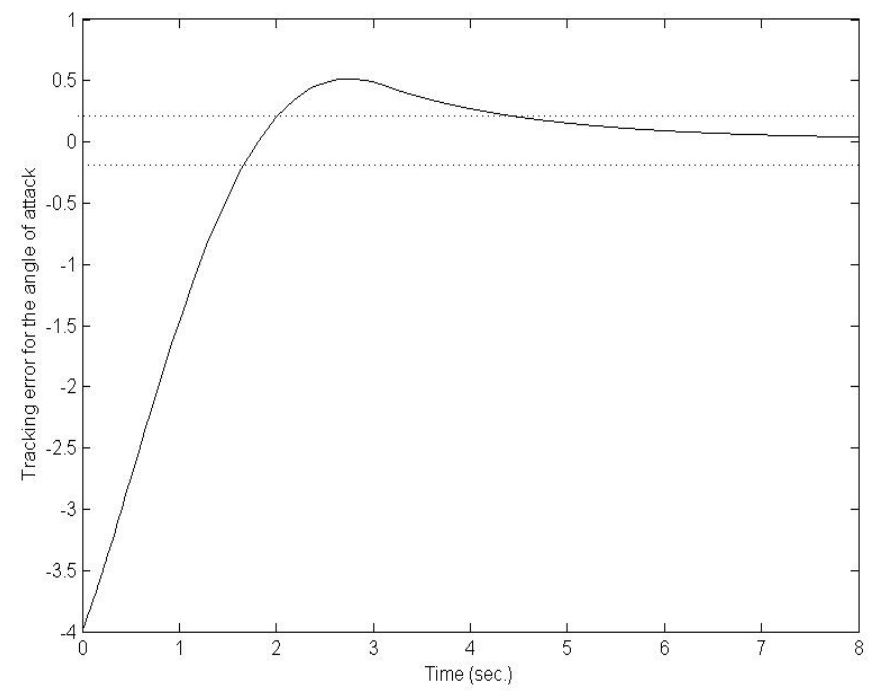

Figure 6.8: Tracking error during the first $8 \mathrm{~s}$ (the dashed lines indicate the required error bound).

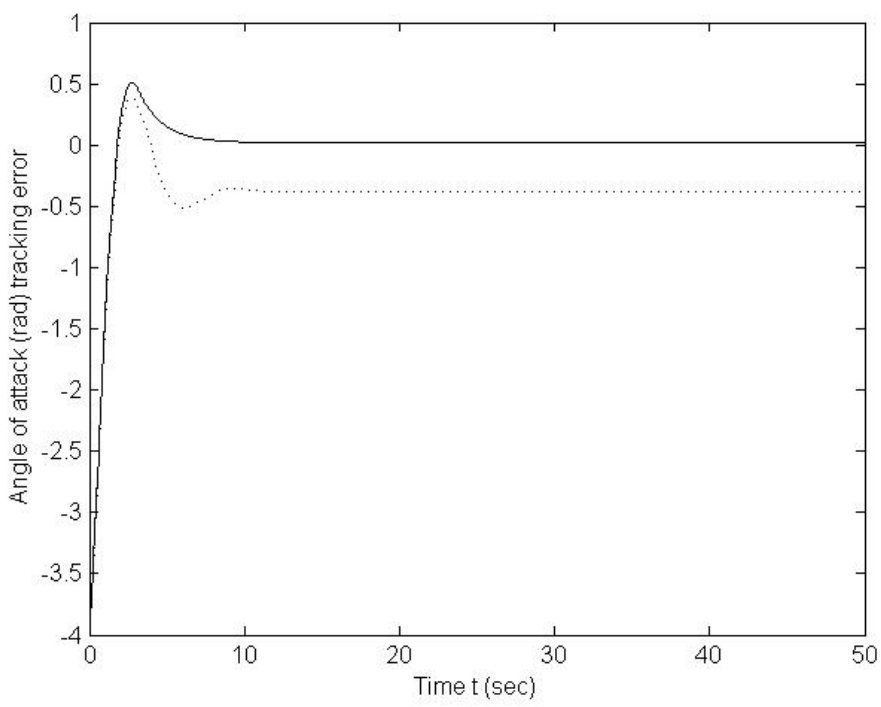

Figure 6.9: System output with robustifying signal (solid), without (dotted): angle of attack (rad). 

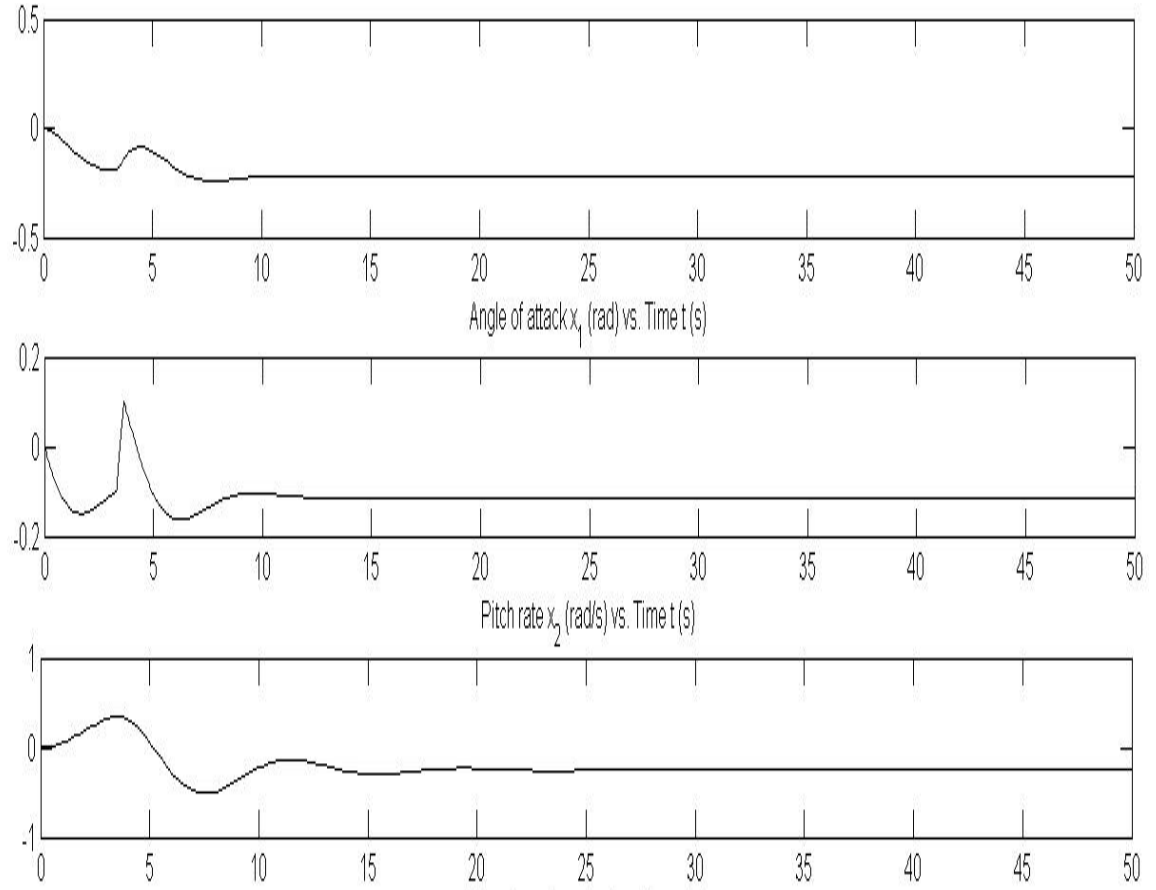

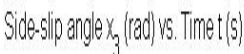
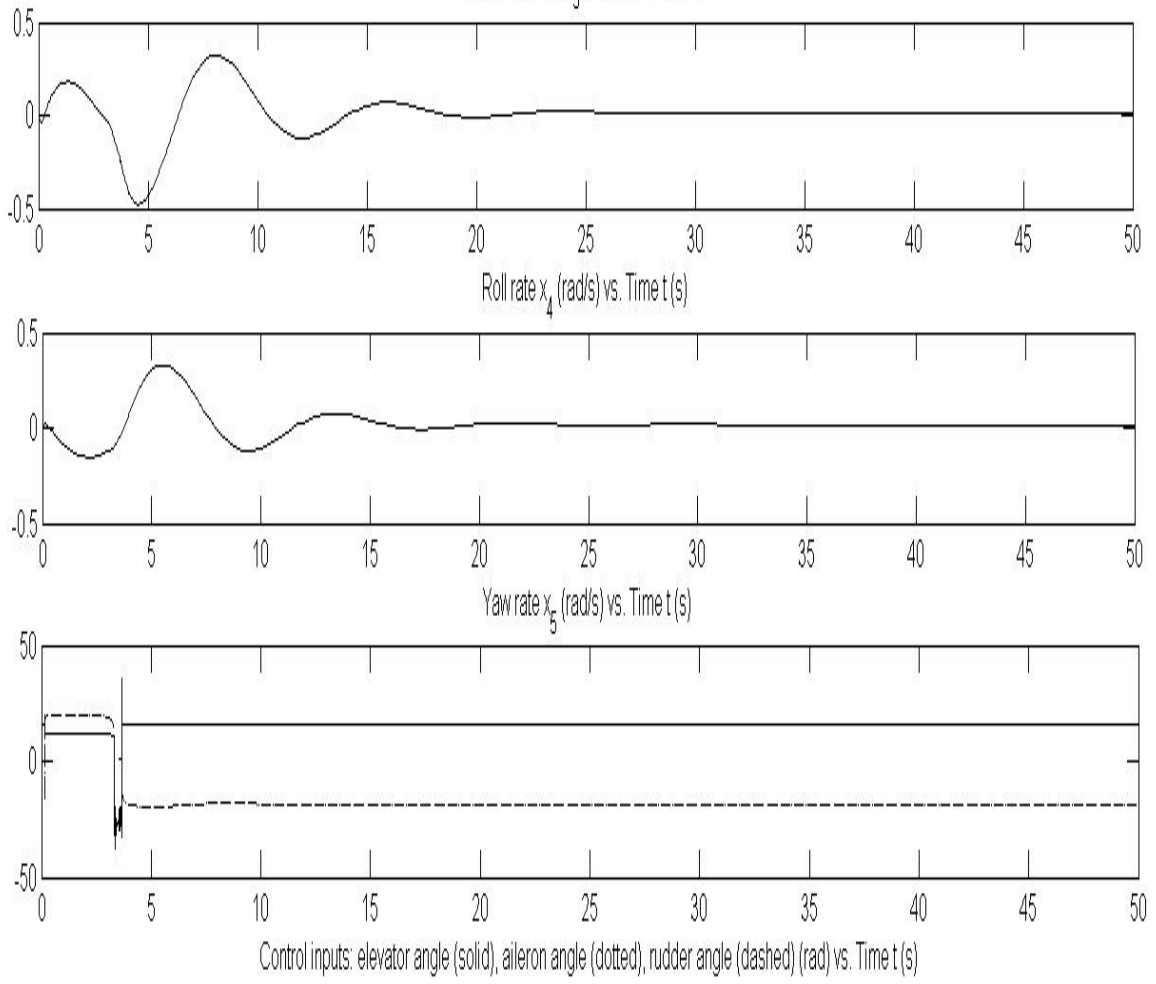

Figure 6.10: System response of regulation: $r(t)=0$. 


\section{Chapter 7}

\section{Conclusions and Outlook}

This chapter presents concluding remarks on our work presented in this dissertation. In addition, we present an outlook into future technology advancements. The purpose of this outlook is to inform the reader of current spacecraft technologies that are being pursued for further research. Fuel slosh in liquid fuel tanks of control systems are often poorly known and severely limit system performance. Liquid fuel tanks, a common system component, always contain fuel slosh.

\subsection{Conclusions}

The nonlinear effect of fuel slosh can be minimized by implementation of the spline function approximation based adaptive control scheme. Examples implementing both a SISO and MIMO spacecraft control model with fuel slosh are studied in this dissertation. This study demonstrated through simulation that fuel slosh in a partially filled spherical container deteriorated the attitude tracking for a sinusoidal reference. The simulation with the advanced adaptive controller showed a significant reduction in the effect of the unknown fuel slosh. Other common nonlinear effects such as thermally induced vibration on solar arrays and environmental disturbances can also be controlled using this adaptive control approach. The problem considered in this study is only one of many practical applications that can be studied for further advancements. Future work incorporating the multi-mass 
fuel slosh model with multiple inputs would improve the accuracy of the developed model and yield more insight into the system effects of liquid motion inside a container.

In addition, this dissertation document develops a method to use multivariable B-splines to construct two approximation structures for an adaptive inverse compensation controller for aircraft flight control. The first approximator estimates the unknown synthetic jet actuator nonlinearity, while the other estimates the inverse function for compensation. The use of spline functions for implementing these functions leads to a linear parametrization of the control error. A state feedback control law, combined with the adaptive inverse compensation scheme, has been designed and analyzed for desired stability and tracking performance, and applied to a realistic aircraft control example. Simulations are performed to illustrate the effectiveness of the design method developed.

\subsection{Technology Outlook}

There are a number of applications that are currently being pursued in spacecraft and aircraft technology. One of the more shocking but understandable is that spacecraft technology is soon to return to its roots with the return of the capsule spacecraft. The space shuttle design has been retired in 2010 [86], making room for a plethora of new innovative spacecraft designs. In addition to the damage that occurs to the spacecraft during the launch phase because of the rockets sending debris onto the attached spacecraft, which was concluded to be a possible culprit in the tragic Space Shuttle Columbia, that broke apart during the re-entry stage [86]. Implementing the capsule design for the spacecraft to be placed on top of the rockets for take off and do not run the risk of being damaged by rocket debris during the launch stage of the spacecraft mission.

On a side-note with satellite technology, currently the price of retrieving a damaged satellite and repairing it on earth and then sending it back to its original orbit is more than sending a new satellite. The average price for one of the state-of-the-art satellites can run in the hundreds of millions of dollars. This has inspired Boeing (the leading satellite provider) to pursue other technologies for their repairing needs. Some of the recent projects are sending satellites to repair other satellites while they remain in orbit. This application will 
be affected by fuel slosh because the amount of precision needed to complete the mission.

Commercial aircraft companies could see a substantial improvement in turbulence management systems by adding on synthetic jet control technologies to pre-existing airplanes. Safety is another aspect that would benefit from a secondary control method to assist the pilots in case of power loss or damaged component failures. Military applications similar to stealth aircraft could apply these new synthetic jet actuator technologies to allow for highly complex maneuvers that demand an increase in angle of attack values up to $40^{\circ}$.

Another recently developing technology that shows promise for spacecraft ranging in the 100 grams to $50 \mathrm{~kg}$ mass are NEMS engines [83]. Nano-Electrical-Mechanical-System (NEMS) are an exciting new field that is making its way into R\&D labs and commercial products. NEMS are developed by using a combination of micromachining and standard integrated circuit technology. Some of the most common NEMS applications are accelerometers, gyros, and GPS receivers. For spacecraft technology NEMS rocket engines are developed. The size of the NEMS engine is comparable to a human hair, but has a thrust-to-weight ratio hundreds of times greater than the macro size satellites and space shuttles [79].

The most recent space development is the new space race, which started around 2004, the race is to commercialize space travel. Researchers are studying cost efficient space travel and possibly orbiting a hotel in space for tourists to visit. Currently there are a number of companies that provide space travel service for civilians. These developments make way for a new meaning to space travel. Allowing more practical orbital flights for research or touring purposes.

To complete the construction of the $\$ 100$ billion international space station a substantial number of spacewalks must be performed. Traveling at 17,500 $\mathrm{mph}$ and 220 miles above the earth's surface, astronaut stability is crucial to maintain during spacewalk task performance. Current research presents extensions for robotic arms to enable under craft repair and maintenance. Formation flying is a concept used to solve the need to have large structures in space. Because it is close to impossible to launch a large space structure. Researchers are proposing sending an array of smaller structures that can in some way structure themselves together to form a larger structure that will be used for beneficial objectives. 


\section{Appendix}

\section{MIMO State Variable Model}

To place the system in state variable form we introduce the following state-variables:

$$
x_{1}=v_{x}, \quad x_{2}=v_{z}, \quad x_{3}=\theta, \quad x_{4}=\dot{\theta}, \quad x_{5}=\psi, \quad x_{6}=\dot{\psi} .
$$

With the system state variables defined, we write our nonlinear equations in statevariable form, by plugging in each corresponding state for the chosen variable:

$$
\begin{aligned}
\dot{x} & =f(x)+g(x) u, \\
y & =h(x)
\end{aligned}
$$

where

$$
u=\left[\begin{array}{c}
F_{T} \\
M
\end{array}\right], \quad y=\left[\begin{array}{c}
v_{z} \\
\theta
\end{array}\right]
$$

The system is governed by the set of equations (3.23)-(3.26), in compact form:

$$
\left[\begin{array}{c}
\dot{v}_{x} \\
\dot{v}_{z} \\
\ddot{\theta} \\
\ddot{\psi}
\end{array}\right]=N^{-1} G_{x}+N^{-1} G_{u} u
$$

where

$$
\begin{aligned}
& G_{x}=\left[\begin{array}{c}
F_{L}-m b \dot{\theta}^{2}-\left(m+m_{f}\right) \dot{\theta} v_{z}-m_{f} a(\dot{\theta}+\dot{\psi})^{2} \cos \psi \\
\left(m+m_{f}\right) \dot{\theta} v_{x}+m_{f} a(\dot{\theta}+\dot{\psi})^{2} \sin \psi \\
m b \dot{\theta} v_{x} \\
-\epsilon \dot{\psi}-m_{f} a \dot{\theta} v_{z} \sin \psi+m_{f} a \dot{\theta} v_{x} \cos \psi
\end{array}\right], \quad G_{u}=\left[\begin{array}{cc}
0 & 0 \\
1 & 0 \\
b & 1 \\
0 & 0
\end{array}\right], \\
& N=\left[\begin{array}{cccc}
m+m_{f} & 0 & m_{f} a \sin \psi & m_{f} a \sin \psi \\
0 & m+m_{f} & m_{f} a \cos \psi+m b & m_{f} a \cos \psi \\
0 & m b & I+m b^{2} & 0 \\
m_{f} a \sin \psi & m_{f} a \cos \psi & I_{f}+m_{f} a^{2} & I_{f}+m_{f} a^{2}
\end{array}\right]
\end{aligned}
$$


Recall the formula to solve for the inverse of a square matrix:

$$
N^{-1}=\frac{1}{\operatorname{det}(N)} \operatorname{adj}(N) .
$$

To find the $\operatorname{adj}(N)$ we first find the cofactor of each element of $N$ and take the transpose of the cofactor matrix of $N$, this results

$$
\operatorname{adj}(N)=\left[\begin{array}{cccc}
N_{11} & N_{21} & N_{31} & N_{41} \\
N_{12} & N_{22} & N_{32} & N_{42} \\
N_{13} & N_{23} & N_{33} & N_{43} \\
N_{14} & N_{24} & N_{34} & N_{44}
\end{array}\right] .
$$

Through some rigorous math we obtain

$$
\begin{aligned}
& N_{11}=\left(m+m_{f}\right)\left(I+m b^{2}\right)\left(I_{f}+m_{f} a^{2}\right)-m b\left(m_{f} a \cos x_{5}+m b\right)\left(I_{f}+m_{f} a^{2}\right)+ \\
& +m_{f} a \cos x_{5}\left(m b\left(I_{f}+m_{f} a^{2}\right)-m_{f} a \cos x_{5}\left(I+m b^{2}\right)\right), \\
& N_{12}=m_{f}^{2} a^{2} \sin x_{5} \cos x_{5}\left(I+m b^{2}\right), \\
& N_{13}=-m_{f}^{2} m a b \sin x_{5} \cos x_{5}, \\
& N_{14}=m_{f} a \sin x_{5}\left(\left(m+m_{f}\right)\left(I+m b^{2}\right)-m b\left(m_{f} a \cos x_{5}+m b\right)\right), \\
& N_{21}=m_{f} a^{2} \sin x_{5} \cos x_{5}\left(I+m b^{2}\right), \\
& N_{22}=\left(m+m_{f}\right)\left(I+m b^{2}\right)\left(I_{f}+m_{f} a^{2}\right)-m_{f}^{2} a^{2} \sin ^{2} x_{5}\left(I+m b^{2}\right), \\
& N_{23}=m_{f}^{2} a^{2} m b \sin ^{2} x_{5}-\left(m^{2} b+m_{f} m b\right)\left(I_{f}+m_{f} a^{2}\right), \\
& N_{24}=\left(m^{2} b+m b m_{f}\right)\left(I_{f}+m_{f} a^{2}\right)-m_{f}^{2} a^{2} m b \sin ^{2} x_{5}, \\
& N_{31}=-m_{f}^{2} a^{2} \sin x_{5} \cos x_{5} m b, \\
& N_{32}=m_{f}^{2} a^{2} m b \sin ^{2} x_{5}-\left(m^{2} b+m_{f} m b\right)\left(I_{f}+m_{f} a^{2}\right), \\
& N_{33}=\left(m+m_{f}\right)^{2}\left(I_{f}+m_{f} a^{2}\right)-\left(m+m_{f}\right) m_{f}^{2} a^{2} \cos ^{2} x_{5}-\left(m+m_{f}\right) m_{f}^{2} a^{2} \sin ^{2} x_{5}, \\
& N_{34}=\left(m+m_{f}\right) m_{f}^{2} a^{2} \sin ^{2} x_{5}-\left(m+m_{f}\right)^{2}\left(I_{f}+m_{f} a^{2}\right)+ \\
& +\left(m+m_{f}\right)\left(m_{f}^{2} a^{2} \cos ^{2} x_{5}+m b m_{f} a \cos x_{5}\right),
\end{aligned}
$$




$$
\begin{aligned}
& N_{41}=m^{2} b^{2} m_{f} a \sin x_{5}-\left(I+m b^{2}\right)\left(m_{a} m_{f} \sin x_{5}+m_{f}^{2} a \sin x_{5}\right) \\
& N_{42}=-\left(I+m b^{2}\right)\left(m m_{f} a \cos x_{5}+m_{f}^{2} a \cos x_{5}\right) \\
& N_{43}=m^{2} b m_{f} a \cos x_{5}+m b m_{f}^{2} a \cos x_{5} \\
& N_{44}=\left(m+m_{f}\right)^{2}\left(I+m b^{2}\right)-\left(m^{2} b+m b m_{f}\right)\left(m_{f} a \cos x_{5}+m b\right) .
\end{aligned}
$$

This assists us in solving for the determinant of $N$

$$
D_{N}=\operatorname{det}(N)=\left(m+m_{f}\right) N_{11}+m_{f} a \sin x_{5} N_{41},
$$

for simplicity we introduce

$$
D n_{i, j}=\frac{N_{i, j}}{D_{N}}, i, j=1,2,3,4
$$

and rewrite $G_{x}, G_{u}$ as

$$
G_{x}=\left[\begin{array}{c}
G_{1 x}(x) \\
G_{2 x}(x) \\
G_{3 x}(x) \\
G_{4 x}(x)
\end{array}\right], \quad G_{u}=\left[\begin{array}{cc}
0 & 0 \\
G_{2 u 1} & 0 \\
G_{3 u 1} & G_{3 u 2} \\
0 & 0
\end{array}\right]
$$

with

$$
\begin{aligned}
G_{1 x}(x) & =F_{L}-m b x_{4}^{2}-\left(m+m_{f}\right) x_{4} x_{2}-m_{f} a\left(x_{4}+x_{6}\right)^{2} \cos x_{5}, \\
G_{2 x}(x) & =\left(m+m_{f}\right) x_{4} x_{1}+m_{f} a\left(x_{4}+x_{6}\right)^{2} \sin x_{5}, \\
G_{3 x}(x) & =m b x_{4} x_{1}, \\
G_{4 x}(x) & =-\epsilon x_{6}-m_{f} a x_{4} x_{2} \sin x_{5}+m_{f} a x_{4} x_{1} \cos x_{5} . \\
G_{2 u 1} & =1, \quad G_{3 u 1}=b, \quad G_{3 u 2}=1 .
\end{aligned}
$$

This enables us to write the nonlinear system in affine control form as

$$
\begin{aligned}
\dot{x} & =f(x)+g(x) u, \\
y & =h(x)
\end{aligned}
$$


where

$$
f(x)=\left[\begin{array}{c}
f_{1}(x) \\
f_{2}(x) \\
x_{4} \\
f_{4}(x) \\
x_{6} \\
f_{6}(x)
\end{array}\right], g(x)=\left[\begin{array}{cc}
g_{11}(x) & g_{12}(x) \\
g_{21}(x) & g_{22}(x) \\
0 & 0 \\
g_{41}(x) & g_{42}(x) \\
0 & 0 \\
g_{61}(x) & g_{62}(x)
\end{array}\right],
$$

with

$$
\begin{aligned}
& f_{1}(x)=G_{1 x}(x) D n_{1,1}+G_{2 x}(x) D n_{2,1}+G_{3 x}(x) D n_{3,1}+G_{4 x}(x) D n_{4,1}, \\
& f_{2}(x)=G_{1 x}(x) D n_{1,2}+G_{2 x}(x) D n_{2,2}+G_{3 x}(x) D n_{3,2}+G_{4 x}(x) D n_{4,2}, \\
& f_{4}(x)=G_{1 x}(x) D n_{1,3}+G_{2 x}(x) D n_{2,3}+G_{3 x}(x) D n_{3,3}+G_{4 x}(x) D n_{4,3}, \\
& f_{6}(x)=G_{1 x}(x) D n_{1,4}+G_{2 x}(x) D n_{2,4}+G_{3 x}(x) D n_{3,4}+G_{4 x}(x) D n_{4,4}, \\
& g_{11}(x)=G_{2 u 1} D n_{2,1}+G_{3 u 1} D n_{3,1}, \quad g_{12}(x)=G_{3 u 2} D n_{3,1}, \\
& g_{21}(x)=G_{2 u 1} D n_{2,2}+G_{3 u 1} D n_{3,2}, \quad g_{22}(x)=G_{3 u 2} D n_{3,2}, \\
& g_{41}(x)=G_{2 u 1} D n_{2,3}+G_{3 u 1} D n_{3,3}, \quad g_{42}(x)=G_{3 u 2} D n_{3,3}, \\
& g_{61}(x)=G_{2 u 1} D n_{2,4}+G_{3 u 1} D n_{3,4}, \quad g_{62}(x)=G_{3 u 2} D n_{3,4},
\end{aligned}
$$

and $h(x)=\left[0, x_{2}, x_{3}, 0,0,0\right]^{T}$ as the system output.

\section{Reduced Order MIMO Model}

To place the reduced order system in state-variable form we define the state variables:

$$
x_{1}=v_{z}, \quad x_{2}=\theta, \quad x_{3}=\dot{\theta}, \quad x_{4}=\psi, \quad x_{5}=\dot{\psi},
$$

and write out the nonlinear equations (3.31)-(3.33) in state-variable form:

$$
\begin{aligned}
\dot{x} & =f(x)+g(x) u, \\
y & =h(x)
\end{aligned}
$$

where

$$
u=\left[\begin{array}{c}
F_{T} \\
M
\end{array}\right], \quad y=\left[\begin{array}{c}
v_{z} \\
\theta
\end{array}\right] .
$$


In compact form:

$$
\left[\begin{array}{c}
\dot{v_{z}} \\
\ddot{\theta} \\
\ddot{\psi}
\end{array}\right]=N^{-1} G_{x}+N^{-1} G_{u} u
$$

where

$$
\begin{aligned}
& G_{x}=\left[\begin{array}{c}
\left(m+m_{f}\right) \dot{\theta} v_{x}(t)+m_{f} a(\dot{\theta}+\dot{\psi})^{2} \sin \psi \\
m b \dot{\theta} v_{x}(t) \\
-\epsilon \dot{\psi}-\frac{m_{f} a F_{L}}{m+m_{f}} \sin \psi+m_{f} a \dot{\theta} v_{x}(t) \cos \psi
\end{array}\right], \quad G_{u}=\left[\begin{array}{cc}
1 & 0 \\
b & 1 \\
0 & 0
\end{array}\right], \\
& N=\left[\begin{array}{ccc}
m+m_{f} & m_{f} a \cos \psi+m b & m_{f} a \cos \psi \\
m b & I+m b^{2} & 0 \\
m_{f} a \cos \psi & I_{f}+m_{f} a^{2} & I_{f}+m_{f} a^{2}
\end{array}\right] .
\end{aligned}
$$

Recall the formula to solve for the inverse of a square matrix:

$$
N^{-1}=\frac{1}{\operatorname{det}(N)} \operatorname{adj}(N) \text {. }
$$

To find the $\operatorname{adj}(N)$ we first find the cofactor of each element of $N$ and take the transpose of the cofactor matrix of $N$, this results

$$
\operatorname{adj}(N)=\left[\begin{array}{ccc}
N_{11} & N_{21} & N_{31} \\
N_{12} & N_{22} & N_{32} \\
N_{13} & N_{23} & N_{33}
\end{array}\right] .
$$

Through some rigorous math we obtain

$$
\begin{aligned}
& N_{11}=\left(I+m b^{2}\right)\left(I_{f}+m_{f} a^{2}\right), \\
& N_{12}=-m b\left(I_{f}+m_{f} a^{2}\right), \\
& N_{13}=m b\left(I_{f}+m_{f} a^{2}\right)-\left(I+m b^{2}\right) m_{f} a \cos x_{4}, \\
& N_{21}=-m b\left(I_{f}+m_{f} a^{2}\right), \\
& N_{22}=\left(m+m_{f}\right)\left(I_{f}+m_{f} a^{2}\right)-m_{f}^{2} a^{2} \cos ^{2} x_{4}, \\
& N_{23}=\left(m b+m_{f} a \cos x_{4}\right) m_{f} a \cos x_{4}-\left(I_{f}+m_{f} a^{2}\right)\left(m+m_{f}\right), \\
& N_{31}=-\left(I+m b^{2}\right) m_{f} a \cos x_{4}, \\
& N_{32}=m b m_{f} a \cos x_{4}, \\
& N_{33}=\left(I+m b^{2}\right)\left(m+m_{f}\right)-m b\left(m b+m_{f} a \cos x_{4}\right) .
\end{aligned}
$$


This assists us in solving for the determinant of $N$

$$
D_{N}=\operatorname{det}(N)=m_{f} a \cos x_{4} N_{13}+\left(I_{f}+m_{f} a^{2}\right) N_{33},
$$

for simplicity we introduce

$$
D n_{i, j}=\frac{N_{i, j}}{D_{N}}, i, j=1,2,3
$$

and rewrite $G_{x}, G_{u}$ as

$$
G_{x}=\left[\begin{array}{c}
G_{1 x}(x) \\
G_{2 x}(x) \\
G_{3 x}(x)
\end{array}\right], \quad G_{u}=\left[\begin{array}{cc}
G_{1 u 1} & 0 \\
G_{2 u 1} & G_{2 u 2} \\
0 & 0
\end{array}\right],
$$

with

$$
\begin{aligned}
G_{1 x}(x) & =\left(m+m_{f}\right) x_{3} v_{x}(t)+m_{f} a\left(x_{3}+x_{5}\right)^{2} \sin x_{4}, \\
G_{2 x}(x) & =m b x_{3} v_{x}(t), \\
G_{3 x}(x) & =-\epsilon x_{5}-\frac{m_{f} a F}{m+m_{f}} \sin x_{4}+m_{f} a x_{3} v_{x}(t) \cos x_{4}, \\
G_{1 u 1} & =1, \quad G_{2 u 1}=b, \quad G_{2 u 2}=1 .
\end{aligned}
$$

This enables us to write out the nonlinear system in affine control form as

$$
\begin{aligned}
\dot{x} & =f(x)+g(x) u, \\
y & =h(x)
\end{aligned}
$$

where

$$
f(x)=\left[\begin{array}{c}
f_{1}(x) \\
x_{3} \\
f_{2}(x) \\
x_{5} \\
f_{3}(x)
\end{array}\right], g(x)=\left[\begin{array}{cc}
g_{11}(x) & g_{12}(x) \\
0 & 0 \\
g_{21}(x) & g_{22}(x) \\
0 & 0 \\
g_{31}(x) & g_{32}(x)
\end{array}\right]
$$

with

$$
\begin{aligned}
& f_{1}(x)=G_{1 x}(x) D n_{1,1}+G_{2 x}(x) D n_{2,1}+G_{3 x}(x) D n_{3,1}, \\
& f_{2}(x)=G_{1 x}(x) D n_{1,2}+G_{2 x}(x) D n_{2,2}+G_{3 x}(x) D n_{3,2},
\end{aligned}
$$




$$
\begin{aligned}
& f_{3}(x)=G_{1 x}(x) D n_{1,3}+G_{2 x}(x) D n_{2,3}+G_{3 x}(x) D n_{3,3}, \\
& g_{11}(x)=G_{1 u 1} D n_{1,1}+G_{2 u 1} D n_{2,1}, g_{12}(x)=G_{2 u 2} D n_{2,1}, \\
& g_{21}(x)=G_{1 u 1} D n_{1,2}+G_{2 u 1} D n_{2,2}, g_{22}(x)=G_{2 u 2} D n_{2,2}, \\
& g_{31}(x)=G_{1 u 1} D n_{1,3}+G_{2 u 1} D n_{2,3}, g_{32}(x)=G_{2 u 2} D n_{2,3},
\end{aligned}
$$

and $h(x)=\left[x_{1}, x_{2}, 0,0,0\right]^{T}$ as the system output. 


\section{Bibliography}

[1] H. Norman Abramson, "The Dynamic Behavior of Liquids in Moving Containers," NASA, SP-106, 1966.

[2] J.M. Adler, M.S. Lee and J.D. Saugen, "Adaptive Control of Propellant Slosh for Launch Vehicles," SPIE Sensors and Sensor Integration, Vol. 1480, pp. 11-22, 1991.

[3] B. Agrawal, "Dynamic Characteristics of Liquid Motion in Partially Filled Tanks of a Spinning Spacecraft," Journal of Guidance, Control, and Dynamics, Vol. 16, No. 4, July-August 1993, pp. 636-640.

[4] J.H. Ahlberg et al, The Theory of Splines and Their Applications, Academic Press, 1967.

[5] J. Ahmed, V. T. Coppola, D. S. Bernstein, "Adaptive Asymptotic Tracking of Spacecraft Attitude Motion with Inertia Matrix Identification," Journal of Guidance, Control and Dynamics, Volume 21, Sept.-Oct. 1998, pp. 684-691.

[6] M. Amitay, D. Pitt, V. Kibens, D.E. Parekh, A. Glezer, "Control of Internal Flow Separation Using Synthetic Jet Actuators," AIAA, paper No. 2000-0903, 2000.

[7] R. H. Bishop, S. J. Paytner, J. W. Sunkel, "Adaptive Control of Space Station with Control Moment Gyros," IEEE Control Systems Magazine, Oct. 1992, pg 23-27.

[8] S.A. Bortoff, "Pseudolinearization of the Acrobot Using Spline Functions", Proceedings of the 1992 Conference on Decision and Control.

[9] A. E. Bryson Jr., Control of Spacecraft and Aircraft. Princeton University Press, 1994. 
[10] C.T. Chen, Linear System Theory and Design. Oxford University Press, 3 ed., 1999.

[11] S. Cho, N.H. McClamroch and M. Reyhanoglu, "Feedback Control of a Space Vehicle with Unactuated Fuel Slosh Dynamics," Proceedings of AIAA Guidance, Navigation and Control Conference, 2000, Denver, CO, AIAA 2000-4046, Vol.1, pp. 354-359.

[12] V. A. Chobotov, Spacecraft Attitude Dynamics and Control. Krieger Publishing, 1991.

[13] D. Deb, G. Tao, J. Burkholder and D. Smith, "An Adaptive Inverse Control Scheme for a Synthetic Jet Actuator Model," Proceedings of the 2005 American Control Conference, pp. 2646-2651,Portland, Oregon, 2005.

[14] D. Deb, G. Tao, J. Burkholder and D. Smith, "Modeling and Compensation of Synthetic Jet Actuators for Airfoil Flow Seperation Control," Proceedings of the 2006 AIAA Guidance, Navigation and Control Conference, Keystone, CO, 2006.

[15] D. Deb, G. Tao and J. Burkholder, "Adaptive Approximation-Based Compensation of Synthetic Jet Actuator Nonlinearities for Aircraft Control," Proceedings of the 2007 AIAA Guidance, Navigation and Control Conference, Hilton Head, SC, 2007.

[16] F. T. Dodge, "The New Dynamic Behavior of Liquids in Moving Containers," SwRI, 2000.

[17] L. Ehrenwald, M. Guelman, "Integrated Adaptive Cotrol of Space Manipulators," Journal of Guidance, Control and Dynamics, Volume 21, Jan.-Feb. 1998, pg 156-163.

[18] J.A. Farrell, J.Y. Choi, "Nonlinear Adaptive Control Using Networks of Piecewise Linear Approximators," IEEE Transactions on Neural Networks, vol. 11, no. 2, pp. 390-401, March 2000.

[19] J.A. Farrell, M.M. Polycarpou, Adaptive Approximation-Based Control: Unifying Neural, Fuzzy and Traditional Adaptive Approximation Approaches, Wiley Series, 2006.

[20] G. Franklin, J. Powell, A. Emami-Naeini, Feedback Control of Dynamic Systems. Prentice Hall, 4 ed., 2002. 
[21] A.T. Fuller, "The Early Development of Control Theory," J. Dyn. Syst. Meas. Control, vol. 98, pp. 109-118, 224-235, 1976.

[22] S. S. Ge, C. C. Hang, T. H. Lee and T. Zhang, Stable Adaptive Neural Network Control, Kluwer Academic, Boston, MA, 2001.

[23] J. Genevaux, and D. Lu, "On Sloshing in a Container with Moving Walls," Journal of Fluids and Structures, Vol. 14, 2000, pp. 275-278.

[24] G. C. Goodwin and K. S. Sin, Adaptive Filtering Prediction and Control, Prentice-Hall, Englewood Cliffs, NJ, 1984.

[25] E.W. Graham, The Forces Produced by Fuel Oscillations in a Rectangular Tank. Douglas Aircraft Co, SM-13748, 1951.

[26] R. A. Ibrahim, Liquid Sloshing Dynamics, Theory and Applications, Cambridge, 2005.

[27] P. A. Ioannou and J. Sun, Robust Adaptive Control, Prentice-Hall, Upper Saddle River, NJ, 1996.

[28] A. Isidori, Nonlinear Control Systems, Springer-Verlag, 1989.

[29] J. Junkins, M. R. Akella, "Nonlinear Adaptive Control of Spacecraft Maneuvers," Journal of Guidance, Control and Dynamics, Volume 20, Nov.-Dec. 1997, pg 11041110.

[30] H.K. Khalil, Nonlinear Systems. Prentice Hall, 2002.

[31] H. Kimura, "A Quarterly Survey of New Products, Systems, and Technology," Mitsubishi Electric ADVANCE, Vol. 86, June 1999.

[32] M. Krstić, I. Kanellakopoulos and P. V. Kokotović, Nonlinear and Adaptive Control Design, John Wiley \& Sons, New York, 1995.

[33] J. Kuang and A.Y. Leung, "Feedback for Attitude Control of Liquid-Filled Spacecraft," Journal of Guidance, Control, and Dynamics, Vol. 24, No. 1, January- February 2001, pp. $46-55$. 
[34] Y. D. Landau, R. Lozano and M. M'Saad, Adaptive Control, Springer, London, 1998.

[35] F. L. Lewis, Applied Optimal Control and Estimation, Prentice-Hall, Englewood Cliffs, NJ, 1992.

[36] F. L. Lewis and Y. H. Kim, "Neural Network Output Feedback Control of Robot Manipulators," IEEE Transactions on Robotics and Automation, vol. 15, no. 2, pp. 301-309, 1999.

[37] Z. Mahfooz, M. K. Ahmed and H. Guoping, "Conceptual Designing and Numerical Modeling of Micro Pulse Jet for Controlling Flow Separation," International Journal of Engineering (IJE), vol. 5, no. 1, pp. 41-61, 2011.

[38] O. Mayr, The Origins of Feedback Control. Cambridge, MA: MIT Press, 1970.

[39] A. C. Miller, Flow Control Via Synthetic Jet Actuation. Austin, TX: Texas A \& M Press, 2004.

[40] A. S. Morse, "Global Stability of Parameter Adaptive Control Systems," IEEE Transactions on Automatic Control, vol. AC-25, no. 6, pp. 433-439, 1980.

[41] K. Narendra, A. Annaswamy, Stable Adaptive Systems. Prentice Hall, 1989.

[42] K.S. Narendra, K. Parthasarathy, "Identification and Control of Dynamical Systems Using Neural Networks", IEEE Transaction on Neural Networks, vol. 1, pp.4-27, 1990.

[43] A. G. Parlos, J. W. Sunkel, "Adaptive Attitude Control and Momentum Management for Large-Angle Spacecraft Maneuvers," Journal of Guidance, Control and Dynamics, vol. 15, Jul.-Aug.1992, pg 1018-1027.

[44] S. Paytner, R. Bishop, "Adaptive Nonlinear Attitude Control and Momentum Management of Spacecraft," Journal of Guidance, Control and Dynamics, vol. 20, Sept.Oct.1997, pg 1025-1032.

[45] L. D. Peterson, E. F. Crawley and R. J. Hansman, "Nonlinear Fluid Slosh Coupled to the Dynamics of a Spacecraft," AIAA Journal, 27, pp. 1230-1240, 1989. 
[46] T. Poggio, F. Girosi, "Networks for Approximation and Learning," Proceedings of the IEEE, vol. 78, no. 9, pp. 1481-1497, 1990.

[47] M. M. Polycarpou, P.A. Ioannou, "Identification and Control of Nonlinear System Using Neural Network Models: Design and Stability Analysis," Technical Report no. 91-09-01, Dept. of Electrical Engineering Systems, University of Southern California, Sep. 1991.

[48] M. M. Polycarpou, "Stable Adaptive Neural Control Scheme for Nonlinear Systems," IEEE Transactions on Automatic Control, vol. 41, pp. 447-451, 1996.

[49] M. M. Polycarpou, M. J. Mears, "Stable Adaptive Tracking of Uncertain Systems Using Nonlinearly Parametrized On-Line Approximators," International Journal of Control, vol. 70, no. 3, 1998, pg 363-384.

[50] M. M. Polycarpou, M. J. Mears, "Stable Neural Control of Uncertain Multivariable Systems," International Journal of Adaptive Control and Signal Processing, vol. 17, pp. 447-466, 2003.

[51] M. Reyhanoglu, "Maneuvering Control Problems for a Spacecraft with Unactuated Fuel Slosh Dynamics," IEEE Proceedings of Conference on Control Applications, pp. 695-699, June 2003.

[52] M. Reyhanoglu, J.R. Hervas "Nonlinear Control of Space Vehicles with Multi-Mass Fuel Slosh Dynamics," IEEE Proceedings of Conference on Recent Advances in Space Technologies (RAST), pp. 247-252, June 2011.

[53] M. Reyhanoglu, J.R. Hervas "Nonlinear Control of a Spacecraft with Multiple Fuel Slosh Modes," IEEE Proceedings of Conference on Decision and Control and European Control Conference (CDC-ECC), pp. 6192-6197, December 2011.

[54] J.R. Rice, The Approximation of Functions, Vol. 1. Addison-Wesley, Reading, MA, 1964.

[55] T.J. Rivlin, An Introduction to Approximation of Functions. New York: Dover, 1969. 
[56] W.J. Rugh, Linear System Theory, 2nd ed., Prentice-Hall, Englewood Cliffs, NJ, 1996.

[57] R.M. Sanner, J-J.E. Slotine, "Gaussian Networks for Direct Adaptive Control," IEEE Transactions on Neural Networks, vol. 3, no. 6, pp. 837-863, 1992.

[58] A. Santhanakrishnan, J.D. Jacob, "Flow Control with Plasma Synthetic Jet Actuators," Journal of Physics D: Applied Physics, vol.40, pp. 637-651, 2007.

[59] S. Sastry and M. Bodson, Adaptive Control: Stability, Convergence, and Robustness, Prentice-Hall, Englewood Cliffs, NJ, 1989.

[60] G. Schilling, "Sloshing in Space," Scientific American, vol. 291, pp. 22-23, August 2004.

[61] I.J. Schoenberg, "Contribution to the Problem of Approximation of Equidistant Data by Analytic Functions," Applied Mathematics, vol. 4, pp. 45-88, August 1946.

[62] L.L. Schumaker, Spline Functions: Basic Theory. New York: Wiley, 1981.

[63] R.R. Selmic, F.L. Lewis, "Deadzone Compensation in Motion Control Systems Using Neural Networks," IEEE Transactions on Automatic Control, vol. 45, no.4, pp. 602613, April 2000.

[64] H. Shageer, G. Tao, "Modeling and Adaptive Control of Spacecraft with Fuel Slosh," AIAA Conference Proceedings of Guidance, Navigation, and Control, Hilton Head Island, SC, August 2007.

[65] H. Shageer, G. Tao, "Zero Dynamics Analysis for Spacecraft with Fuel Slosh," AIAA Conference Proceedings of Guidance, Navigation, and Control, Honolulu, HI, August 2008.

[66] H. Shageer, G. Tao, and J. Burkholder, "Adaptive Spline Function-Based Compensation of Synthetic Jet Actuators for Aircraft Flight Control," Proceedings of the 2008 Conference on Decision and Control, pp. 1937-1942, Dec 2008.

[67] M.J. Sidi, Spacecraft Dynamics and Control A Practical Engineering Approach. Cambridge Aerospace Series, 1997. 
[68] J.-J.E. Slotine and M.D. Di Benedetto, "Hamiltonian Adaptive Control of Spacecraft," IEEE Transaction on automatic Control, Vol. 35, No. 7, July 1990, pp. 848-852.

[69] J. E. Slotine, W. Li, Applied Nonlinear Control. Prentice-Hall: Englewood Cliffs, NJ, 1991.

[70] B.L. Smith and A. Glezer, "Vectoring of a High Aspect Ratio Rectangular Air Jet using a Zero-Net-Mass-Flux Control Jet," Bull. Am. Phys. Soc. 39, 1994.

[71] D. Smith, M. Amitay, V. Kibens, D. Parekh, and A. Glezer, "Modification of the Lifting Body Aerodynamics by Synthetic Jet Actuators," AIAA, paper No. 98-0209, 1998.

[72] J. T. Spooner, M. Maggiore, R. Ordsqez, and K. M. Passino, Stable Adaptive Control and Estimation for Nonlinear Systems: Neural and Fuzzy Approximator Techniques, John Wiley and Sons, New York, 2002.

[73] T.E. Strikwerda et al, "NEAR Shoemaker: Major Anomaly Survival, Delayed Rendezvous and Mission Success," Journal in Guidance and Control 2001, R.D. Culp and C.N. Schira, Eds. San Siego, CA: Univelt, 2001, pp. 597-614.

[74] W.I. Sullivan et al, "A New Satellite Attitude Control System," Princeton Satellite systems and CTA space systems, 1997.

[75] I. E. Sumner, "Experimentally Determined Pendulum Analogy of Liquid Sloshing in Spherical and Oblate-Spheroidal Tanks," NASA, TN D-2737, 1965.

[76] G. Tao, Adaptive Control Design and Analysis. John Wiley \& Sons, 2003.

[77] M. Unser, "Splines: A Perfect Fit for Signal and Image Processing," IEEE Signal Processing Magazine, vol.16, Issue 6, pp. 22-38, Nov. 1999.

[78] Jan P.B. Vreeburg, "Spacecraft Maneuvers and Slosh Control," IEEE Control Systems, pp. 12-16, June 2005.

[79] K. J. Walchko, 2003, "Robust Nonlinear Attitude Control with Disturbance Compensation," Ph.D. Dissertation, University of Florida. 
[80] H.L. Wei, S.A. Billings, "An Efficient Nonlinear Cardinal B-spline Model for High Tide Forecasts at the Venice Lagoon," Nonlinear Processes in Geophysics,13, 577-584, 2006.

[81] N. Wiener, Cybernetics. MIT Press, Boston, 1948.

[82] J.T.-Y. Wen and K. Kreutz-Delgado, "The Attitude Control Problem," IEEE transactions on automatic control, Vol. 36, No. 10, pp. 1148-1162, October 1991.

[83] J. Wilson, "Major New Thrust for MEMS Engines," Aerospace America, pp.34-38, February 2003.

[84] B. Wittenmark and K. J. Åström, Adaptive Control, 2nd ed., Addison-Wesley, Reading, MA, 1995.

[85] J. Zhou, M.J. Er, J.M. Zurada, "Adaptive Neural Network Control of Uncertain Nonlinear Systems with Nonsmooth Actuator Nonlinearities," Neurocomputing Letters 70, Elsevier (2007), 1062-1070, 2006.

[86] http://www.nasa.gov, retrieved within May 2005 - March 2013.

[87] http://www.nasaspaceflight.com, search retrieved within Jul 2007 - March 2013.

[88] http://www.nlr.nl, National Aerospace Laboratory (NLR) in the Netherlands, retrieved in Nov 2005.

[89] www.google.com, search retrieved within May 2005 - April 2013.

[90] http://www.m-w.com, search retrieved Feb. 2007 - April 2013.

[91] www.wikipedia.org, search retrieved Feb. 2007 - April 2013. 